\title{
Modelo multi-estados markoviano não homogêneo com efeitos dinâmicos
}

\section{Iracema Hiroko Iramina Arashiro}

\author{
TESE APRESENTADA \\ $\mathrm{AO}$ \\ Instituto DE MATEmÁtica E EstatísticA \\ DA \\ Universidade DE SÃo PAUlo \\ PARA \\ OBTENÇÃO DO TÍTULO \\ $\mathrm{DE}$ \\ Doutor EM CIÊnCIAS
}

Área de Concentração: Estatística

Orientador: Prof. Dr. Antonio Carlos Pedroso de Lima

Durante o desenvolvimento deste trabalho, a autora recebeu auxílio financeiro da

CAPES (março/2003 a junho/2006) e CNPq (julho/2006 a fevereiro/2007).

São Paulo, maio de 2008. 



\title{
Modelo multi-estados markoviano não homogêneo com efeitos dinâmicos
}

\author{
Este exemplar corresponde à redação \\ final da tese devidamente corrigida \\ e defendida por Iracema Hiroko Iramina Arashiro \\ e aprovada pela Comissão Julgadora.
}

Banca Examinadora:

- Prof. Dr. Antonio Carlos Pedroso de Lima (orientador) - IME/USP

- Prof. Dr. Julio Singer - IME/USP

- Profa. Dra. Dione Maria Valença - UFRN

- Prof. Dr. Francisco Louzada Neto - UFSCar

- Prof. Dr. Vicente Garibay Cancho - ICMC/USP 

Ao meu marido Renato; às minhas filhas Hitomi, Sayuri e Kaori; aos meus queridos sobrinhos: Yuri, Albert, Valéria, Vanessa, Morikazu, Jorginho, Hideo, Ayumi, Aline, Yukari, Emi, Miki (Iraceminha), Akio, Vivi, Karen, Yuiti e Erica; e aos meus pais Seiko e Fujiko Iramina. 



\section{Agradecimentos}

Agradeço, em primeiro lugar, a Deus por me dar forças, saúde e serenidade durante o doutorado;

Ao Antonio Carlos, pelo incentivo, encorajamento, apoio, orientação e colaboração;

À pesquisadora Ana Paula Restiffe, pela disponibilização dos dados e pelas discussões;

Ao meu marido Renato, que esteve pacientemente me apoiando nos momentos difíceis, não deixando que o desânimo tomasse conta. E pelas inúmeras vezes em que assumiu o papel de pai e mãe;

Às minhas filhas, pelos momentos de alegria e força. Vocês são meu orgulho, pois apesar da minha ausência sempre foram boas meninas. Nossa! Como vocês cresceram durante o meu doutorado!

Ao meu pai, Seiko Iramina, que me apoiou com seu modo silencioso, e à minha mãe Fujiko Iramina, que sofreu muito em seus últimos meses de vida, mas, enquanto esteve consciente, sempre incentivou meus estudos;

Aos professores do IME-USP, que ajudaram a enriquecer meus conhecimentos, especialmente ao professor Julio Singer pelas discussões e sugestões;

Aos meus amigos e familiares, que sempre me incentivaram e torceram por mim. Saibam que eu me orgulho muito de todos vocês! 
À minha amiga Gisela Tunes, pelas nossas conversas, pelo apoio e pelos conselhos sobre as minhas filhas adolescentes;

À Maria Kelly Venezuela, que sempre se prontificou em esclarecer minhas dúvidas estatísticas e não estatísticas;

À minha amiga Andréia Coutinho, minha companheira do fretado Dinâmica-ABC/USP, que me ajudou esclarecendo as dúvidas matemáticas e compartilhou as dificuldades desta caminhada. Será que vamos sentir saudades das longas horas de trânsito?

Aos amigos Michelli Karine Barros, Patricia Espinheira, Raydonaldo Ospina, Juvêncio dos Santos Nobre, Célia Lopes Mendes, Jacqueline Batista, Frederico Poleto, Sumaia Latif, Lourdes Montenegro, Patricia Hess, Angela Tavares Paes e tantos outros que estiveram presentes e torcendo por mim neste longo e difícil percurso. 


\section{Resumo}

Modelos multi-estados têm sido utilizados para descrever o comportamento de unidades amostrais cuja principal resposta é o tempo necessário para a ocorrência de seqüências de eventos. Consideramos um modelo multi-estados markoviano, não homogêneo, que incorpora covariáveis cujos efeitos podem variar ao longo do tempo (efeitos dinâmicos), o que permite a generalização dos modelos usualmente empregados. Resultados assintóticos mostram que procedimentos de estimação baseados no método histograma crivo convergem para um processo gaussiano. A metodologia proposta mostra-se adequada na modelagem de dados reais para comparação de desenvolvimento de recém-nascidos pré-termo com os a termo. Estudos com dados gerados artificialmente confirmam os resultados teóricos obtidos.

Palavras-chave: Coeficiente dependente do tempo, histograma crivo, modelos multiestados. 



\section{Abstract}

Multi-state models have been used to describe the behavior of sample units where the principal response is the time needed for the occurrence of a sequence of events. We consider a nonhomogeneous Markovian multi-state model that incorporates covariates with time-dependent coefficient (dynamic effects), generalizing models usually employed. The asymptotic results show that the estimators based on the method of histogram sieves converge to a Gaussian process. The proposed methodology revels adequated for modeling data related to the comparison of developement of preterm infants with term infants. The studies with artificially generated data confirm the asymptotic results.

Keywords: Histogram sieves, multi state models, time-dependent coefficient. 



\section{Índice}

\begin{tabular}{lll}
\hline & Introdução & 1
\end{tabular}

$\begin{array}{lll}2 & \text { Modelos multi-estados } & 5\end{array}$

2.1 Introdução . . . . . . . . . . . . . . . . . . . . . . . 5

2.2 Formulação do modelo . . . . . . . . . . . . . . . . . . . . . 7

2.3 Coeficientes dependentes do tempo . . . . . . . . . . . . . . . . . 15

2.4 Especificação do modelo . . . . . . . . . . . . . . . . . . . . . . . . 16

\begin{tabular}{llr}
\hline 3 & Teoria assintótica & 19
\end{tabular}

3.1 Verossimilhança . . . . . . . . . . . . . . . . . . . . . . . . . . . . . . 19

3.2 Propriedades assintóticas do estimador de crivo . . . . . . . . . . . . . . . . . 22

3.2.1 Suposições . . . . . . . . . . . . . . . . . . . . . . . . . . . . . . 23

3.2.2 Consistência . . . . . . . . . . . . . . . . . . . . . . . . . . . . . . 25

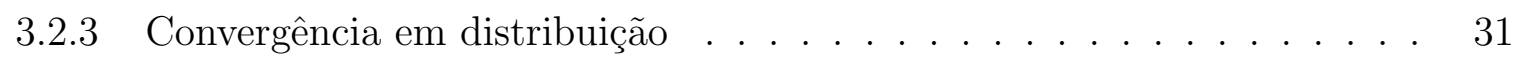

3.2 .4 Consistência do estimador da covariância . . . . . . . . . . . . . . . . . 40 
4 Aplicação a dados reais 45

4.1 Modelo markoviano homogêneo . . . . . . . . . . . . . . . . . . . . . . 46

4.2 Modelo markoviano não homogêneo . . . . . . . . . . . . . . . . . . . . . . . . 49

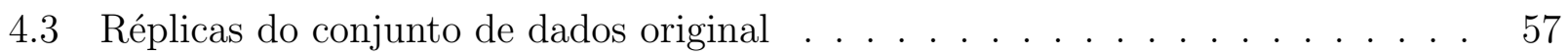

4.4 Censuras $\ldots \ldots \ldots \ldots$

$4.4 .1 \quad 15 \%$ de censuras $\ldots \ldots \ldots$. . . . . . . . . . . . . . . . . 60

$4.4 .2 \quad 30 \%$ de censuras $\ldots \ldots \ldots \ldots$. . . . . . . . . . . . . . . . . 61

$4.4 .3 \quad 50 \%$ de censuras $\ldots \ldots \ldots \ldots$. . . . . . . . . . . . . . . . . 62

\begin{tabular}{|lll}
\hline 5 & Considerações finais & 67
\end{tabular}

\begin{tabular}{|ll}
\hline A Lemas & 71
\end{tabular}

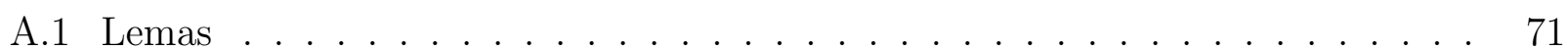

\begin{tabular}{lr}
\hline B Resultados & 85
\end{tabular}

B.1 Cálculo de $\bar{\beta}^{n}$. . . . . . . . . . . . . . . . . . . . . . . . . 85

B.2 Cálculo da primeira parte do Teorema 1 . . . . . . . . . . . . . . . . . . . . . . . 88

B.3 Resultados úteis . . . . . . . . . . . . . . . . . . . . . . . . . 90 


\section{Capítulo 1}

\section{Introdução}

Modelos multi-estados são mais comumente usados para descrever dados longitudinais que envolvem observações em série de uma mesma variável em um mesmo indivíduo e, geralmente, objetivam caracterizar mudanças da resposta de interesse ao longo do tempo. Os dados consistem de informações como tempo de ocorrência de eventos e tipos de eventos entre outras. Um evento pode ser representado por uma transição de um estado a outro e, quando vários eventos são observados, modelos multi-estados produzem uma modelagem adequada, pois sua estrutura é capaz de englobar dados coletados ao longo do tempo.

Os modelos multi-estados são utilizados em populações heterogêneas a partir da incorporação de covariáveis nas intensidades de transição, cujos efeitos podem, eventualmente, variar ao longo do tempo. Considere, por exemplo, que um tratamento invasivo (cirurgia e medicamento) seja comparado a outro não invasivo (somente medicamento). Pacientes que se submetem ao procedimento cirúrgico apresentam, em geral, comportamento distinto daqueles sob tratamento medicamentoso; o período pós-cirúrgico é considerado crítico, pois podem ocorrer complicações como infecções, arritmias, etc, aumentando consideravelmente o risco de óbito. Entretanto, passado esse período, em geral, ocorre a melhora em seu quadro clínico de forma que ambos os grupos tenderão a apresentar a mesma evolução após um certo período de tempo. Este cenário sugere a necessidade de se considerar modelagens que possibilitem a inclusão de efeitos dinânicos para covariáveis de interesse, representados por coeficientes 
dependentes do tempo.

Para motivar este trabalho consideramos um estudo conduzido na Faculdade de Medicina da USP, cujo objetivo foi comparar o desenvolvimento neuropsicomotor de recém-nascidos pré-termq ${ }^{1}$ (RNPT), com baixo risco de seqüelas neurológicas, com o desenvolvimento de recém-nascidos a termo (RNT). Detalhes podem ser obtidos em Restiffe (2007). O instrumento de avaliação utilizado é a escala AIMS (Alberta Infant Motor Scale), cujo escore varia de 0 a 58 e é adequada para avaliar bebês até 18 meses de idade. Os recém-nascidos são avaliados mensalmente desde o nascimento e, em cada caso, um escore lhes são atribuídos de acordo com os movimentos adquiridos. Quanto maior o número e a complexidade dos movimentos executados, maior é o escore. Os bebês são acompanhados até atingir o escore máximo (atribuído àqueles com capacidade de desenvolver todos os movimentos previstos na escala de avaliação) ou até 18 meses de idade cronológica (RNT) ou 18 meses de idade corrigidd $2^{2}$ (RNPT). Alguns dos bebês abandonam o estudo antes de seu término por perda de acompanhamento, causada por faltas consecutivas às avaliações ou mudança de endereço.

O desenvolvimento de RNPT é subestimado quando se usa a idade cronológica para comparar os escores observados com os escores da escala AIMS, causando preocupações desnecessárias a pais e médicos. Por outro lado, quando a idade corrigida é usada, o seu desenvolvimento pode ser superestimado. Dessa forma, existe a necessidade de conhecer até quando a idade do RNPT deve ser corrigida, de modo que o seu desenvolvimento seja adequadamente avaliado. Para responder a essa pergunta, é preciso conhecer quando ocorre o catch-up ${ }^{3}$ ou seja, o período de equiparação entre RNPT e RNT.

Uma possível abordagem para o problema descrito anteriormente é usar os modelos multiestados considerando cada etapa do desenvolvimento como um estado. Os bebês partem de um estado inicial, em que são considerados os movimentos mais simples que um recém-nascido pode executar até chegar ao último estado de desenvolvimento indicado na escala AIMS, que

\footnotetext{
${ }^{1}$ Nascidos antes da 37a. semana de idade gestacional.

${ }^{2}$ Obtida subtraindo-se o índice de prematuridade (IP-tempo faltante à idade gestacional para completar 40 semanas de gravidez) da idade cronológica (tempo de vida desde o nascimento).

${ }^{3}$ Quando o desenvolvimento do RNPT que se encontra em nível de desenvolvimento inferior equipara-se ao mesmo nível daquele usado como referência (RNT).
} 
corresponde a pegar o brinquedo no chão, de cócoras, e voltar a ficar em pé.

Espera-se que as intensidades de transição sejam distintas nas primeiras semanas de vida, mas devido ao catch-up, elas tendem a se igualar conforme os bebês avançam na idade. Assim, com a finalidade de descrever as mudanças que ocorrem na diferença de desenvolvimento neuropsicomotor entre os dois grupos de bebês, propomos um modelo multi-estados com coeficiente dependente do tempo.

Este trabalho está organizado da seguinte forma: no Capítulo 2, apresentamos a idéia geral de modelos multi-estados e introduzimos um modelo que engloba o coeficiente dependente do tempo. No Capítulo 3, apresentamos as propriedades do estimador proposto em grandes amostras. No Capítulo 4 aplicamos o modelo proposto a dados reais e geramos artificialmente uma amostra de tamanho maior considerando situações em que censuras são observadas e, finalmente, algumas conclusões são apresentadas no Capítulo 5. 


\section{Capítulo 2}

\section{Modelos multi-estados}

\subsection{Introdução}

Modelos multi-estados são utilizados para descrever sucessivas mudanças ou transições de estados experimentados por unidades amostrais. Modelos multi-estados são definidos a partir de um processo estocástico em que, a qualquer instante de tempo, um indivíduo ocupa um estado definido em um espaço de estados convenientemente construído. Os modelos multiestados podem ser vistos como uma generalização dos modelos de análise de sobrevivência clássica.

A progressão de doenças crônicas freqüentemente é descrita por níveis de severidade (exemplo: saúde, doença, doença com complicações e morte) conhecidos através de avaliações médicas. Esses níveis definem os estados e podem ser transitórios ou absorventes (Chiang, 1980). Denominam-se absorventes os estados em que não ocorrem transições partindo deles; uma vez que o indivíduo entra em um estado absorvente permanece para sempre nele. A morte é o exemplo mais comum de um estado absorvente.

Várias situações podem ser representadas por meio de modelos multi-estados. Kay (1986) usa essa abordagem em um estudo de câncer de mama, considerando vários estágios de desenvolvimento da doença, enquanto Marshall \& Jones (1995) os aplicam a dados de retinopatia 
diabética.

A situação mais simples em modelos multi-estados ocorre quando temos um estado absorvente que é representado por algum evento de interesse, geralmente morte, e um estado transiente. Ela é uma representação de análise de sobrevivência clássica e um esquema está dado na Figura 2.1.

\section{Vivo $\rightarrow$ Morto}

Figura 2.1: Esquema de análise de sobrevivência.

Um modelo que é muito estudado na literatura é conhecido como modelo doença-morte (Andersen, Borgan, Gill \& Keiding, 1993; Keiding, 1991) e sua representação é dada na Figura 2.2. Neste caso, um indivíduo pode transitar entre os estados de saúde e doença até entrar no

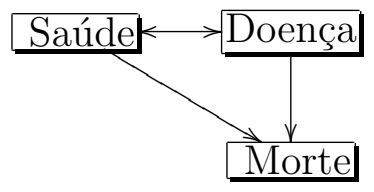

Figura 2.2: Esquema de doença-morte.

estado absorvente de morte.

O modelo multi-estados permite múltiplos estados transientes com um único estado absorvente, como mostrado na Figura 2.2, ou ainda um estado transiente e vários estados absorventes (Andersen \& Gill, 1982), como nos problemas de riscos competitivos representado na Figura 2.3. Esse modelo foi usado por Andersen, Abildstrom \& Rosthøj (2002) em um estudo

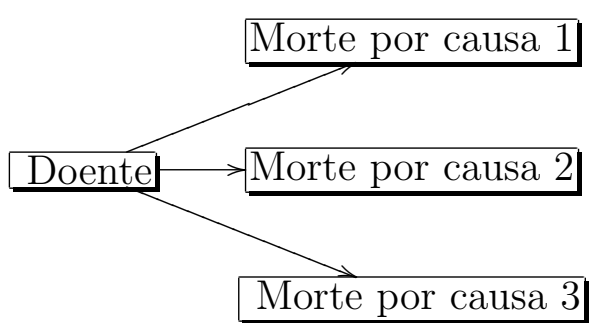

Figura 2.3: Esquema de riscos competitivos. 
de carcinogênese em ratos que podem morrer por diversas causas após receberem doses de radiação.

Frydman (1992) considera um modelo multi-estados para descrever a evolução de portadores de HIV até o desenvolvimento da AIDS, conforme mostra o esquema da Figura 2.4 . Usualmente, nesse caso, os pacientes são avaliados em intervalos de tempo pré-especificados, gerando observações periódicas. O modelo empregado nesse caso é denominado progressivo, pois uma vez que o paciente se encontra em um estado, ele poderá transitar somente para o estado mais avançado da doença.

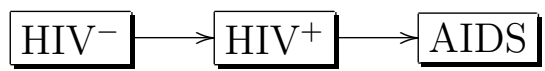

Figura 2.4: Processo de desenvolvimento da AIDS.

O modelo progressivo também pode ser usado para comparar o desenvolvimento de RNPT e RNT, descrito no Capítulo 1. Para isso, consideram-se os escores (ou conjunto de escores) como estados transitórios sendo o maior escore (evento de interesse) um estado absorvente. O processo é representado esquematicamente na Figura 2.5.

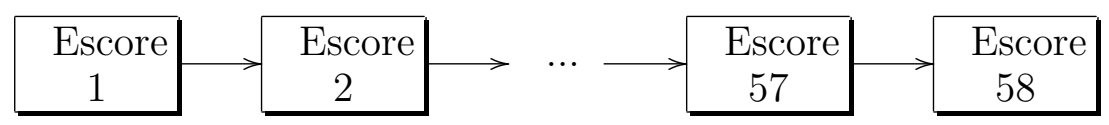

Figura 2.5: Desenvolvimento de recém-nascidos segundo a escala AIMS.

Um desenvolvimento teórico, baseado na teoria de processos de contagem, pode ser encontrado em Andersen et al. (1993). Em Hougaard (2000) também encontramos uma ampla revisão dos modelos multi-estados.

\subsection{Formulação do modelo}

Um processo multi-estados é um processo estocástico $\{S(t), t \in[0, \infty)\}$, em que $S(t)$ é uma variável aleatória que assume valores no conjunto de estados $\Gamma=\{1, \ldots, H\}$. O processo 
é caracterizado pela matriz de probabilidades de transição dada por

$$
\mathbf{P}(s, t)=\left(\begin{array}{ccccc}
P_{11}(s, t) & P_{12}(s, t) & \ldots & P_{1(H-1)}(s, t) & P_{1 H}(s, t) \\
P_{21}(s, t) & P_{22}(s, t) & \ldots & P_{2(H-1)}(s, t) & P_{2 H}(s, t) \\
\vdots & \vdots & \ddots & \vdots & \vdots \\
P_{(H-1) 1}(s, t) & P_{(H-1) 2}(s, t) & \ldots & P_{(H-1)(H-1)}(s, t) & P_{(H-1) H}(s, t) \\
P_{H 1}(s, t) & P_{H 2}(s, t) & \ldots & P_{H(H-1)}(s, t) & P_{H H}(s, t)
\end{array}\right)_{H \times H}
$$

em que

$$
P_{h j}(s, t)=P\left[S(t)=j \mid S(s)=h ; \mathcal{F}\left(s^{-}\right)\right]
$$

representa a probabilidade de um indivíduo se encontrar no estado $j$ no tempo $t$, dado que ele estava no estado $h$ no instante $s, \operatorname{com} s<t$ e $h, j=1, \ldots, H$, e dada a história do processo multi-estados $\mathcal{F}\left(s^{-}\right)$no instante imediatamente anterior a $s$, representada por $s^{-}$. Formalmente, $\mathcal{F}\left(s^{-}\right)$é uma $\sigma$-álgebra indexada por $t$ que cobre informações no intervalo $[0, t]$, e quando o tempo cresce, gera uma seqüência crescente de $\sigma$-álgebras conhecida como filtragem. Uma restrição que devemos considerar na matriz de probabilidade de transição é que cada linha da matriz $\mathbf{P}(s, t)$ tem soma igual a um, isto é, $\sum_{j} P_{h j}(s, t)=1$.

O processo multi-estados também pode ser caracterizado pela matriz de intensidades de transição

$$
\boldsymbol{\Lambda}(t)=\left(\begin{array}{ccccc}
\lambda_{11}(t) & \lambda_{12}(t) & \ldots & \lambda_{1(H-1)}(t) & \lambda_{1 H}(t) \\
\lambda_{21}(t) & \lambda_{22}(t) & \ldots & \lambda_{2(H-1)}(t) & \lambda_{2 H}(t) \\
\vdots & \vdots & \ddots & \vdots & \vdots \\
\lambda_{(H-1) 1}(t) & \lambda_{(H-1) 2}(t) & \ldots & \lambda_{(H-1)(H-1)}(t) & \lambda_{(H-1) H}(t) \\
\lambda_{H 1}(t) & \lambda_{H 2}(t) & \ldots & \lambda_{H(H-1)}(t) & \lambda_{H H}(t)
\end{array}\right)_{H \times H}
$$

em que $\lambda_{h j}(t)$ representa a intensidade de transição, isto é, a taxa instantânea de mudança de 
um estado $h$ para o estado $j$ no instante $t, t \geq 0$. Formalmente,

$$
\lambda_{h j}\left(t, \mathcal{F}\left(t^{-}\right)\right)=\lim _{\Delta t \rightarrow 0} \frac{P_{h j}(t, t+\Delta t)}{\Delta t} .
$$

Usando a restrição $\sum_{j} P_{h j}(s, t)=1$ e a definição de intensidade de transição em 2.2 , temos que $\sum_{j} \lambda_{h j}(s, t)=0$ e a diagonal da matriz 2.1 é,

$$
\lambda_{h h}(t)=-\sum_{j \neq h} \lambda_{h j}(t),
$$

em que $-\sum_{j \neq h} \lambda_{h j}(t)$ é a função de risco associada à distribuição do tempo de permanência no estado $h$, isto é,

$$
P_{h h}(t)=\exp \left\{-\int_{0}^{t} \sum_{j \neq h} \lambda_{h j}(u) d u\right\}
$$

A história $\mathcal{F}(t)$ do processo é gerada por $\{S(u), u \leq t\}$ e depende de

$$
\left\{N(t), T_{1}, \ldots, T_{N(t)}, S(0), S\left(T_{1}\right), \ldots, S\left(T_{N(t)}\right)\right\}
$$

em que $N(t)$ é o número de transições $h \rightarrow j$ em $[0, t]$ e $T_{m}$ é o $m$-ésimo tempo de transição $[m=1, \ldots, N(t)] . T_{N(t)}$ corresponde ao tempo da última transição observada no intervalo $[0, t]$.

Modelos multi-estados são, geralmente, baseados em processos de contagem multivariados, e se adaptam a modelagens mais complexas de eventos históricos (Andersen et al., 1993; Hougaard, 2000). Suposições são feitas em torno das leis de processo envolvidas. A suposição de Markov é a mais comum na maioria das aplicações (Aalen \& Johansen, 1978; Joly, Commenges, Helmer \& Letenneur, 2002). Até 1970, contudo, a atenção foi focada em modelos markovianos homogêneos. Modelos markovianos não homogêneos foram estudados por Fleming (1978), Aalen \& Johansen (1978), enquanto modelos semi-markovianos foram considerados por Lagakos, Sommer \& Zelen (1978), Joly \& Commenges (1999) e, mais recentemente, Tunes da Silva (2005). 
Um processo estocástico $\{S(t): t \in[0, \infty)\}$ é um processo markoviano se, para quaisquer $T_{0}<T_{1}<\ldots<T_{i}<\ldots<T_{j}$ e quaisquer inteiros $k_{0}, k_{1}, \ldots, k_{j}$,

$$
\begin{aligned}
& P\left\{S\left(T_{j}\right)=k_{j} \mid S\left(T_{0}\right)=k_{0}, S\left(T_{1}\right)=k_{1}, \ldots, S\left(T_{j-1}\right)=k_{j-1}\right\}= \\
& P\left\{S\left(T_{j}\right)=k_{j} \mid S\left(T_{j-1}\right)=k_{j-1}\right\} .
\end{aligned}
$$

Diz-se que o processo multi-estados obedece a um modelo markoviano quando a história $\mathcal{F}\left(t^{-}\right)$pode ser esquecida, isto é, quando

$$
\lambda_{h j}\left(t, \mathcal{F}\left(t^{-}\right)\right)=\lambda_{h j}(t), \quad h, j=0, \ldots, H ; h \neq j .
$$

Define-se a intensidade acumulada de transição do estado $h$ para o estado $j$ como $\Lambda_{h}((0, t])=$ $\int_{0}^{t} \lambda_{h j}(u) \mathrm{d} u, t \geq 0$. Sob o modelo markoviano, dado $S\left(T_{i}\right)$ (presente), a distribuição de probabilidade condicional de $S\left(T_{j}\right)$ (futuro) é independente de $\left\{S\left(T_{0}\right), \ldots, S\left(T_{i-1}\right)\right\}$ (passado).

O modelo markoviano é dito não homogêneo quando as taxas instantâneas de transição dependem do tempo $t$ desde a origem do processo. Com a suposição mais restritiva de que a intensidade de transição não depende de $t$, isto é, $\lambda_{h j}(t)=\lambda_{h j}$, o modelo markoviano é dito homogêneo. Nesse caso, a probabilidade de transição depende somente da diferença $(t-s) \mathrm{e}$ não de $t$ e $s$ separadamente. Nesse contexto, as intensidades de transição não dependem do tempo gasto no estado atual.

Quando assumimos que a intensidade de transição depende do tempo gasto no estado atual, o modelo é chamado semi-markoviano e $\lambda_{h j}\left(t, \mathcal{F}\left(t^{-}\right)\right)=\lambda_{h j}\left(t-T_{N\left(t^{-}\right)}\right)$. Usualmente, modelos semi-markovianos são utilizados para modelar o tempo de permanência em um determinado estado.

Segundo Commenges (1999), os modelos não homogêneos e semi-markovianos são comparáveis em termos de flexibilidade. A escolha entre os dois depende do que se pretende modelar: a intensidade de transição desde o início do processo ou o tempo de permanência em um determinado estado. Por exemplo, o risco de AIDS essencialmente depende do tempo desde a infecção; por isso Joly \& Commenges (1999) escolheram um modelo semi-markoviano; contudo, nem sempre a escolha é óbvia. 
A propriedade markoviana (2.4) implica importantes relações entre as probabilidades de transição $P_{h j}(s, t)$. Se fixamos um ponto $\xi$ no intervalo $[s, t]$, tal que $s<\xi<t$, e consideramos as respectivas variáveis aleatórias $S(s), S(\xi), S(t)$, então

$$
\begin{array}{r}
P_{h j}(s, t)=\sum_{i} P_{h i}(s, \xi) P_{i j}(\xi, t), \\
\text { com } \quad h, j=1,2, \ldots \quad \text { e } \quad s<\xi<t .
\end{array}
$$

A relação entre a matriz de probabilidades de transição $\mathbf{P}(s, t)$ e a matriz de intensidade de transição $\Lambda(t)$ é dada pelas equações diferenciais de Kolmogorov (Chiang, 1980),

$$
\frac{\partial}{\partial t} \mathbf{P}(s, t)=\mathbf{P}(s, t) \boldsymbol{\Lambda}(t), \quad \text { com } P_{h j}(s, t)=P[S(t)=j \mid S(s)=h] .
$$

As probabilidades de transição são as soluções para as equações diferenciais em (2.6). Considerando $\boldsymbol{\Lambda}(t)=\boldsymbol{\Lambda}$ constante em relação ao tempo (modelo homogêneo), a equação (2.6) pode ser substituída por

$$
\frac{\partial}{\partial t} \mathbf{P}(0, t)=\mathbf{P}(0, t) \boldsymbol{\Lambda}, \quad \text { com } P_{h j}(0, t)=P[S(t)=j \mid S(0)=h],
$$

que admite uma única solução:

$$
\mathbf{P}(0, t)=\exp \{\boldsymbol{\Lambda} t\}=\sum_{r=0}^{\infty} \frac{\boldsymbol{\Lambda}^{r} t^{r}}{r !}, \quad \operatorname{com} \boldsymbol{\Lambda}^{0}=\mathbf{I}
$$

Pode-se mostrar que a série de matrizes em (2.8) converge unifomemente em $t$ (Chiang, 1980).

Se B é uma matriz quadrada cuja $j$-ésima coluna contém autovetores associados ao autovalor $\rho_{j}$ da matriz de intensidade $\boldsymbol{\Lambda}$, então

$$
\mathbf{P}(0, t)=\mathbf{B} \operatorname{diag}\left\{e^{\rho_{1} t, \ldots, \rho_{H} t}\right\} \mathbf{B}^{-1}
$$

é a solução explícita de (2.6) para os modelos de Markov homogêneos [ver demonstrações em Chiang (1980)]. Entretanto, a mesma solução não pode ser aplicada diretamente para o 
modelo não homogêneo, pois a matriz $\Lambda(t)$ não é constante nesse caso.

Quando todos indivíduos da população alvo têm a mesma intensidade de transição, diz-se que a população é homogênea. Entretanto, em algumas situações a população é heterogênea e nesse caso é razoável dividí-la em subgrupos de forma que a homogeneidade seja obtida. O modelo homogêneo pode ser estendido para essas populações heterogêneas através da inclusão de covariáveis. Por exemplo, o elemento $(h, j)$ da matriz de intensidade de transição $\Lambda=\Lambda(X)$ pode ser escrito como

$$
\lambda_{h j}(\mathbf{X})=\alpha_{0 h j} e^{\boldsymbol{\beta}_{h j}^{\prime} \mathbf{X}}, \quad h, j=1,2, \ldots, H
$$

em que $\boldsymbol{\beta}_{h j}$ é o vetor de coeficientes de regressão associado ao vetor de covariáveis $\mathbf{X}$, para a transição $h \rightarrow j$, e $\alpha_{0 h j}$ é a função de risco base. O modelo 2.10 se assemelha ao modelo de riscos proporcionais de Cox, em que $\alpha_{0 h j}$ não é especificado.

Os modelos homogêneos são simples e fáceis de aplicar, contudo não se ajustam a todas as situações práticas, pois nem sempre podemos considerar a intensidade de transição constante ao longo de um processo. Assim, vários autores têm empregado outros modelos, como os semi-markovianos e os não homogêneos.

Tunes da Silva (2005), por exemplo, usa o modelo semi-markoviano para estimar a esperança do tempo de sobrevivência ajustado pela qualidade de vida por meio da modelagem de tempos de permanência.

Sob um modelo markoviano não homogêneo, Frydman (1992) usa um processo de estimação não paramétrico através do estimador autoconsistente (Cox \& Oakes, 1984) para um estudo de AIDS em que são considerados três estados (1: HIV negativo, 2: HIV positivo e 3: AIDS). As avaliações ocorrem periodicamente, e o tempo de entrada no segundo estado é desconhecido, ou seja, corresponde a censura intervalar. Nesse caso, sabe-se apenas que a transição $1 \rightarrow 2$ ocorreu em um dado intervalo de tempo. Os tempos de transição $2 \rightarrow 3$ ou são conhecidos ou são censurados à direita. Ela assume que o esquema de amostragem é não informativo, isto é, os tempos amostrados para a realização das avaliações são considerados aleatórios, como discutido em Grüger, Kay \& Schumacher (1991), e que a intensidade de transição do estado 2 
para o 3 só depende do tempo cronológico $t$.

Frydman (1992) propõe uma estimação conjunta da função de distribuição do tempo no estado $1(F)$ e da intensidade acumulada de transição do estado 2 para $3\left(\Lambda_{2}((0, t])\right)$. Um estimador autoconsistente de $\left(F, \Lambda_{2}((0, t])\right)$ é definido, e ela mostra que o estimador de máxima verossimilhança de $\left(F, \Lambda_{2}((0, t])\right)$ é autoconsistente (Cox \& Oakes, 1984), porém as propriedades desse estimador não são apresentadas.

Frydman (1995) parte de um modelo semi-markoviano supondo que a intensidade de transição $1 \rightarrow 2$ depende somente do tempo $t$ desde de o início do processo, enquanto a intensidade de transição de $2 \rightarrow 3$ depende do tempo $T_{N(t)}$ de entrada no estado 2 , além do tempo $t$, e usa o seguinte modelo semi-paramétrico para abordar o problema:

$$
\lambda_{23}\left(t-T_{N(t)}\right)=\frac{\lambda(t) e^{\beta\left(t-T_{N(t)}\right)}}{1+\lambda(t) e^{\beta\left(t-T_{N(t)}\right)}},
$$

em que $\lambda_{23}\left(t-T_{N(t)}\right)$ é a intensidade de transição do estado 2 para o estado 3 , $\beta$ é o coeficiente da covariável $\nu=t-T_{N(t)}$ e $\lambda(t)$ é o fator que leva em conta o tempo desde o início do processo.

Hsieh, Chen \& Chang (2002) e Pérez-Ocón, Ruiz-Castro \& Gámiz-Pérez (2001) consideram modelos não homogêneos usando o modelo exponencial segmentado (piecewise exponential) e o modelo Weibull segmentado (piecewise Weibull) em estudos de câncer de mama com três estados (1: normal, 2: fase de detecção e 3: fase clínica). Eles consideram que as intensidades de transição se diferenciam não só entre as transições $h \rightarrow j$, mas também de acordo com a idade $t^{*}$ do processo. Por exemplo, usando o modelo exponencial segmentado, Hsieh et al. (2002) consideram as intensidades de transição modeladas como

$$
\begin{gathered}
\begin{cases}\lambda_{12}=\lambda_{1} e^{\boldsymbol{\beta}_{1} \mathbf{X}}, & t \leq t^{*} \\
\lambda_{12}^{*}=\lambda_{1}^{*} e^{\boldsymbol{\beta}_{1}^{*} \mathbf{X}}, & t>t^{*}\end{cases} \\
\begin{cases}\lambda_{23}=\lambda_{2} e^{\boldsymbol{\beta}_{2} \mathbf{X}}, & t \leq t^{*} \\
\lambda_{23}^{*}=\lambda_{2}^{*} e^{\boldsymbol{\beta}_{2}^{*} \mathbf{X}}, & t>t^{*} .\end{cases}
\end{gathered}
$$


Nesse exemplo, $t^{*}$ corresponde à idade aproximada da menopausa. Para mulheres com idade inferior a 50 anos, a intensidade de transição do estado 1 para o estado 2 é representada por $\lambda_{12}$ e, para aquelas com idade superior a 50 , a transição é representada por $\lambda_{12}^{*}$.

Outro estimador, conhecido como estimador Nelson-Aalen (Andersen et al., 1993), é muito usado para os modelos não homogêneos. Seja a intensidade acumulada de transição denotada por $\Lambda_{h j}(t)=\int_{0}^{t} \lambda_{h j}(u) \mathrm{d} u$ e o seu estimador por $\widehat{\Lambda}_{h j}(t)$. A probabilidade de transição ocorrida do tempo $s$ para $t$ pode ser escrita com uma integral-produto (Gill \& Johansen, 1990),

$$
\mathbf{P}(s, t)=\underset{u \in(s, t]}{\boldsymbol{\pi}}(\mathbf{I}+\mathrm{d} \mathbf{A}(u)),
$$

em que I é a matriz identidade de dimensão $H$ e $\mathbf{A}(u)$ denota a matriz de intensidades acumuladas de transição. $\boldsymbol{\pi}$ é a versão para a variável contínua do produtório $\prod$, assim como a integral $\int$ generaliza o somatório $\sum$ [ver Gill \& Johansen (1990)]. A probabilidade de transição (2.11) é estimada por

$$
\widehat{\mathbf{P}}(s, t)=\underset{u \in(s, t]}{\boldsymbol{\pi}}(\mathbf{I}+\mathrm{d} \widehat{\mathbf{A}}(u)),
$$

em que $\widehat{\mathbf{A}}(u)$ é o estimador de $\mathbf{A}(u)$ baseado no estimador Nelson-Aalen, no caso sem covariáveis. Sob o modelo mais simples de dois estados para dados de sobrevivência, o estimador $\widehat{\mathbf{P}}(0, t)$ fica reduzido ao clássico estimador de Kaplan-Meier (Kaplan \& Meier, 1958).

Quando queremos avaliar o efeito das covariáveis, podemos estender as probabilidades de transição para o cenário de regressão, fazendo

$$
\mathbf{P}(s, t)=\underset{u \in(s, t]}{\boldsymbol{\pi}}(\mathbf{I}+\mathrm{d} \mathbf{A}(u ; \mathbf{X})),
$$

em que $\Lambda(u ; \mathbf{X})$ denota a matriz de intensidade acumulada, dado o vetor de covariáveis $\mathbf{X}$. Um exemplo é o modelo aditivo não paramétrico de Aalen (1989),

$$
\lambda_{h j i}(t)=\lambda_{h j 0}(t)+\beta_{h j}(t) X_{i},
$$


em que $\lambda_{h j 0}(t)$ é a função de risco base e $\beta_{h j}(t)$ é o coeficiente de regressão para covariável $X$.

Nesse modelo, tanto a intensidade de transição base como a função de regressão $\beta_{h j}(t)$ não são especificadas e estimativas não paramétricas podem ser obtidas usando o procedimento de mínimos quadrados generalizados. Aalen, Borgan \& Fekjaer (2001) estudam o estimador produto limite baseado no modelo de regressão aditivo (2.14).

No exemplo de bebês RNPT e RNT, que motivou nosso estudo, espera-se que a diferença do desenvolvimento neuropsicomotor dos dois grupos diminua com o avanço da idade. Assim, de modo semelhante ao modelo 2.14, vamos considerar um modelo não homogêneo, que permita a variação da intensidade de transição no tempo. Porém, em vez da forma aditiva utilizada no modelo (2.14), adotaremos a forma multiplicativa, estendendo o usual modelo de Cox para modelos multi-estados.

\subsection{Coeficientes dependentes do tempo}

Zucker \& Karr (1990), Murphy \& Sen (1991), Hastie \& Tibshirani (1993), Murphy (1993), Marzec \& Marzec (1997), Pedroso de Lima \& Sen (1999) e Martinussen, Scheike \& Skovgaard (2002) abordam generalizações do modelo de Cox, $\lambda(t \mid \mathbf{X})=\alpha_{0}(t) e^{\boldsymbol{\beta}^{\prime} \mathbf{X}}$, em que os coeficientes são dependentes do tempo. Mais especificamente, consideram modelos do tipo,

$$
\lambda(t, \mathbf{X})=\alpha_{0}(t) e^{\boldsymbol{\beta}^{\prime}(t) \mathbf{X}},
$$

em que $\alpha_{0}(t)$ é uma função não negativa, desconhecida e que atua multiplicativamente na intensidade de transição e $\boldsymbol{\beta}(t)$ é o vetor de coeficientes dependentes do tempo para o vetor de covariáveis $\mathbf{X}$. Como $\boldsymbol{\beta}^{\prime}(t)$ não é constante, esse modelo representa riscos não proporcionais.

O modelo (2.15) é mais flexível e descreve informações mais detalhada do efeito da covariável que o usual modelo de Cox. Para estimar o coeficiente no modelo (2.15), Murphy \& Sen (1991) propõem um processo de estimação de crivo (sieve). Um crivo é uma seqüência $\left\{\Theta_{K_{n}}, n \geq 1\right\}$ de subconjuntos de dimensão finita de um espaço paramétrico de dimensão infinita $\Theta$, tal que $\bigcup_{n \geq 1} \Theta_{K_{n}}$ seja denso em $\Theta$. A idéia consiste em aproximar a função $\beta(t)$ 
por $\sum_{k=1}^{K} \beta_{k} \mathbb{I}_{k}(u)$, em que $\mathbb{I}_{k}(u)$ é a função indicadora do $k$-ésimo intervalo de tempo e mostrar que este converge uniformemente para $\beta(t)$ quando o número de intervalos $K$ vai para $+\infty$.

A Figura 2.6 é um exemplo de estimador de crivo com cinco intervalos para a função $\beta(t)$.

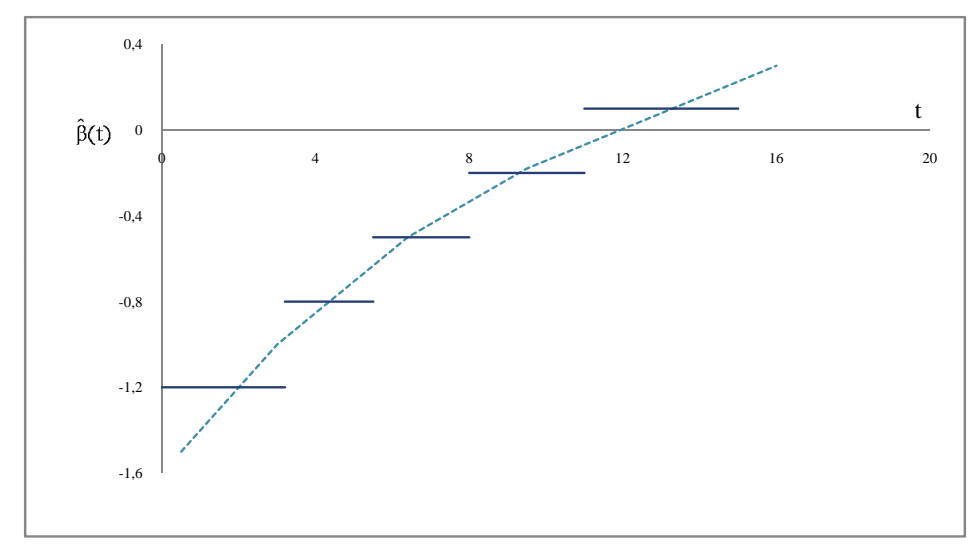

Figura 2.6: Exemplo do estimador histograma crivo.

Murphy \& Sen (1991) discutem a convergência do estimador de crivo e provam um teorema limite central funcional para o estimador de crivo integrado. Ainda baseados nesse estimador, Murphy (1993) e Marzec \& Marzec (1997) discutem a qualidade do ajuste do modelo 2.15), enquanto Pedroso de Lima \& Sen (1999) estendem o modelo para o caso de análise de sobrevivência bivariado.

\subsection{Especificação do modelo}

O modelo 2.15 de Murphy \& Sen (1991) não engloba o caso multi-estados. Seguindo a mesma linha de seus estudos, vamos estender o modelo de Cox com coeficiente dependente do tempo e usar o estimador de crivo (Grenander, 1981) para os modelos multi-estados. 
Considere que $n$ indivíduos são acompanhados e que a cada um deles seja associado um vetor de processo de contagem $N_{h}(t)=\left(N_{h 1}(t), \ldots, N_{h n}(t)\right)^{\prime}, t \in[0, \tau], \tau<\infty, N_{h i}(t)$ nada mais é do que uma função indicadora, assumindo o valor 0 a partir de $t=0$ e assumindo o valor 1 quando ocorre a transição do tipo $h$. Transição do tipo $h$ referem-se a todas as possíveis transições, no caso particular do modelo progressivo, refere-se a transições $h \rightarrow h+1$, com $h=1, \ldots, H-1$, em que $H$ representa o número de estados. Desta forma, este processo vale 1 se uma transição $h \rightarrow h+1$ tiver ocorrido antes de ou em $t$. A especificação probabilística para o processo é dado por

$$
\lambda_{h}(t, \mathbf{X})=\alpha_{0 h}(t) e^{\boldsymbol{\beta}^{\prime}(t) \mathbf{X}(t)} Y_{h}(t), \quad h=1, \ldots, H-1,
$$

em que $\alpha_{0 h}(t)$ denota a intensidade de transição basal para transição do tipo $h$ e $\boldsymbol{\beta}(t)$ é o coeficiente dependente do tempo para o vetor de covariáveis $\mathbf{X}(t)$. $\boldsymbol{\beta}(t)$ e $\alpha_{0 h}(t)$ são funções determinísticas em $[0, \tau], X(t)=\left(X_{1}(t), \ldots, X_{n}(t)\right)$ é o vetor de covariáveis dependentes do tempo e $Y_{h}(t)=\left(Y_{h 1}(t), \ldots, Y_{h n}(t)\right)$ é um vetor observado de processo estocástico previsível, ou seja, $Y_{h i}(t)$ é o indicador de que o indivíduo $i$ está em risco no tempo $t^{-}$para transição do tipo $h$. Note que o coeficiente $\boldsymbol{\beta}(t)$ é comum a todas as transições.

No exemplo de desenvolvimento de bebês, $\beta(t)$ descreve as mudanças do efeito de grupo ocorridas ao longo do estudo. Se $\beta(t)=0$ temos ausência de efeito de grupo, no instante $t$, isto é, o desenvolvimento neuropsicomotor de RNPT e RNT é semelhante. $\beta(t)>0$ indica que o desenvolvimento dos RNPT é mais rápido que os RNT, enquanto $\beta(t)<0$ indica que os RNPT se desenvolvem mais lentamente que os RNT. Além disso, $\beta(t)$ pode ser interpretado como logaritmo da razão das intensidades de transição entre os grupos de bebês RNPT e RNT para transitar de um estado a outro para um tempo $t$ fixo.

$N_{h i}(t)$ são funções tipo escada, contínuas à direita, com valor zero no tempo zero, com saltos de tamanho 1 , tais que dois componentes nunca saltam simultaneamente. $\Lambda_{h}(t)$ é chamado compensador de $N_{h}(t)$, de forma que $N_{h}(t)=\Lambda_{h}(t)+M_{h}(t)$, em que $M_{h}(t)=$ $\left(M_{h 1}(t), \ldots, M_{h n}(t)\right)$ são martingais de média zero. Supondo que $\Lambda_{h}(t)$ seja absolutamente 
contínua, temos

$$
\Lambda_{h}(t)=\int_{0}^{t} \lambda_{h}(s) \mathrm{d} s, \quad t \geq 0
$$

que é o processo de intensidade integrado.

Uma quantidade necessária nesta teoria é o processo de variação previsível de $M_{h}(t)$ dado por

$$
\left\langle M_{h i}^{n}(t), M_{h j}^{n}(t)\right\rangle=\left\{\begin{aligned}
\int_{0}^{t} \lambda_{h i}(u) \mathrm{d} u, & \text { para } i=j \\
0, & \text { para } i \neq j .
\end{aligned}\right.
$$

Esta quantidade é definida como compensador do processo $M_{h}^{2}(t)$.

Uma revisão mais detalhada sobre processos de contagem pode ser encontrada em Andersen et al. (1993).

No próximo capítulo são apresentadas propriedades assintóticas baseadas no modelo (2.16). 


\section{Capítulo 3}

\section{Teoria assintótica}

Neste capítulo são apresentadas propriedades assintóticas do estimador histograma crivo (histogram sieve) quando consideramos o modelo (2.16) com coeficiente dependente do tempo em um modelo multi-estados, estendendo os resultados obtidos em Murphy \& Sen (1991). A Subseção 3.2.1 contém as suposições necessárias para demonstrações dos teoremas referentes às propriedades assintóticas. A consistência fraca é provada na Subseção $3.2 .2 \mathrm{e}$, em seguida, nas Subseções 3.2.3 e 3.2.4 são apresentados o teorema central do limite funcional para o coeficiente de regressão integrado e a prova da consistência para o estimador do processo de variação, respectivamente.

\subsection{Verossimilhança}

Seja o modelo

$$
\lambda_{h i}(t, X)=\alpha_{0 h i}(t) e^{\beta^{\prime}(t) \mathbf{X}_{i}(t)} Y_{h i}(t), \quad h=1, \ldots, H-1, \quad i=1, \ldots, n,
$$

em que $\alpha_{0 h i}(t)$ é a função de risco basal do indivíduo $i$ para transição do tipo $h$ e $\boldsymbol{\beta}(t)$ é o vetor de coeficientes dependentes do tempo para o vetor de covariáveis $\mathbf{X}_{i}(t)$. A inferência 
para $\boldsymbol{\beta}(t)$ é baseada em um produto de verossimilhanças parciais de Cox,

$$
\begin{aligned}
\mathcal{L}(\beta) & =\underset{t \in[0, \tau]}{\boldsymbol{\pi}} \prod_{i=1}^{n} \prod_{h=1}^{H-1}\left(\frac{\lambda_{h i}(t)}{\sum_{j=1}^{n} \lambda_{h j}(t)}\right)^{\mathrm{d} N_{h i}(t)} \\
& =\underset{t \in[0, \tau]}{\prod_{i=1}} \prod_{h=1}^{n} \prod^{H-1}\left(\frac{\alpha_{0 h i}(t) e^{\beta(t) X_{i}(t)} Y_{h i}(t)}{\sum_{j=1}^{n} \alpha_{0 h j}(t) e^{\beta(t) X_{j}(t)} Y_{h j}(t)}\right)^{\mathrm{d} N_{h i}(t)} \\
& =\underset{t \in[0, \tau]}{\boldsymbol{\pi}} \prod_{i=1}^{n} \prod_{h=1}^{H-1}\left(\frac{e^{\beta(t) X_{i}(t)} Y_{h i}(t)}{\sum_{j=1}^{n} e^{\beta(t) X_{j}(t)} Y_{h j}(t)}\right)^{\mathrm{d}(t)}, \quad \tau \geq 0 .
\end{aligned}
$$

A log-verossimilhança é dada por

$$
\begin{aligned}
\log \mathcal{L}(\beta) & =\sum_{i=1}^{n} \sum_{h=1}^{H-1} \int_{0}^{\tau} \ln \left[\frac{e^{\beta(u) X_{i}^{n}(u)}}{\sum_{j=1}^{n} e^{\beta(u) X_{i}^{n}(u)} Y_{h j}^{n}(u)}\right] \mathrm{d} N_{h i}^{n}(u) \\
& =\sum_{i=1}^{n} \sum_{h=1}^{H-1} \int_{0}^{\tau}\left[\beta(u) X_{i}^{n}(u)+\ln \frac{1}{\sum_{j=1}^{n} e^{\beta(u) X_{j}^{n}(u)} Y_{h j}^{n}(u)}\right] \mathrm{d} N_{h i}^{n}(u), \quad \tau \geq 0,
\end{aligned}
$$

que corresponde à log-verossimilhança parcial para o modelo multi-estados apresentada em Andersen et al. (1993), exceto pelo coeficiente dependente do tempo. Se considerarmos apenas dois estados, que é o caso particular da análise de sobrevivência, a verossimilhança (3.1) corresponde àquela utilizada em Murphy \& Sen (1991).

Note que, sendo $\beta(t)$ um coeficiente dependente do tempo, seu espaço paramétrico torna-se de dimensão infinita, o que impossibilita a maximização direta de $\mathcal{L}_{n}(\beta)$ [ ver Zucker \& Karr (1990)]. Nessas situações, Murphy \& Sen (1991) sugerem o método de crivos (Grenander, 1981). A idéia é particionar o intervalo de tempo [0, $\tau]$ em $K_{n}$ subintervalos e maximizar a verossimilhança nesse conjunto. Esse método assegura a convergência fraca do estimador de máxima verossimilhança parcial (EMVP) $\widehat{\beta}_{K_{n}}$ para o verdadeiro valor $\beta_{0} \in \Theta$. A essência do método consiste em aumentar o espaço paramétrico $\Theta_{K_{n}}, n \geq 1$, aumentando o número de intervalos $K_{n}$ com uma taxa que permita que $\beta$ seja consistente.

O espaço paramétrico baseado em uma partição de tamanho $K_{n}$ associado ao histograma 
crivo é representado por

$$
\Theta_{K_{n}}=\left\{\beta: \beta(u)=\sum_{k=1}^{K_{n}} \beta_{k} \mathbb{I}\left\{u \in I_{k}^{n}\right\}, \operatorname{para}\left(\beta_{1}, \ldots, \beta_{K_{n}}\right) \in \mathbb{R}^{K_{n}}\right\},
$$

em que $\mathbb{I}_{k}^{n}(u)$ é a função indicadora do intervalo $I_{k}^{n}$; Os elementos $\left(I_{1}^{n}, \ldots, I_{K_{n}}^{n}\right)$ representam segmentos consecutivos do intervalo $[0, \tau]$ cujos comprimentos são denotados por $\mathbf{l}=\left(l_{1}^{n}, \ldots, l_{K_{n}}^{n}\right)$. $l_{(1)}^{n}$ e $l_{\left(K_{n}\right)}^{n}$ são os comprimentos mínimo e máximo, respectivamente, e $\|l\|=\sqrt{\sum_{k=1}^{K_{n}} l_{k}^{2}}$ representa a norma do vetor $\mathbf{l}$. Note que o número de intervalos $K_{n}$ depende do tamanho amostral $n$, isso significa que $K_{n}$ aumenta de acordo com aumento de $n$ de forma que assegure tanto a consistência como a eficiência dos estimadores de máxima verossimilhança parcial.

O verdadeiro valor do parâmetro, $\beta_{0}(t)$, e seu estimador de máxima verossimilhança $\widehat{\beta}^{n}$ não são diretamente comparáveis, pois pertencem a diferentes espaços paramétricos. Assim, existe a necessidade de um representante de $\beta_{0}$ no espaço do estimador crivo que denominamos $\bar{\beta}^{n}$ e o seu estimador de máxima verossimilhança é $\widehat{\beta}^{n}$. A Figura 3.1 ilustra uma situação bem simplificada, em que $\beta_{0}$ está representado em um espaço paramétrico de dimensão três e o estimador de crivo em um espaço de dimensão dois. A Figura 3.1 mostra que à medida

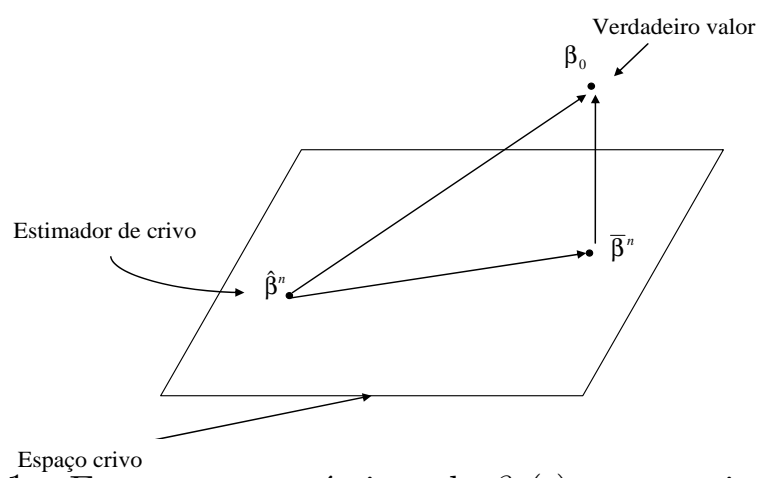

Figura 3.1: Espaçocrivo

que $n$ cresce, $\bar{\beta}^{n}$ se aproxima de $\beta_{0}(t)$ ao mesmo tempo em que $\widehat{\beta}^{n}$ se aproxima de $\bar{\beta}^{n}$ e consequentemente $\widehat{\beta}^{n}$ se aproxima de $\beta_{0}(t)$. Assim, vamos considerar a quantidade $\bar{\beta}^{n} \in \Theta_{K_{n}}$, tal que, quando $K_{n} \rightarrow \infty$, tem-se $\bar{\beta}^{n} \rightarrow \beta_{0}$, uniformemente em $t$. Sendo assim, $\bar{\beta}^{n}$ deve ser 
cuidadosamente selecionado, e uma escolha adequada é sugerida por Murphy \& Sen (1991) para o caso univariado, cuja idéia é expandir a função log-verossimilhança em torno de um ponto de $\Theta_{K_{n}}$, no lugar de expandir em torno de $\beta_{0}$. Usando os mesmos princípios, consideremos em cada intervalo um valor para $\bar{\beta}^{n}$ que é uma função $\beta_{0}(u)$, isto é,

$$
\bar{\beta}^{n}(u)=\sum_{k=1}^{K_{n}} \bar{\beta}_{k}^{n} \mathbb{I}_{k}(u)
$$

para $u \in[0, \tau]$, em que

$$
\begin{aligned}
\bar{\beta}_{k}^{n}= & \sum_{h=1}^{H-1} \frac{\int_{0}^{\tau} \mathbb{I}_{k}(u) \beta_{0}(u) v_{n h}\left(\beta_{0}, u\right) s_{n h}^{(0)}\left(\beta_{0}, u\right) \alpha_{0 n h}(u) \mathrm{d} u}{\sigma_{k}^{2}}, \\
\operatorname{com} \sigma_{k}^{2}= & \sum_{h=1}^{H-1} \sigma_{k h}^{2}=\sum_{h=1}^{H-1} \int_{0}^{\tau} \mathbb{I}_{k}(u) v_{n h}\left(\beta_{0}, u\right) s_{n h}^{(0)}\left(\beta_{0}, u\right) \alpha_{0 n h}(u) \mathrm{d} u, \\
& k=1, \ldots, K_{n} .
\end{aligned}
$$

A expressão para $\bar{\beta}_{k}^{n}$ é semelhante àquela de Murphy \& Sen (1991) [ver detalhes na Seção B.1, exceto pela inclusão de somatórios para adequar ao nosso contexto multi-estados, mas ainda assim, pode ser interpretado como uma média ponderada de $\beta_{0}(u)$, dentro de cada elemento da partição (as demais quantidades serão definidas na próxima seção). Além disso, é possível observar que $\alpha_{0 h}(u)$ e $\beta_{0}(u)$ não se alteram com o tamanho amostral $n$.

\subsection{Propriedades assintóticas do estimador de crivo}

Para demonstrar a consistência do estimador de máxima verossimilhança, é necessário definir as quantidades a seguir, tradicionalmente consideradas para o modelo de Cox e aqui generalizadas para o caso multi-estados. 
Para cada $u \in[0, \tau]$ e para cada tipo de transição $h(h=1, \ldots, H-1)$,

$$
\begin{aligned}
S_{n h}^{(i)}(\beta, u) & =\frac{1}{n} \sum_{j=1}^{n} e^{\beta(u) X_{j}^{n}(u)}\left(X_{j}^{n}(u)\right)^{i} Y_{j h}^{n}(u), \quad i=1,2,3,4, \\
\mathbb{E}_{n h}(\beta, u) & =\frac{S_{n h}^{(1)}(\beta, u)}{S_{n h}^{(0)}(\beta, u)} \\
\mathbb{V}_{n h}(\beta, u) & =\frac{S_{n h}^{(2)}(\beta, u)}{S_{n h}^{(0)}(\beta, u)}-\left(\mathbb{E}_{n h}(\beta, u)\right)^{2},
\end{aligned}
$$

e para $\beta \in \Theta_{K_{n}}$,

$$
\|\beta\|^{2}=\sum_{k=1}^{K_{n}} \beta_{k}^{2} .
$$

Note que $\alpha_{0 h}(t)$ e $\beta_{0}(t)$ são funções que não dependem do tamanho amostral $n$. Nas notações que se seguem, são omitidos os índices $n$. Além disso, necessitamos das condições de regularidade representadas pelas suposições a seguir, que nada mais são do que generalizações daqueles usualmente exigidos no modelo de Cox.

\subsubsection{Suposições}

Suposição A (Estabilidade Assintótica)

Existe $s_{h}^{(i)}\left(\beta_{0}, u\right), i=0,1,2$, tal que

1. $\sup _{u \in[0, \tau]}\left|S_{h}^{(i)}\left(\beta_{0}, u\right)-s_{h}^{(i)}\left(\beta_{0}, u\right)\right|=o_{p}(1)$,

2. $n \int_{0}^{\tau}\left|S_{h}^{(i)}\left(\beta_{0}, u\right)-s_{h}^{(i)}\left(\beta_{0}, u\right)\right|^{2} \mathrm{~d} u=O_{p}(1) \quad$ e

3. existe $\gamma>0$ tal que

$$
\sup _{u \in[0, \tau]} \sup _{\substack{b \in \mathbb{R} \\\left|b-\beta_{0}(u)\right|<\gamma}} \frac{\left|S_{h}^{(i)}(b, u)\right|}{S_{h}^{(0)}(b, u)}=O_{p}(1), \quad i=1,2,3,4 .
$$


Suposição B (Condição de Lindeberg)

Existe $\delta_{h}>0$ para os quais

$$
\max _{1 \leq j \leq n} \int_{0}^{\tau} \mathbb{I}\left\{u:\left|X_{j}(u) Y_{j h}(u)\right|>\frac{1}{2} \epsilon \sqrt{n}, \beta_{0}(u) X_{j}(u)>-\delta_{h}\left|X_{j}(u)\right|\right\} \mathrm{d} u=o_{p}(1),
$$

para cada $\epsilon>0$.

Suposição C (Regularidade Assintótica)

1. Existem constantes $U_{1}>0, U_{2}>0$ tal que

$\max \left\{\alpha_{0 h}(u), s_{h}^{(i)}\left(\beta_{0}, u\right), i=0,1,2\right\} \leq U_{1} \quad \mathrm{e}$

$s_{h}^{(0)}\left(\beta_{0}, u\right) \geq U_{2}$, uniformemente em $u \in[0, \tau]$.

2. Existe uma constante $L>0$, tais que para

$$
\begin{aligned}
& v_{h}\left(\beta_{0}, u\right)=\frac{s_{h}^{(2)}\left(\beta_{0}, u\right)}{s_{h}^{(0)}\left(\beta_{0}, u\right)}-e_{h}\left(\beta_{0}, u\right)^{2}, \quad \text { em que } \\
& e_{h}\left(\beta_{0}, u\right)=\frac{s_{h}^{(1)}\left(\beta_{0}, u\right)}{s_{h}^{(0)}\left(\beta_{0}, u\right)} \mathrm{e}
\end{aligned}
$$

$v_{h}\left(\beta_{0}, u\right) s_{h}^{(0)}\left(\beta_{0}, u\right) \alpha_{0 h}(u)>L$, uniformemente em $u \in[0, \tau]$ e para toda transição do tipo $h(h=1, \ldots, H-1)$.

\section{Suposição D (Viés)}

1. $\beta_{0}(u)$ é Lipshitz'] de ordem $1 \mathrm{em}[0, \tau]$.

2. $\beta_{0}(u)$ tem a segunda derivada limitada uniformemente em $u \in[0, \tau]$.

3. $v_{h}\left(\beta_{0}, u\right) s_{h}^{(0)}\left(\beta_{0}, u\right) \alpha_{0 h}(u)$ é Lipshitz de ordem 1 em $[0, \tau]$ e para toda transição do tipo $h$.

\footnotetext{
${ }^{1}$ Uma função real $f(x)$, definida e contínua em um conjunto aberto $D \subset \mathbb{R}^{2}$ é dita ser Lipshitz com respeito a $x$ para alguma constante $k>0$ se para todos os pontos $x_{1}$ e $x_{2},\left|f\left(x_{1}\right)-f\left(x_{2}\right)\right| \leq k\left|x_{1}-x_{2}\right| \forall x, y$.
} 
Observações: As condições acima são similares àqueles apresentados em Andersen \& Gill (1982) em seus seus estudos sobre coeficientes de regressão.

As Suposições A(1) e A(2) garantem que a quantidade $S^{(i)}$ converge para o valor $s^{(i)}$ enquanto a Suposição A(3) indica a limitação da terceira derivada da função de log-verossimilhança. A Suposição B é uma adaptação da condição de Lindeberg para a o caso multi-estados, para mostrar a convergência fraca.

\subsubsection{Consistência}

Para provar a consistência do estimador de máxima verossimilhança, aproximamos a função de log-verossimilhança pela expansão de Taylor em torno do verdadeiro valor do parâmetro, $\beta_{0}$, e então, usamos o teorema do ponto fixo como em Aitchison \& Silvey (1958).

Teorema 3.1. Suponha que

(a) $\lim _{n} n\|l\|^{10}=0 \quad$ (viés $\left.\rightarrow 0\right)$,

(b) $\lim _{n} n\|l\|^{4}=\infty \quad$ (variância converge),

(c) Suposições A, C, D(1), limsup $\sup _{n} \frac{l_{(K)}}{l_{(1)}}<\infty$;

então, para $\widehat{\beta}^{n}$ que maximiza $\mathcal{L}_{n}(\beta)$ em $\Theta_{K}$,

$$
\int_{0}^{\tau}\left[\widehat{\beta}^{n}(u)-\beta_{0}(u)\right]^{2} d u=O_{p}\left(\left(n\|l\|^{2}\right)^{-1}\right)+O\left(\|l\|^{4}\right),
$$

quando $n \rightarrow \infty$.

\section{Demonstração}

A demonstração segue de maneira similar àquela apresentada em Murphy \& Sen (1991), mas adaptado para o caso multi-estados. Inicialmente reescrevemos a expressão

$$
\int_{0}^{\tau}\left(\widehat{\beta}^{n}(u)-\beta_{0}\right)^{2} \mathrm{~d} u
$$


por meio de uma desigualdade,

$$
\begin{aligned}
\int_{0}^{\tau}\left[\widehat{\beta}^{n}(u)-\beta_{0}(u)\right]^{2} \mathrm{~d} u \leq & 2 \int_{0}^{\tau}\left[\widehat{\beta}^{n}(u)-\bar{\beta}^{n}(u)\right]^{2} \mathrm{~d} u \\
& +2 \int_{0}^{\tau}\left[\bar{\beta}^{n}(u)-\beta_{0}(u)\right]^{2} \mathrm{~d} u
\end{aligned}
$$

Usando a definição de $\bar{\beta}^{n}(u)$, suposição $\mathrm{D}(1)$ e $\lim \sup _{n}\left(l_{(K)} / l_{(1)}\right)<\infty$, mostramos que o segundo termo é $O\left(\|l\|^{4}\right)$, conforme resultado apresentado na Seção B.2.

Mostrar que o primeiro termo é $O_{p}\left(\left(n\|l\|^{2}\right)^{-1}\right)$ é equivalente a mostrar que

$$
n\|l\|^{4}\left\|\widehat{\beta}^{n}-\bar{\beta}^{n}\right\|^{2}=O_{p}(1) .
$$

Para isso, usa-se o resultado do teorema do ponto fixo do Lema 2 de Aitchison \& Silvey (1958). Os Lemas A.1, A.2 e A.3 também são necessários para auxiliar nesta demonstração e são equivalentes àqueles apresentados em Murphy \& Sen (1991).

Considere a quantidade

$$
\begin{gathered}
\delta_{h}^{2}=8 L^{-2}\|l\|^{4} \int_{0}^{\tau} \sum_{k=1}^{K} \int_{0}^{\tau} \mathbb{I}_{k}(u) l_{k}^{-2} v_{h}\left(\beta_{0}, u\right) s_{h}^{(0)}\left(\beta_{0}, u\right) \alpha_{0 h}(u) \mathrm{d} u \\
\operatorname{com} \quad h=1, \ldots, H-1 .
\end{gathered}
$$

O teorema do ponto fixo consiste em mostrar que, se $\exists \beta_{k} \in \Theta_{K}$ tal que

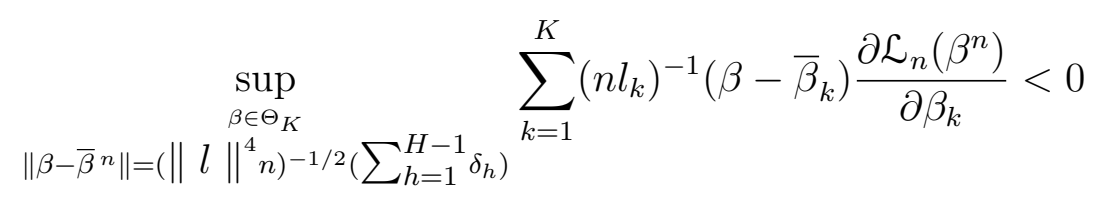

com probabilidade que tende a 1 quando $n \rightarrow \infty$ e $\|l\| \rightarrow 0$, então existe $\widehat{\beta}^{n} \in \Theta_{K}$ tal que

$$
\left.\frac{\partial}{\partial \beta_{k}} \mathcal{L}_{n}(\beta)\right|_{\beta=\widehat{\beta}^{n}}=0 \forall k \quad \text { e } \quad\left\|\widehat{\beta}^{n}-\bar{\beta}^{n}\right\| \leq \frac{\sum_{h=1}^{H-1} \delta_{h}}{\left(n\|l\|^{4}\right)^{1 / 2}} .
$$


Para mostrar que a condição (3.7) é verdadeira, necessitamos dos Lemas A.1, A.2 e A.3.

Reescrevendo a expressão à direita de (3.7) como uma expansão de Taylor em torno de $\bar{\beta}_{k}^{n}$, temos

$$
\begin{aligned}
& \sum_{k=1}^{K} \frac{1}{n l_{k}} \frac{\partial}{\partial \beta_{k}} \mathcal{L}_{n}(\beta)\left[\beta_{k}-\bar{\beta}_{k}^{n}\right] \leq \\
\leq & \sum_{k=1}^{K} \sum_{h=1}^{H-1} \frac{1}{n l_{k}}\left[\frac{\partial}{\partial \beta_{k}} \mathcal{L}_{n h}\left(\bar{\beta}_{k}^{n}\right)\right]\left[\beta_{k}-\bar{\beta}_{k}^{n}\right] \\
& +\sum_{k=1}^{K} \sum_{h=1}^{H-1} \frac{1}{n l_{k}}\left[\frac{\partial^{2}}{\partial \beta_{k}^{2}} \mathcal{L}_{n h}\left(\bar{\beta}_{k}^{n}\right)\right]\left[\beta_{k}-\bar{\beta}_{k}^{n}\right]^{2} \\
& +\frac{1}{2} \sum_{k=1}^{K} \sum_{h=1}^{H-1} \frac{1}{n l_{k}}\left[\frac{\partial^{3}}{\partial \beta_{k}^{3}} \mathcal{L}_{n h}\left(\beta^{*}\right)\right]\left[\beta_{k}-\bar{\beta}_{k}^{n}\right]^{3},
\end{aligned}
$$

em que $\left\|\beta^{*}-\bar{\beta}^{n}\right\| \leq\left\|\beta-\bar{\beta}^{n}\right\|$.

Note que o termo (3.9) pode ser escrito como

$$
\begin{aligned}
& \sum_{h=1}^{H-1} \sum_{k=1}^{K} \frac{1}{n l_{k}}\left[\frac{\partial}{\partial \beta_{k}} \mathcal{L}_{n h}\left(\bar{\beta}_{k}^{n}\right)\right]\left[\beta_{k}-\bar{\beta}_{k}^{n}\right] \\
= & \sum_{k=1}^{K} \frac{1}{n l_{k}}\left[\frac{\partial}{\partial \beta_{k}} \mathcal{L}_{n 1}\left(\bar{\beta}_{k}^{n}\right)\right]\left[\beta_{k}-\bar{\beta}_{k}^{n}\right]+\ldots+\sum_{k=1}^{K} \frac{1}{n l_{k}}\left[\frac{\partial}{\partial \beta_{k}} \mathcal{L}_{n(H-1)}\left(\bar{\beta}_{k}^{n}\right)\right]\left[\beta_{k}-\bar{\beta}_{k}^{n}\right] \\
\leq & {\left[\sum_{k=1}^{K}\left(\frac{1}{n l_{k}} \frac{\partial}{\partial \beta_{k}} \mathcal{L}_{n 1}\left(\bar{\beta}_{k}^{n}\right)\right)^{2}\right]^{1 / 2}\left[\sum_{k=1}^{K}\left[\beta_{k}-\bar{\beta}_{k}^{n}\right]^{2}\right]^{1 / 2}+\ldots+} \\
& +\left[\sum_{k=1}^{K}\left(\frac{1}{n l_{k}} \frac{\partial}{\partial \beta_{k}} \mathcal{L}_{n(H-1)}\left(\bar{\beta}_{k}^{n}\right)\right)^{2}\right]^{1 / 2}\left[\sum_{k=1}^{K}\left[\beta_{k}-\bar{\beta}_{k}^{n}\right]^{2}\right]^{1 / 2} \\
\leq & \sum_{h=1}^{H-1}\left[\sum_{k=1}^{K}\left(\frac{1}{n l_{k}}\right)^{2}\left|\frac{\partial}{\partial \beta_{k}} \mathcal{L}_{n h}\left(\bar{\beta}_{k}^{n}\right)\right|^{2}\right]^{1 / 2}\left\|\beta-\bar{\beta}^{n}\right\|,
\end{aligned}
$$


e pelo item A.3 do Lema A.2, temos

$$
\begin{aligned}
= & \sum_{h=1}^{H-1}\left\{2\left(\|l\|^{4} n\right)^{-1}\left[\|l\|^{4} \int_{0}^{\tau} \sum_{k=1}^{K} \mathbb{I}_{k}(u) l_{k}^{-2} v_{h}\left(\beta_{0}, u\right) s_{h}^{(0)}\left(\beta_{0}, u\right) \alpha_{0 h}(u) \mathrm{d} u+o_{p}(1)\right]\right. \\
& \left.+O_{p}\left(n^{-1}\right)+O_{p}\left(\|l\|^{6}\right)\right\}^{1 / 2}\left\|\beta-\bar{\beta}^{n}\right\| \\
= & \sum_{h=1}^{H-1}\left\{\left(\|l\|^{4} n\right)^{-1}\left[\frac{L^{2} \delta_{h}^{2}}{4}+o_{p}(1)\right]+O_{p}\left(n^{-1}\right)+O_{p}\left(\|l\|^{6}\right)\right\}^{1 / 2}\left\|\beta-\bar{\beta}^{n}\right\|,
\end{aligned}
$$

e pela definição de $\delta_{h}$ dada em $\sqrt[3.6]{ }$ e sob a condição $\left\|\beta-\bar{\beta}^{n}\right\|^{2}=\frac{1}{n\|l\|^{4}}\left[\sum_{h=1}^{H-1} \delta_{h}\right]^{2}$,

$$
\begin{aligned}
= & \sum_{h=1}^{H-1}\left\{\frac{\left(\|l\|^{4} n\right)^{-1}\left[\frac{L^{2} \delta_{h}^{2}}{4}+o_{p}(1)\right]+O_{p}\left(n^{-1}\right)+O_{p}\left(\|l\|^{6}\right)}{\left\|\beta-\bar{\beta}^{n}\right\|^{2}}\right\}^{1 / 2}\left\|\beta-\bar{\beta}^{n}\right\|^{2} \\
= & \sum_{h=1}^{H-1}\left\{\frac{\left(\|l\|^{4} n\right)^{-1}\left[\frac{L^{2} \delta_{h}^{2}}{4}+o_{p}(1)\right]+O_{p}\left(n^{-1}\right)+O_{p}\left(\|l\|^{6}\right)}{\frac{1}{n\|l\|^{4}}\left[\sum_{h=1}^{H-1} \delta_{h}\right]^{2}}\right\}\left\|\beta-\bar{\beta}^{n}\right\|^{2} \\
= & \sum_{h=1}^{H-1}\left\{\left[\frac{L^{2} \delta_{h}^{2}}{4\left[\sum_{h=1}^{H-1} \delta_{h}\right]^{2}}+o_{p}\left(\frac{1}{\left[\sum_{h=1}^{H-1} \delta_{h}\right]^{2}}\right)\right]\right. \\
& \left.+O_{p}\left(\frac{\|l\|^{4}}{\left[\sum_{h=1}^{H-1} \delta_{h}\right]^{2}}\right)+O_{p}\left(\frac{n\|l\|^{10}}{\left[\sum_{h=1}^{H-1} \delta_{h}\right]^{2}}\right)\right\}^{1 / 2}\left\|\beta-\bar{\beta}^{n}\right\|^{2} \\
\approx & \frac{L}{2} \frac{\sum_{h=1}^{H-1} \sqrt{\delta_{h}^{2}}\left\|\beta-\bar{\beta}^{n}\right\|^{2}=\frac{L}{2}\left\|\beta-\bar{\beta}^{n}\right\|^{2} .}{\sum_{h=1}^{H-1} \delta_{h}} \| \beta^{2}
\end{aligned}
$$

Com relação ao termo 3.10 , somando e subtraindo a quantidade $\sum_{h=1}^{H-1} \sum_{k=1}^{K} l_{k}^{-1} \sigma_{k h}^{2}\left[\beta_{k}-\bar{\beta}_{k}^{n}\right]^{2}$ 
e usando o resultado do Lema A.2, temos

$$
\begin{aligned}
& \sum_{h=1}^{H-1} \sum_{k=1}^{K} \frac{1}{n l_{k}}\left[\frac{\partial^{2}}{\partial \beta_{k}^{2}} \mathcal{L}_{n h}\left(\bar{\beta}^{n}\right)\right]\left[\beta_{k}-\bar{\beta}_{k}^{n}\right]^{2}+\sum_{h=1}^{H-1} \sum_{k=1}^{K} l_{k}^{-1} \sigma_{k h}^{2}\left[\beta_{k}-\bar{\beta}_{k}^{n}\right]^{2} \\
&-\sum_{h=1}^{H-1} \sum_{k=1}^{K} l_{k}^{-1} \sigma_{k h}^{2}\left[\beta_{k}-\bar{\beta}_{k}^{n}\right]^{2} \\
&= \sum_{h=1}^{H-1} \sum_{k=1}^{K}\left[\frac{1}{n l_{k}} \frac{\partial^{2}}{\partial \beta_{k}^{2}} \mathcal{L}_{n h}\left(\bar{\beta}^{n}\right)+l_{k}^{-1} \sigma_{k h}^{2}\right]\left[\beta_{k}-\bar{\beta}_{k}^{n}\right]^{2} \\
&-\sum_{h=1}^{H-1} \sum_{k=1}^{K} l_{k}^{-1}\left[\int_{0}^{\tau} \mathbb{I}_{k}(u) v_{h}\left(\beta_{0}, u\right) s_{h}^{(0)}\left(\beta_{0}, u\right) \alpha_{0 h}(u) \mathrm{d} u\right]\left[\beta_{k}-\bar{\beta}_{k}^{n}\right]^{2} \\
& \leq \sum_{h=1}^{H-1} \sum_{k=1}^{K}\left[\frac{1}{n l_{k}} \frac{\partial^{2}}{\partial \beta_{k}^{2}} \mathcal{L}_{n h}\left(\bar{\beta}^{n}\right)+l_{k}^{-1} \sigma_{k h}^{2}\right]\left[\beta_{k}-\bar{\beta}_{k}^{n}\right]^{2} \\
&-(H-1) L \sum_{k=1}^{K} l_{k}^{-1}\left(\int_{0}^{\tau} \mathbb{I}_{k}(u) \mathrm{d} u\right)\left[\beta_{k}-\bar{\beta}_{k}^{n}\right]^{2} \\
&= \sum_{h=1}^{H-1} \max _{1 \leq k \leq K}\left|\frac{1}{n l_{k}} \frac{\partial^{2}}{\partial \beta_{k}^{2}} \mathcal{L}_{n h}\left(\bar{\beta}^{n}\right)+l_{k}^{-1} \sigma_{k h}^{2}\right| \sum_{k=1}^{K}\left[\beta_{k}-\bar{\beta}_{k}^{n}\right]^{2}-(H-1) L \sum_{k=1}^{K}\left(l_{k}\right) l_{k}^{-1}\left[\beta_{k}-\bar{\beta}_{k}^{n}\right]^{2} \\
&= \sum_{h=1}^{H-1} \max _{1 \leq k \leq K}\left|\frac{1}{n l_{k}} \frac{\partial^{2}}{\partial \beta_{k}^{2}} \mathcal{L}_{n h}\left(\bar{\beta}^{n}\right)+l_{k}^{-1} \sigma_{k h}^{2}\right|\left\|\beta-\bar{\beta}^{n}\right\|^{2}-(H-1) L \sum_{k=1}^{K}\left[\beta_{k}-\bar{\beta}_{k}^{n}\right]^{2} \\
&= \sum_{h=1}^{H-1} \max _{1 \leq k \leq K}\left|\frac{1}{n l_{k}} \frac{\partial^{2}}{\partial \beta_{k}^{2}} \mathcal{L}_{n h}\left(\bar{\beta}^{n}\right)+l_{k}^{-1} \sigma_{k h}^{2}\right|\left\|\beta-\bar{\beta}^{n}\right\|^{2}-(H-1) L\left\|\beta-\bar{\beta}^{n}\right\|^{2} \\
&=(H-1)\left\{O_{p}\left(\left(\sqrt{n}\left\|_{n}\right\| \|^{2}\right)^{-1}\right)+o_{p}(1)\right\}\left\|\beta-\bar{\beta}^{n}\right\|^{2}-(H-1) L\left\|\beta-\bar{\beta}^{n}\right\|^{2} \\
&=(H-1)\left\{O_{p}\left(\left(\sqrt{n}\left\|_{n}\right\| \|^{2}\right)^{-1}\right)+o_{p}(1)-L\right\}\left\|\beta-\bar{\beta}^{n}\right\|^{2} . \\
&=
\end{aligned}
$$


Usando o resultado A.5 do Lema A.2, o termo 3.11 pode ser escrito como

$$
\begin{aligned}
& \frac{1}{2} \sum_{k=1}^{K} \sum_{h=1}^{H-1} \frac{1}{n l_{k}}\left[\frac{\partial^{3}}{\partial \beta_{k}^{3}} \mathcal{L}_{n h}\left(\beta^{*}\right)\right]\left[\beta_{k}-\bar{\beta}_{k}^{n}\right]^{3} \\
\leq & \sum_{h=1}^{H-1} \max _{1 \leq k \leq K} \sup _{\| \beta^{*} \Theta_{K}} \frac{1}{n l_{k}}\left|\frac{\partial^{3}}{\partial \beta_{k}^{3}} \mathcal{L}_{n h}\left(\beta^{*}\right)\right| \sum_{k=1}^{K}\left[\beta_{k}-\bar{\beta}_{k}^{n}\right]^{3} \\
\leq & \sum_{h=1}^{H-1}\left[O_{p}\left(\left(\sqrt{n}\|l\|^{2}\right)^{-1}\right)+O_{p}(1)\right]\left\|\beta-\bar{\beta}^{n}\right\|^{3} \\
= & (H-1)\left[O_{p}\left(\left(\sqrt{n}\|l\|^{2}\right)^{-1}\right)+O_{p}(1)\right]\left\|\beta-\bar{\beta}^{n}\right\|^{3} .
\end{aligned}
$$

Substituindo os resultados (3.12), (3.13) e (3.14) nas expressões (3.9), 3.10) e (3.11), respectivamente, encontramos

$$
\begin{aligned}
& \sup _{\operatorname{se\Theta }_{K}} \sum_{k=1}^{K} \frac{1}{n l} \frac{\partial}{\partial \beta_{k}} \mathcal{L}_{n}\left(\beta^{n}\right)\left[\beta_{k}-\bar{\beta}_{k}^{n}\right] \\
& \left\|\beta-\bar{\beta}^{n}\right\|=\left(\|l\|^{4} n\right)^{-1 / 2}\left(\sum_{h=1}^{H-1} \delta_{h}\right) \\
& =\frac{L}{2}\left\|\beta-\bar{\beta}^{n}\right\|^{2}+(H-1)\left\{O_{p}\left(\left(\sqrt{n}\|l\|^{2}\right)^{-1}\right)+o_{p}(1)-L\right\}\left\|\beta-\bar{\beta}^{n}\right\|^{2} \\
& \quad+\left[O_{p}\left(\left(\sqrt{n}\|l\|^{2}\right)^{-1}\right)+O_{p}(1)\right]\left\|\beta-\bar{\beta}^{n}\right\|^{3} \\
& =\frac{L}{2}\left\|\beta-\bar{\beta}^{n}\right\|^{2} \\
& \quad+\left[O_{p}\left(\left(\sqrt{n}\|l\|^{2}\right)^{-1}\right)+o_{p}(1)-(H-1) L+\left(O_{p}\left(\left(\sqrt{n}\|l\|^{2}\right)^{-1}\right)+O_{p}(1)\right)\left\|\beta-\bar{\beta}^{n}\right\|\right]\left\|\beta-\bar{\beta}^{n}\right\|^{2} \\
& \approx[L / 2-(H-1) L]\left\|\beta-\bar{\beta}^{n}\right\|^{2}<0 .
\end{aligned}
$$

Assim, podemos aplicar o Lema 2 de Aitchison \& Silvey (1958) e concluir que a probabilidade de

$$
\sup _{\substack{\beta \in \Theta_{K} \\\left\|\beta-\bar{\beta}^{n}\right\|=\left(\|l\|^{4} n\right)^{-1 / 2}\left(\sum_{h=1}^{H-1} \delta_{h}\right)}} \sum_{k=1}^{K}\left(n l_{k}\right)^{-1} \frac{\partial}{\partial \beta_{k}} \mathcal{L}_{n}\left(\beta^{n}\right)\left(\beta_{k}-\bar{\beta}_{k}\right)<1
$$

converge para 1. 
Observação: A idéia das suposições $(a)$ e $(b)$ é que a partição escolhida deve ser de tal modo que quando aumentamos o tamanho amostral, devemos ter $\lim _{n} n\|l\|^{10}=0 \mathrm{e}$ $\lim _{n} n\|l\|^{4}=\infty$. A suposição $(c)$ garante que o comprimento do maior intervalo não seja infinitamente maior que o comprimento do menor intervalo.

\subsubsection{Convergência em distribuição}

McKeague (1988) e Pedroso de Lima \& Sen (1999) consideram o estimador integrado para fazer inferência. Dessa forma, consegue-se melhores taxas de convergência. Assim, a convergência fraca é mostrada como um processo integrado, envolvendo um termo que converge para um processo gaussiano e outro que converge para zero em probabilidade. O resultado é dado pelo teorema a seguir.

Teorema 3.2. Suponha que as condições $A, B, C$ e D acontecem juntamente com

(a) $\lim _{n} n\|l\|^{8}=0$ (Viés $\left.\rightarrow 0\right)$,

(b) $\lim _{n} n\|l\|^{4}=\infty$ (Variância converge),

(c) $\lim \sup _{n} \frac{l_{(K)}}{l_{(1)}}<\infty$;

então, o processo integrado

$$
\sqrt{n} \int_{0}^{t \wedge r}\left(\widehat{\beta}^{n}(u)-\beta_{0}(u)\right) d u \stackrel{q . c .}{\Rightarrow} G
$$

em que $G$ é um martingal gaussiano multivariado, tal que $G(0)=0 e$

$$
\operatorname{cov}(G(t), G(r))=\int_{0}^{t \wedge r}\left(\sum_{h=1}^{H-1} v_{h}\left(\beta_{0}, u\right) s_{h}^{(0)}\left(\beta_{0}, u\right) \alpha_{0 h}(u)\right)^{-1} d u, \quad r, t \in[0, \tau] .
$$

\section{Demonstração}


Note que

$$
\sqrt{n} \int_{0}^{t}\left(\widehat{\beta}^{n}(u)-\beta_{0}(u)\right) \mathrm{d} u=\sqrt{n} \int_{0}^{t}\left(\widehat{\beta}^{n}(u)-\bar{\beta}^{n}(u)\right) \mathrm{d} u+\sqrt{n} \int_{0}^{t}\left(\bar{\beta}^{n}(u)-\beta_{0}(u)\right) \mathrm{d} u \cdot(3.1
$$

Usando a Suposição D, Murphy \& Sen (1991) provam que

$$
\sup _{t \in[0, \tau]}\left|\sqrt{n} \int_{0}^{t}\left(\bar{\beta}^{n}(u)-\beta_{0}(u)\right) \mathrm{d} u\right|=O\left(n^{1 / 2}\|l\|^{4}\right) .
$$

Para mostrar que o primeiro termo, $\sqrt{n} \int_{0}^{t}\left(\widehat{\beta}^{n}(u)-\bar{\beta}^{n}(u)\right) \mathrm{d} u \stackrel{\text { q.c. }}{\Rightarrow} G$, seguimos os mesmos passos de Murphy \& Sen (1991).

Note que $\frac{1}{\sqrt{n}} \frac{\partial}{\partial \beta_{k}} \mathcal{L}_{n}\left(\widehat{\beta}^{n}\right)=0$. Então,

$$
\frac{1}{\sqrt{n}} \frac{\partial}{\partial \beta_{k}} \mathcal{L}_{n}\left(\bar{\beta}^{n}\right)+\sqrt{n}\left(\widehat{\beta}_{k}^{n}-\bar{\beta}_{k}^{n}\right)\left[\frac{1}{n} \frac{\partial^{2}}{\partial \beta_{k}^{2}} \mathcal{L}_{n}\left(\bar{\beta}^{n}\right)+\frac{1}{2 n} \frac{\partial^{3}}{\partial \beta_{k}^{3}} \mathcal{L}_{n}\left(\beta^{*}\right)\left(\widehat{\beta}_{k}^{n}-\bar{\beta}_{k}^{n}\right)\right]=0
$$

em que $\left\|\beta^{*}-\widehat{\beta}^{n}\right\| \leq\left\|\bar{\beta}^{n}-\widehat{\beta}^{n}\right\|$.

Defina

$$
\widehat{\sigma}_{k h}^{2}=-\frac{1}{n} \frac{\partial^{2}}{\partial \beta_{k}^{2}} \mathcal{L}_{n h}\left(\bar{\beta}^{n}\right)-\frac{1}{2 n} \frac{\partial^{3}}{\partial \beta_{k}^{3}} \mathcal{L}_{n h}\left(\beta^{*}\right)\left(\widehat{\beta}_{k}^{n}-\bar{\beta}_{k}^{n}\right) .
$$

Então, 3.16 pode ser reescrita como

$$
\frac{1}{\sqrt{n}} \frac{\partial}{\partial \beta_{k}} \mathcal{L}_{n}\left(\bar{\beta}^{n}\right)-\sqrt{n}\left(\widehat{\beta}_{k}^{n}-\bar{\beta}_{k}^{n}\right) \sum_{h=1}^{H-1} \widehat{\sigma}_{k h}^{2}=0 .
$$


Assim,

$$
\begin{aligned}
\sqrt{n}\left(\widehat{\beta}_{k}^{n}-\bar{\beta}_{k}^{n}\right)= & \frac{1}{\sqrt{n}} \frac{\partial}{\partial \beta_{k}} \mathcal{L}_{n}\left(\bar{\beta}^{n}\right) \frac{1}{\sum_{h=1}^{H-1} \widehat{\sigma}_{k h}^{2}} \\
= & \frac{1}{\sqrt{n}} \sum_{h=1}^{H-1} \frac{\partial}{\partial \beta_{k}} \mathcal{L}_{n h}\left(\bar{\beta}^{n}\right) \frac{1}{\sum_{h=1}^{H-1} \widehat{\sigma}_{k h}^{2}}-\left(\frac{1}{\sqrt{n}} \sum_{h=1}^{H-1} \frac{\partial}{\partial \beta_{k}} \mathcal{L}_{n h}\left(\bar{\beta}^{n}\right) \frac{1}{\sum_{h=1}^{H-1} \sigma_{k h}^{2}}\right) \\
& +\left(\frac{1}{\sqrt{n}} \sum_{h=1}^{H-1} \frac{\partial}{\partial \beta_{k}} \mathcal{L}_{n h}\left(\bar{\beta}^{n}\right) \frac{1}{\sum_{h=1}^{H-1} \sigma_{k h}^{2}}\right) \\
= & \frac{1}{\sqrt{n}}\left[\frac{1}{\sum_{h=1}^{H-1} \widehat{\sigma}_{k h}^{2}}-\frac{1}{\sum_{h=1}^{H-1} \sigma_{k h}^{2}}\right] \sum_{h=1}^{H-1} \frac{\partial}{\partial \beta_{k}} \mathcal{L}_{n h}\left(\bar{\beta}^{n}\right) \\
& +\frac{1}{\sqrt{n}} \sum_{h=1}^{H-1} \frac{\partial}{\partial \beta_{k}} \mathcal{L}_{n h}\left(\bar{\beta}^{n}\right) \frac{1}{\sum_{h=1}^{H-1} \sigma_{k h}^{2}} .
\end{aligned}
$$

O Lema A.3 tem como conseqüência que $P\left[\min _{1 \leq k \leq K} l_{k}^{-1} \sum_{h=1}^{H-1} \widehat{\sigma}_{k h}^{2}>(1 / 2) L\right] \rightarrow 1$. Então, vamos considerar a quantidade $\sqrt{n} \int_{0}^{t}\left(\widehat{\beta}^{n}(u)-\bar{\beta}^{n}(u)\right) \mathrm{d} u$ apenas dentro desse conjunto. Portanto, multiplicando $\sqrt{n}\left(\widehat{\beta}^{n}(u)-\bar{\beta}^{n}(u)\right)$ por $\mathbb{I}_{k}(u)$ e integrando de 0 a $t$, temos

$$
\begin{aligned}
& \sqrt{n} \int_{0}^{t} \sum_{k=1}^{K} \mathbb{I}_{k}(u)\left(\widehat{\beta}^{n}(u)-\bar{\beta}^{n}(u)\right) \mathrm{d} u \\
= & \frac{1}{\sqrt{n}} \int_{0}^{t} \sum_{k=1}^{K} \mathbb{I}_{k}(u)\left[\frac{1}{\sum_{h=1}^{H-1} \widehat{\sigma}_{k h}^{2}}-\frac{1}{\sum_{h=1}^{H-1} \sigma_{k h}^{2}}\right] \sum_{h=1}^{H-1} \frac{\partial}{\partial \beta_{k}} \mathcal{L}_{n h}\left(\bar{\beta}^{n}\right) \mathrm{d} u \\
& +\frac{1}{\sqrt{n}} \int_{0}^{t} \sum_{k=1}^{K} \mathbb{I}_{k}(u) \frac{1}{\sum_{h=1}^{H-1} \sigma_{k h}^{2}} \sum_{h=1}^{H-1} \frac{\partial}{\partial \beta_{k}} \mathcal{L}_{n h}\left(\bar{\beta}^{n}\right) \mathrm{d} u .
\end{aligned}
$$


Para mostrar que (3.17) é $o_{p}(1)$, note que seu supremo é

$$
\begin{aligned}
& \frac{1}{\sqrt{n}} \int_{0}^{\tau} \sum_{k=1}^{K} \mathbb{I}_{k}(u)\left|\left[\frac{1}{\sum_{h=1}^{H-1} \widehat{\sigma}_{k h}^{2}}-\frac{1}{\sum_{h=1}^{H-1} \sigma_{k h}^{2}}\right] \sum_{h=1}^{H-1} \frac{\partial}{\partial \beta_{k}} \mathcal{L}_{n h}\left(\bar{\beta}^{n}\right)\right| \mathrm{d} u \\
& \leq \sum_{h=1}^{H-1} \frac{1}{\sqrt{n}} \int_{0}^{\tau} \sum_{k=1}^{K} \mathbb{I}_{k}(u)\left|\left[\frac{1}{\sum_{h=1}^{H-1} \widehat{\sigma}_{k h}^{2}}-\frac{1}{\sum_{h=1}^{H-1} \sigma_{k h}^{2}}\right] \frac{\partial}{\partial \beta_{k}} \mathcal{L}_{n h}\left(\bar{\beta}^{n}\right)\right| \mathrm{d} u=\sum_{h=1}^{H-1} g_{h} .
\end{aligned}
$$

Além disso, o quadrado de cada uma das parcelas acima é $o_{p}(1)$, conforme mostrado a seguir.

$$
\begin{aligned}
& g_{h}^{2}=\frac{1}{n}\left[\int_{0}^{\tau} \sum_{k=1}^{K} \mathbb{I}_{k}(u)\left|\left[\frac{1}{\sum_{h=1}^{H-1} \widehat{\sigma}_{k h}^{2}}-\frac{1}{\sum_{h=1}^{H-1} \sigma_{k h}^{2}}\right] \frac{\partial}{\partial \beta_{k}} \mathcal{L}_{n h}\left(\bar{\beta}^{n}\right)\right| \mathrm{d} u\right]^{2} \\
& \leq \frac{1}{n} \sum_{k=1}^{K} l_{k}^{2}\left[\frac{1}{\sum_{h=1}^{H-1} \widehat{\sigma}_{k h}^{2}}-\frac{1}{\sum_{h=1}^{H-1} \sigma_{k h}^{2}}\right]^{2} \sum_{k=1}^{K}\left[\frac{\partial}{\partial \beta_{k}} \mathcal{L}_{n h}\left(\bar{\beta}^{n}\right)\right]^{2} \\
& =\frac{1}{n} \sum_{k=1}^{K} \frac{l_{k}^{2}}{l_{k}^{2}} l_{k}^{2}\left[\frac{1}{\sum_{h=1}^{H-1} \widehat{\sigma}_{k h}^{2}}-\frac{1}{\sum_{h=1}^{H-1} \sigma_{k h}^{2}}\right]^{2} \sum_{k=1}^{K} \frac{n l_{k}^{2}}{n l_{k}^{2}}\left[\frac{\partial}{\partial \beta_{k}} \mathcal{L}_{n h}\left(\bar{\beta}^{n}\right)\right]^{2} \\
& \leq l_{(1)}^{-2} \sum_{k=1}^{K}\left[\frac{l_{k}^{2}}{\sum_{h=1}^{H-1} \widehat{\sigma}_{k h}^{2}}-\frac{l_{k}^{2}}{\sum_{h=1}^{H-1} \sigma_{k h}^{2}}\right]^{2} n l_{(K)}^{2} \sum_{k=1}^{K}\left(n l_{k}\right)^{-2}\left[\frac{\partial}{\partial \beta_{k}} \mathcal{L}_{n h}\left(\bar{\beta}^{n}\right)\right]^{2} \\
& \leq \sum_{k=1}^{K}\left[\frac{l_{k}^{2}}{\sum_{h=1}^{H-1} \widehat{\sigma}_{k h}^{2}}-\frac{l_{k}^{2}}{\sum_{h=1}^{H-1} \sigma_{k h}^{2}}\right]^{2} n \frac{l_{(K)}^{2}}{l_{(1)}^{2}} \sum_{k=1}^{K}\left(n l_{k}\right)^{-2}\left[\frac{\partial}{\partial \beta_{k}} \mathcal{L}_{n h}\left(\bar{\beta}^{n}\right)\right]^{2} \\
& \leq \sum_{k=1}^{K} \frac{l_{k}^{2}}{\sum_{h=1}^{H-1} \widehat{\sigma}_{k h}^{2}} \frac{l_{k}^{2}}{\sum_{h=1}^{H-1} \sigma_{k h}^{2}}\left[\sum_{h=1}^{H-1} \sigma_{k h}^{2}-\sum_{h=1}^{H-1} \widehat{\sigma}_{k h}^{2}\right]^{2} n \frac{l_{(K)}^{2}}{l_{(1)}^{2}} \sum_{k=1}^{K}\left(n l_{k}\right)^{-2}\left[\frac{\partial}{\partial \beta_{k}} \mathcal{L}_{n h}\left(\bar{\beta}^{n}\right)\right]^{2} \\
& \leq O_{p}(1)\left[\sum_{h=1}^{H-1} \sigma_{k h}^{2}-\sum_{h=1}^{H-1} \widehat{\sigma}_{k h}^{2}\right]^{2} n \frac{l_{(K)}^{2}}{l_{(1)}^{2}} \sum_{k=1}^{K}\left(n l_{k}\right)^{-2}\left[\frac{\partial}{\partial \beta_{k}} \mathcal{L}_{n h}\left(\bar{\beta}^{n}\right)\right]^{2} \\
& =O_{p}(1)\left[\|l\|^{4}\left(O_{p}\left[\frac{1}{n\|l\|^{4}}\right]+O_{p}\left(\|l\|^{2}\right)+O_{p}\left(\left\|\widehat{\beta}^{n}-\bar{\beta}^{n}\right\|\right)\right)\right] n \\
& \cdot\left[O_{p}\left(\frac{1}{n\|l\|^{4}}\right)+o_{p}(1)+O_{p}\left(\|l\|^{6}\right)+O_{p}\left(\frac{1}{n}\right)\right] \\
& =o_{p}(1) \text {, }
\end{aligned}
$$


dos Lemas A.3 e A.2.

Assim, $g_{h}=o_{p}(1)$. Logo, $\sum_{h=1}^{H-1} g_{h}=o_{p}(1)$ também.

Quanto ao termo (3.18),

$$
\begin{aligned}
& \frac{1}{\sqrt{n}} \int_{0}^{t} \sum_{k=1}^{K} \mathbb{I}_{k}(u) \frac{1}{\sum_{h=1}^{H-1} \sigma_{k h}^{2}} \sum_{h=1}^{H-1} \frac{\partial}{\partial \beta_{k}} \mathcal{L}_{n h}\left(\bar{\beta}^{n}\right) \mathrm{d} u \\
= & \frac{1}{\sqrt{n}} \int_{0}^{t} \sum_{k=1}^{K} \mathbb{I}_{k}(u) \frac{1}{\sum_{h=1}^{H-1} \sigma_{k h}^{2}} \sum_{h=1}^{H-1} \sum_{j=1}^{n} \int_{0}^{\tau} \mathbb{I}_{k}(z)\left[X_{j}(z)-\mathbb{E}_{h}\left(\bar{\beta}^{n}, z\right)\right] \mathrm{d} N_{h j}(z) \mathrm{d} u .
\end{aligned}
$$

A idéia é usar o Lema 4.1 de McKeague (1988) para demonstrar que a expressão acima converge fracamente para $G$. Seja $Z(t)=\sum_{h=1}^{H-1} Z_{h}(t)$, em que

$$
Z_{h}(t)=\frac{1}{\sqrt{n}} \sum_{j=1}^{n} \int_{0}^{t}\left[\sum_{k=1}^{K} \mathbb{I}_{k}(u) \frac{l_{k}}{\sigma_{k}^{2}}\right]\left[X_{j}(u)-\mathbb{E}_{h}\left(\bar{\beta}^{n}, u\right)\right] \mathrm{d} N_{h j}(u) .
$$

Usando a decomposição Doob-Meyer [(Andersen et al., 1993, pág.66 )] em $\sum_{h=1}^{H-1} Z_{h}(t)$, temos

$$
\begin{aligned}
\sum_{h=1}^{H-1} Z_{h}(t)= & \frac{1}{\sqrt{n}} \sum_{j=1}^{n} \int_{0}^{t}\left[\sum_{k=1}^{K} \mathbb{I}_{k}(u) \frac{l_{k}}{\sum_{h=1}^{H-1} \sigma_{k h}^{2}}\right] \sum_{h=1}^{H-1}\left[X_{j}(u)-\mathbb{E}_{h}\left(\bar{\beta}^{n}, u\right)\right] \mathrm{d} N_{h j}(u) \\
= & \frac{1}{\sqrt{n}} \sum_{j=1}^{n} \int_{0}^{t}\left[\sum_{k=1}^{K} \mathbb{I}_{k}(u) \frac{l_{k}}{\sum_{h=1}^{H-1} \sigma_{k h}^{2}}\right] \sum_{h=1}^{H-1}\left[X_{j}(u)-\mathbb{E}_{h}\left(\bar{\beta}^{n}, u\right)\right] \mathrm{d} M_{h j}(u) \\
& +\sum_{h=1}^{H-1} \sqrt{n} \int_{0}^{t}\left[\sum_{k=1}^{K} \mathbb{I}_{k}(u) \frac{l_{k}}{\sum_{h=1}^{H-1} \sigma_{k h}^{2}}\right] \\
& \cdot\left[\mathbb{E}_{h}\left(\beta_{0}, u\right)-\mathbb{E}_{h}\left(\bar{\beta}^{n}, u\right)\right] S_{h}^{(0)}\left(\beta_{0}, u\right) \alpha_{0 h}(u) \mathrm{d} u .
\end{aligned}
$$

Pelos resultados obtidos no Lema A.4, o supremo de cada parcela do segundo termo 3.20 é 
$o_{p}(1)$. Para o primeiro termo 3.19), a idéia é usar o Teorema Central do Limite de Rebolledo encontrado, por exemplo, em Andersen \& Gill (1982). Para fazê-lo, é necessário observar duas condições. A primeira é mostrar que $\left\langle\sum_{h=1}^{H-1} Y_{h}(t)\right\rangle$ converge para uma função contínua e a segunda é mostrar que o processo de variação previsível relacionado aos saltos converge para zero, em probabilidade.

Denote o processo de variação previsível de 3.19 por $\left\langle\sum_{h=1}^{H-1} Y_{h}(t)\right\rangle$. Ele é dado por

$$
\begin{aligned}
\left\langle\sum_{h=1}^{H-1} Y_{h}(t)\right\rangle= & \frac{1}{n} \int_{0}^{t}\left[\sum_{k=1}^{K} \mathbb{I}_{k}(u) \frac{l_{k}}{\sum_{h=1}^{H-1} \sigma_{k h}^{2}}\right]^{2} \sum_{h=1}^{H-1} \sum_{j=1}^{n}\left[X_{j}(u)-\mathbb{E}_{h}\left(\bar{\beta}^{n}, u\right)\right]^{2} \mathrm{~d} \Lambda_{h j}(u) \\
= & \frac{1}{n} \int_{0}^{t}\left[\sum_{k=1}^{K} \mathbb{I}_{k}(u) \frac{l_{k}}{\sum_{h=1}^{H-1} \sigma_{k h}^{2}}\right]^{2} \sum_{h=1}^{H-1} \sum_{j=1}^{n}\left[X_{j}(u)-\mathbb{E}_{h}\left(\bar{\beta}^{n}, u\right)\right]^{2} e^{X_{j}(u) \beta_{0}(u)} \alpha_{0 h}(u) \mathrm{d} u \\
= & \int_{0}^{t}\left[\sum_{k=1}^{K} \mathbb{I}_{k}(u) \frac{l_{k}}{\sum_{h=1}^{H-1} \sigma_{k h}^{2}}\right]^{2} \\
& \cdot \sum_{h=1}^{H-1}\left[S_{h}^{(2)}\left(\beta_{0}, u\right)+\mathbb{E}_{h}^{2}\left(\bar{\beta}^{n}, u\right) S_{h}^{(0)}\left(\beta_{0}, u\right)-2 \mathbb{E}\left(\bar{\beta}^{n}, u\right) S_{h}^{(1)}\left(\beta_{0}, u\right)\right] \alpha_{0 h}(u) \mathrm{d} u \\
= & \int_{0}^{t}\left[\sum_{k=1}^{K} \mathbb{I}_{k}(u) \frac{l_{k}}{\sum_{h=1}^{H-1} \sigma_{k h}^{2}}\right]^{2} \\
& \cdot \sum_{h=1}^{H-1}\left[\mathbb{V}_{h}\left(\beta_{0}, u\right) S_{h}^{(0)}\left(\beta_{0}, u\right)+\left[\mathbb{E}_{h}\left(\beta_{0}, u\right)-\mathbb{E}_{h}\left(\bar{\beta}^{n}, u\right)\right]^{2} S_{h}^{(0)}\left(\beta_{0}, u\right)\right]^{2} \alpha_{0 h}(u) \mathrm{d} u,
\end{aligned}
$$


e, como $\left[\mathbb{E}_{h}\left(\beta_{0}, u\right)-\mathbb{E}_{h}\left(\bar{\beta}^{n}, u\right)\right]^{2}=o(1)$,

$$
\begin{aligned}
\left\langle\sum_{h=1}^{H-1} Y_{h}(t)\right\rangle= & \int_{0}^{t}\left[\sum_{k=1}^{K} \mathbb{I}_{k}(u) \frac{l_{k}}{\sum_{h=1}^{H-1} \sigma_{k h}^{2}}\right]^{2} \sum_{h=1}^{H-1} \mathbb{V}_{h}\left(\beta_{0}, u\right) S_{h}^{(0)}\left(\beta_{0}, u\right) \alpha_{0 h}(u) \mathrm{d} u \\
& -\int_{0}^{t}\left[\sum_{k=1}^{K} \mathbb{I}_{k}(u) \frac{l_{k}}{\sum_{h=1}^{H-1} \sigma_{k h}^{2}}\right]^{2} \sum_{h=1}^{H-1} v_{h}\left(\beta_{0}, u\right) s_{h}^{(0)}\left(\beta_{0}, u\right) \alpha_{0 h}(u) \mathrm{d} u \\
& +\int_{0}^{t}\left[\sum_{k=1}^{K} \mathbb{I}_{k}(u) \frac{l_{k}}{\sum_{h=1}^{H-1} \sigma_{k h}^{2}}\right]^{2} \sum_{h=1}^{H-1} v_{h}\left(\beta_{0}, u\right) s_{h}^{(0)}\left(\beta_{0}, u\right) \alpha_{0 h}(u) \mathrm{d} u \\
= & \int_{0}^{t}\left[\sum_{k=1}^{K} \mathbb{I}_{k}(u) \frac{l_{k}}{\sum_{h=1}^{H-1} \sigma_{k h}^{2}}\right]^{2} \\
& \cdot \sum_{h=1}^{H-1}\left[\mathbb{V}_{h}\left(\beta_{0}, u\right) S_{h}^{(0)}\left(\beta_{0}, u\right)-v_{h}\left(\beta_{0}, u\right) s_{h}^{(0)}\left(\beta_{0}, u\right)\right] \alpha_{0 h}(u) \mathrm{d} u \\
& +\int_{0}^{t}\left[\sum_{k=1}^{K} \mathbb{I}_{k}(u) \frac{l_{k}}{\sum_{h=1}^{H-1} \sigma_{k h}^{2}}\right]^{2} \sum_{h=1}^{H-1} v_{h}\left(\beta_{0}, u\right) s_{h}^{(0)}\left(\beta_{0}, u\right) \alpha_{0 h}(u) \mathrm{d} u \\
= & o_{p}(1)+\int_{0}^{t}\left[\sum_{k=1}^{K} \mathbb{I}_{k}(u) \frac{l_{k}}{\sum_{h=1}^{H-1} \sigma_{k h}^{2}} \sum_{h=1}^{H-1} v_{h}\left(\beta_{0}, u\right) s_{h}^{(0)}\left(\beta_{0}, u\right) \alpha_{0 h}(u) \mathrm{d} u .\right.
\end{aligned}
$$

Por continuidade de $\sum_{h=1}^{H-1} v_{h}\left(\beta_{0}, u\right) s_{h}^{(0)}\left(\beta_{0}, u\right) \alpha_{0 h}(u)$, temos

$$
\max _{1 \leq k \leq K} \sup _{u \in \mathbb{I}_{k}}\left|l_{k}^{-1}\left(\sum_{h=1}^{H-1} \sigma_{k h}^{2}\right)-\sum_{h=1}^{H-1} v_{h}\left(\beta_{0}, u\right) s_{h}^{(0)}\left(\beta_{0}, u\right) \alpha_{0 h}(u)\right| \rightarrow 0 .
$$


Assim,

$$
\begin{aligned}
\left\langle\sum_{h=1}^{H-1} Y_{h}(t)\right\rangle & =\int_{0}^{t}\left[\sum_{k=1}^{K} \mathbb{I}_{k}(u) \frac{l_{k}}{\sum_{h=1}^{H-1} \sigma_{k h}}\right]^{2} \sum_{h=1}^{H-1} v_{h}\left(\beta_{0}, u\right) s_{h}^{(0)}\left(\beta_{0}, u\right) \alpha_{0 h}(u) \mathrm{d} u \\
& =\int_{0}^{t}\left(\sum_{h=1}^{H-1} v_{h}\left(\beta_{0}, u\right) s_{h}^{(0)}\left(\beta_{0}, u\right) \alpha_{0 h}(u)\right)^{-1} \mathrm{~d} u
\end{aligned}
$$

Usando a Suposição A(1) e o Lema A.1, concluímos que

$$
\left\langle\sum_{h=1}^{H-1} Y_{h}(t)\right\rangle \stackrel{p}{\rightarrow} \int_{0}^{t}\left[\sum_{h=1}^{H-1} v_{h}\left(\beta_{0}, u\right) s_{h}^{(0)}\left(\beta_{0}, u\right) \alpha_{0 h}(u)\right]^{-1} \mathrm{~d} u
$$

que é uma função contínua, satisfazendo a primeira condição. Com relação à segunda condição, temos de considerar o processo associado ao primeiro fator (3.19) e mostrar que seu processo de variação previsível converge para zero em probabilidade, para finalmente concluir pelo Teorema Central do Limite de Rebolledo que o processo converge para um processo gaussiano.

De acordo com o resultado encontrado em Andersen et al. (1993), se para cada $n=1,2, \ldots$, $N_{h}(t)$ é um processo de contagem com processo de intensidade $\Lambda_{h}(t), H_{h}(t)$ é um processo previsível, em que

$$
H_{h}(u)=\frac{1}{\sqrt{n}} \sum_{j=1}^{n}\left[\sum_{k=1}^{K} \mathbb{I}_{k}(u) \frac{l_{k}}{\sum_{h=1}^{H-1} \sigma_{k h}^{2}}\right]\left[X_{j}(u)-\mathbb{E}_{h}\left(\bar{\beta}^{n}, u\right)\right]
$$

O processo de variação previsível de saltos é dado por

$$
Y_{h \epsilon}(t)=\sum_{i=1}^{n} \int_{0}^{t} H_{h}(u) \mathbb{I}\left\{H_{h}(u)>\epsilon\right\} \mathrm{d} M_{h i}(u), \quad h=1, \ldots, H-1 .
$$

O correspondente processo de variação previsível é

$$
\left\langle Y_{h \epsilon}(t)\right\rangle=\sum_{i=1}^{n} \int_{0}^{t}\left[H_{h}(u)\right]^{2} \mathbb{I}\left\{H_{h}(u)>\epsilon\right\} \mathrm{d} \Lambda_{h i}(u), \quad h=1, \ldots, H-1
$$


Assim,

$$
\begin{aligned}
\left\langle Y_{h \epsilon}(t)\right\rangle= & \int_{0}^{t} \frac{1}{n} \sum_{j=1}^{n}\left[X_{j}-\mathbb{E}_{h}\left(\bar{\beta}^{n}, u\right)\right]^{2} e^{\beta_{0}(u) X_{j}} Y_{j h}(u) \alpha_{0 h}(u)\left[\sum_{k=1}^{K} \mathbb{I}_{k}(u) \frac{l_{k}}{\sum_{h=1}^{H-1} \sigma_{k h}^{2}}\right]^{2} \\
& . \mathbb{I}\left\{u:\left|X_{j}(u)-\mathbb{E}_{h}\left(\bar{\beta}^{n}, u\right)\right|>\epsilon \sqrt{n}\left[\sum_{k=1}^{K} \mathbb{I}_{k}(u) \frac{l_{k}}{\sum_{h=1}^{H-1} \sigma_{k h}^{2}}\right]\right\} \mathrm{d} u
\end{aligned}
$$

é $o_{p}(1)$ para cada $\epsilon>0$.

Para mostrar isso, lembramos que $\min _{1 \leq k \leq K} \frac{\sum_{h=1}^{H-1} \sigma_{k h}^{2}}{l_{k}} \geq L \Rightarrow \frac{l_{k}}{\sum_{h=1}^{H-1} \sigma_{k h}^{2}} \leq L^{-1}=O(1)$. Então a condição acima está satisfeita se

$$
\begin{aligned}
& \int_{0}^{\tau} \frac{1}{n} \sum_{j=1}^{n}\left[X_{j}(u)-\mathbb{E}_{h}\left(\bar{\beta}^{n}, u\right)\right]^{2} e^{\beta_{0}(u) X_{j}(u)} Y_{j h}(u) \alpha_{0 h}(u) \\
& . \mathbb{I}\left\{u:\left|X_{j}(u)-\mathbb{E}_{h}\left(\bar{\beta}^{n}, u\right)\right|>\epsilon \sqrt{n}\right\} \mathrm{d} u=o_{p}(1), \quad \forall \epsilon>0 .
\end{aligned}
$$

Murphy \& Sen (1991) e Pedroso de Lima \& Sen (1999) mostram o resultado acima usando a desigualdade

$$
|a-b|^{2} \mathbb{I}\{|a-b|>\epsilon\} \leq 4|a|^{2} \mathbb{I}\{|a|>\epsilon / 2\}+4|b|^{2} \mathbb{I}\{|b|>\epsilon / 2\}
$$

Como

$$
\left\langle\sum_{h=1}^{H-1} Y_{h}(t)\right\rangle=\sum_{h=1}^{H-1}\left\langle Y_{h}(t)\right\rangle+\sum_{h \neq l}\left\langle Y_{h}(t), Y_{l}(t)\right\rangle
$$

mas $\sum_{h \neq l}\left\langle Y_{h}(t), Y_{l}(t)\right\rangle=0$, admitindo que um indivíduo não executa duas transições simultaneamente, $\left\langle\sum_{h=1}^{H-1} Y_{h \epsilon}(t)\right\rangle=\sum_{h=1}^{H-1}\left\langle Y_{h \epsilon}(t)\right\rangle=o_{p}(1)$.

Para completar, um estimador consistente da variância assintótica é provado no teorema 
apresentado na próxima subseção.

\subsubsection{Consistência do estimador da covariância}

Uma possível escolha para o estimador da covariância é dada pela inversa da segunda derivada da log-verossimilhança,

$$
\int_{0}^{t}\left[-\sum_{h=1}^{H-1} \sum_{k=1}^{K} \mathbb{I}_{k}(u)\left(l_{k} n\right)^{-1} \frac{\partial^{2}}{\partial \beta_{k}^{2}} \mathcal{L}_{n h}\left(\widehat{\beta}^{n}\right)\right]^{-1} \mathrm{~d} u
$$

A consistência desse estimador é considerada no teorema a seguir.

Teorema 3.3. Suponha

(a) $\lim _{n} n\|l\|^{4}=\infty, \quad \lim _{n}\|l\|^{2}=0$

(b) $A(1), A(3), C, D(1), D(3) \quad e$

(c) $\lim \sup _{n} \frac{l_{(K)}}{l_{(1)}}<\infty$;

então,

$$
\begin{aligned}
& \sup _{0 \leq t \leq \tau} \mid \int_{0}^{t}\left[-\sum_{h=1}^{H-1} \sum_{k=1}^{K} \mathbb{I}_{k}(u)\left(l_{k} n\right)^{-1} \frac{\partial^{2}}{\partial \beta_{k}^{2}} \mathcal{L}_{n h}\left(\widehat{\beta}^{n}\right)\right]^{-1} d u \\
& -\int_{0}^{t}\left[\sum_{h=1}^{H-1} v_{h}\left(\beta_{0}, u\right) s_{h}^{(0)}\left(\beta_{0}, u\right) \alpha_{0 h}(u)\right]^{-1} d u \mid=o_{p}(1),
\end{aligned}
$$

quando $n \rightarrow \infty$. 


\section{Demonstração}

$$
\begin{aligned}
\sup _{0 \leq t \leq \tau} \mid \int_{0}^{t}\left[-\sum_{h=1}^{H-1} \sum_{k=1}^{K} \mathbb{I}_{k}(u)\left(l_{k} n\right)^{-1} \frac{\partial^{2}}{\partial \beta_{k}^{2}} \mathcal{L}_{n h}\left(\widehat{\beta}^{n}\right)\right]^{-1} \mathrm{~d} u \\
-\int_{0}^{t}\left[\sum_{h=1}^{H-1} v_{h}\left(\beta_{0}, u\right) s_{h}^{(0)}\left(\beta_{0}, u\right) \alpha_{0 h}(u)\right]^{-1} \mathrm{~d} u \mid \\
\leq \int_{0}^{\tau}\left|-\sum_{h=1}^{H-1} \sum_{k=1}^{K} \mathbb{I}_{k}(u)\left(l_{k} n\right)^{-1} \frac{\partial^{2}}{\partial \beta_{k}^{2}} \mathcal{L}_{n h}\left(\widehat{\beta}^{n}\right)-\sum_{h=1}^{H-1} v_{h}\left(\beta_{0}, u\right) s_{h}^{(0)}\left(\beta_{0}, u\right) \alpha_{0 h}(u)\right| \mathrm{d} u \\
\sup _{0 \leq u \leq \tau}\left[\left(\sum_{h=1}^{H-1} v_{h}\left(\beta_{0}, u\right) s_{h}^{(0)}\left(\beta_{0}, u\right) \alpha_{0 h}(u)\right)\left(-\sum_{h=1}^{H-1} \sum_{k=1}^{K} \mathbb{I}_{k}(u)\left(l_{k} n\right)^{-1} \frac{\partial^{2}}{\partial \beta_{k}^{2}} \mathcal{L}_{n h}\left(\widehat{\beta}^{n}\right)\right)\right]^{(3.2}
\end{aligned}
$$

Vamos observar o primeiro fator (3.25).

$$
\begin{aligned}
& \int_{0}^{\tau}\left|-\sum_{h=1}^{H-1} \sum_{k=1}^{K} \mathbb{I}_{k}(u)\left(l_{k} n\right)^{-1} \frac{\partial^{2}}{\partial \beta_{k}^{2}} \mathcal{L}_{n h}\left(\widehat{\beta}^{n}\right)-\sum_{h=1}^{H-1} v_{h}\left(\beta_{0}, u\right) s_{h}^{(0)}\left(\beta_{0}, u\right) \alpha_{0 h}(u)\right| \mathrm{d} u \\
\leq & \int_{0}^{\tau}\left|\sum_{h=1}^{H-1} \sum_{k=1}^{K} \mathbb{I}_{k}(u)\left(l_{k} n\right)^{-1}\left[\frac{\partial^{2}}{\partial \beta_{k}^{2}} \mathcal{L}_{n h}\left(\widehat{\beta}^{n}\right)-\frac{\partial^{2}}{\partial \beta_{k}^{2}} \mathcal{L}_{n h}\left(\bar{\beta}^{n}\right)\right]\right| \mathrm{d} u \\
& +\int_{0}^{\tau}\left|\sum_{h=1}^{H-1} \sum_{k=1}^{K} \mathbb{I}_{k}(u)\left[\left(l_{k} n\right)^{-1} \frac{\partial^{2}}{\partial \beta_{k}^{2}} \mathcal{L}_{n h}\left(\bar{\beta}^{n}\right)-l_{k}^{-1}\left(\sum_{h=1}^{H-1} \sigma_{k h}^{2}\right)\right]\right| \mathrm{d} u \\
& +\int_{0}^{\tau}\left|\sum_{h=1}^{H-1} \sum_{k=1}^{K} \mathbb{I}_{k}(u) l_{k}^{-1}\left(\sigma_{k h}^{2}\right)-\sum_{h=1}^{H-1} v_{h}\left(\beta_{0}, u\right) s_{h}^{(0)}\left(\beta_{0}, u\right) \alpha_{0 h}(u)\right| \mathrm{d} u .
\end{aligned}
$$

O segundo termo $(3.28)$ da soma acima é $o_{p}(1)$ pelo Lema A.2, e o terceiro termo também é $o_{p}(1)$ por continuidade de $v_{h}\left(\beta_{0}, u\right) s_{h}^{(0)}\left(\beta_{0}, u\right) \alpha_{0 h}(u)$. Para o primeiro termo 3.27), temos 
que

$$
\begin{aligned}
& \int_{0}^{\tau}\left|\sum_{h=1}^{H-1} \sum_{k=1}^{K} \mathbb{I}_{k}(u)\left(l_{k} n\right)^{-1}\left[\frac{\partial^{2}}{\partial \beta_{k}^{2}} \mathcal{L}_{n h}\left(\widehat{\beta}^{n}\right)-\frac{\partial^{2}}{\partial \beta_{k}^{2}} \mathcal{L}_{n h}\left(\bar{\beta}^{n}\right)\right]\right| \mathrm{d} u \\
\leq & \int_{0}^{\tau} \sum_{h=1}^{H-1}\left|\mathbb{V}\left(\widehat{\beta}^{n}, u\right)-\mathbb{V}\left(\bar{\beta}^{n}, u\right)\right| \mathrm{d} \bar{N}_{h}(u) \quad\left(\bar{N}_{h}(u)=n^{-1} \sum_{i=1}^{n} N_{i s}(u)\right) \\
= & \sum_{h=1}^{H-1} \sum_{k=1}^{K}\left|\widehat{\beta}_{k}^{n}-\bar{\beta}_{k}\right| \int_{0}^{\tau}\left|\mathbb{I}_{k}(u) \frac{\partial}{\partial \beta_{k}} \mathbb{V}\left(\beta^{*}, u\right)\right| \mathrm{d} \bar{N}_{h}(u) \\
& \left(\text { em que }\left\|\beta^{*}-\bar{\beta}^{n}\right\| \leq\left\|\widehat{\beta}^{n}-\bar{\beta}^{n}\right\|\right) \\
= & o_{p}(1),
\end{aligned}
$$

pela Suposição A(3), Lema A.1 e o fato de que $\left\|\widehat{\beta}^{n}-\bar{\beta}^{n}\right\| \stackrel{p}{\rightarrow} 0$ quando $n \rightarrow \infty$.

O segundo fator (3.26) é $O_{p}(1)$ e pode ser provado da mesma forma anterior e pela Suposição $\mathrm{C}(2)$, isto é,

$$
\begin{aligned}
& \sup _{0 \leq u \leq \tau}\left[\left(\sum_{h=1}^{H-1} v_{h}\left(\beta_{0}, u\right) s_{h}^{(0)}\left(\beta_{0}, u\right) \alpha_{0 h}(u)\right)\left(\sum_{h=1}^{H-1} \sum_{k=1}^{K} \mathbb{I}_{k}(u)\left(l_{k} n\right)^{-1} \frac{\partial^{2}}{\partial \beta_{k}^{2}} \mathcal{L}_{n h}\left(\widehat{\beta}^{n}\right)\right)\right]^{-1} \\
= & \sup _{0 \leq u \leq \tau}\left[\left(\sum_{h=1}^{H-1} O(1)\right)\left(\sum_{h=1}^{H-1} \sum_{k=1}^{K} \mathbb{I}_{k}(u)\left(l_{k} n\right)^{-1} \frac{\partial^{2}}{\partial \beta_{k}^{2}} \mathcal{L}_{n h}\left(\widehat{\beta}^{n}\right)\right)\right]^{-1} \quad(\text { por C2 }) \\
= & O(1) \sup _{0 \leq u \leq \tau}\left[\sum_{h=1}^{H-1} \sum_{k=1}^{K} \mathbb{I}_{k}(u)\left(l_{k} n\right)^{-1} \frac{\partial^{2}}{\partial \beta_{k}^{2}} \mathcal{L}_{n h}\left(\widehat{\beta}^{n}\right)\right] \\
= & O(1) \sup _{0 \leq u \leq \tau}\left[\sum_{h=1}^{H-1} \sum_{k=1}^{K} \mathbb{I}_{k}(u)\left(l_{k} n\right)^{-1} \frac{\partial^{2}}{\partial \beta_{k}^{2}} \mathcal{L}_{n h}\left(\widehat{\beta}^{n}\right)\right. \\
& \left.+\sum_{h=1}^{H-1} \sum_{k=1}^{K} \mathbb{I}_{k}(u)\left(l_{k} n\right)^{-1} \frac{\partial^{2}}{\partial \beta_{k}^{2}} \mathcal{L}_{n h}\left(\bar{\beta}^{n}\right)-\sum_{h=1}^{H-1} \sum_{k=1}^{K} \mathbb{I}_{k}(u)\left(l_{k} n\right)^{-1} \frac{\partial^{2}}{\partial \beta_{k}^{2}} \mathcal{L}_{n h}\left(\bar{\beta}^{n}\right)\right]^{-1}
\end{aligned}
$$




$$
\begin{aligned}
= & O(1) \sup _{0 \leq u \leq \tau}\left[\sum_{h=1}^{H-1} \sum_{k=1}^{K} \mathbb{I}_{k}(u)\left(l_{k} n\right)^{-1}\left(\frac{\partial^{2}}{\partial \beta_{k}^{2}} \mathcal{L}_{n h}\left(\widehat{\beta}^{n}\right)-\frac{\partial^{2}}{\partial \beta_{k}^{2}} \mathcal{L}_{n h}\left(\bar{\beta}^{n}\right)\right)\right. \\
& \left.-\sum_{h=1}^{H-1} \sum_{k=1}^{K} \mathbb{I}_{k}(u)\left(l_{k} n\right)^{-1} \frac{\partial^{2}}{\partial \beta_{k}^{2}} \mathcal{L}_{n h}\left(\bar{\beta}^{n}\right)\right]^{-1} \\
\leq & O(1) \sup _{0 \leq u \leq \tau}\left[o_{p}(1) \sum_{h=1}^{H-1} \sum_{k=1}^{K} \mathbb{I}_{k}(u)\left(l_{k} n\right)^{-1} \frac{\partial^{2}}{\partial \beta_{k}^{2}} \mathcal{L}_{n h}\left(\bar{\beta}^{n}\right)\right]^{-1} \\
= & O_{p}(1)\left[\sum_{h=1}^{H-1} \sum_{k=1}^{K} \mathbb{I}_{k}(u)\left(\left(l_{k} n\right)^{-1} \frac{\partial^{2}}{\partial \beta_{k}^{2}} \mathcal{L}_{n h}\left(\bar{\beta}^{n}\right)+\frac{\sigma_{k h}^{2}}{l_{k}}-\frac{\sigma_{k h}^{2}}{l_{k}}\right)\right]^{-1} \text { (Resultado A.4) } \\
\leq & O_{p}(1)\left[\sum_{h=1}^{H-1} \frac{\sigma_{k h}^{2}}{l_{k}}\right]^{-1}=O_{p}(1)
\end{aligned}
$$




\section{Capítulo 4}

\section{Aplicação a dados reais}

Neste capítulo, apresentamos duas aplicações do modelo multi-estados aos dados de Restiffe (2007), que motivaram a nossa pesquisa. Na primeira aplicação, usamos o modelo homogêneo (2.10) e, na segunda, usamos o modelo não homogêneo 2.16), proposto em nosso trabalho.

O conjunto de dados é composto por 108 indivíduos, sendo 65 bebês RNPT e 43 RNT. Em cada avaliação mensal é atribuído um escore que varia de 1 a 58. Para a análise descrita neste capítulo, agrupamos os 58 escores de forma a obter cinco estados principais, conforme os movimentos apresentados pelos bebês que são descritos a seguir.

Estado 1 Rola da posição de barriga para baixo para barriga para cima, sustenta a cabeça e senta com apoio das mãos;

Estado 2 Senta sem apoio e rola de barriga para cima para barriga para baixo;

Estado 3 Fica de pé sozinho com apoio, engatinha e anda pelos móveis;

Estado 4 Anda sozinho, mas sem firmeza;

Estado 5 Pega o brinquedo no chão de cócoras e volta a ficar de pé. 
Assim, cada estágio de desenvolvimento neuropsicomotor das crianças representa um estado. As crianças transitam do estado atual ao seguinte até atingir o escore máximo (estado 5). Trata-se, portanto, de uma situação em que o modelo progressivo é o mais indicado.

Nesse conjunto de dados $10 \%$ dos bebês deixam o estudo antes do término sem ter atingido o estado 5. Além disso, estamos supondo que o tempo observado é o tempo exato da transição.

A matriz de intensidades para as possíveis transições é dada por

$$
\boldsymbol{\Lambda}(t)=\left[\begin{array}{ccccc}
-\lambda_{12}(t) & \lambda_{12}(t) & 0 & 0 & 0 \\
0 & -\lambda_{23}(t) & \lambda_{23}(t) & 0 & 0 \\
0 & 0 & -\lambda_{34}(t) & \lambda_{34}(t) & 0 \\
0 & 0 & 0 & -\lambda_{45}(t) & \lambda_{45}(t) \\
0 & 0 & 0 & 0 & 0
\end{array}\right]
$$

Cada componente não-nulo da matriz (4.1) é descrito por uma função que pode ser dependente do tempo (não homogêneo) ou não (homogêneo). No modelo homogêneo, consideramos a intensidade de transição da forma $\lambda_{h}=\alpha_{0 h} e^{\beta_{h}^{\prime} X}$, em que $\beta_{h}$ é o coeficiente de regressão para a covariável grupo $\left(\mathrm{X}=0\right.$ : $\mathrm{RNT}$ e $\mathrm{X}=1$ : RNPT) e $\alpha_{0 h}$ é a intensidade de transição do grupo de bebês RNT, para a transição $h \rightarrow h+1$. Note que os coeficientes $\beta_{h}$ são constantes no tempo mas dependem do tipo de transição executada. Assim, a matriz de transição $\Lambda(t)$ é constante no tempo.

No modelo não homogêneo consideramos a intensidade de transição em que tanto a função de risco basal como o coeficiente para a covariável grupo são dependentes do tempo, isto é, $\lambda_{h}(t, X)=\alpha_{0 h}(t) e^{\beta^{\prime}(t) X} Y_{h}(t) \operatorname{com} h=1, \ldots, H-1$, o que resulta em uma matriz de intensidade de transição dependente do tempo.

\subsection{Modelo markoviano homogêneo}

Usando a biblioteca msm desenvolvida por Jackson (2007) no software R, que utiliza o método da máxima verossimilhança, obtemos as estimativas de $\lambda_{h}$ apresentadas na Tabela 
4.1. com a idade corrigida. Não há indicação de diferenças nas intensidades de transição entre

Tabela 4.1: Estimativas de $\lambda_{h j}$ e respectivos intervalos de confiança (IC), por grupo.

\begin{tabular}{ccccc}
\hline Grupo & Transiç̧̃̃o & Parâmetro & Estimativa & IC $(95 \%)$ \\
\hline \multirow{3}{*}{ RNT } & $1 \rightarrow 2$ & $\lambda_{12}$ & 0,25 & {$[0,19 ; 0,34]$} \\
& $2 \rightarrow 3$ & $\lambda_{23}$ & 0,34 & {$[0,25 ; 0,46]$} \\
& $3 \rightarrow 4$ & $\lambda_{34}$ & 0,35 & {$[0,26 ; 0,48]$} \\
& $4 \rightarrow 5$ & $\lambda_{45}$ & 0,45 & {$[0,33 ; 0,61]$} \\
\hline \multirow{3}{*}{ RNPT } & $1 \rightarrow 2$ & $\lambda_{12}$ & 0,21 & {$[0,17 ; 0,27]$} \\
& $2 \rightarrow 3$ & $\lambda_{23}$ & 0,32 & {$[0,25 ; 0,41]$} \\
& $3 \rightarrow 4$ & $\lambda_{34}$ & 0,30 & {$[0,23 ; 0,38]$} \\
& $4 \rightarrow 5$ & $\lambda_{45}$ & 0,36 & {$[0,28 ; 0,47]$} \\
\hline
\end{tabular}

os grupos.

Estimativas de $\beta_{h}$ são apresentadas na Tabela 4.2, com a idade corrigida e não indicam que a covariável grupo seja significante para as transições, pois o zero pertence a todos os intervalos de confiança.

Tabela 4.2: Estimativas de $\beta_{h j}$ e respectivos intervalos de confiança (IC).

\begin{tabular}{cccc}
\hline Transição & Parâmetro & Estimativa & IC $(95 \%)$ \\
\hline $1 \rightarrow 2$ & $\beta_{12}$ & $-0,15$ & {$[-0,54 ; 0,23]$} \\
$2 \rightarrow 3$ & $\beta_{23}$ & $-0,06$ & {$[-0,45 ; 0,33]$} \\
$3 \rightarrow 4$ & $\beta_{34}$ & $-0,18$ & {$[-0,57 ; 0,21]$} \\
$4 \rightarrow 5$ & $\beta_{45}$ & $-0,22$ & {$[-0,62 ; 0,18]$} \\
\hline
\end{tabular}

Ao observarmos o tempo médio de permanência, obtido por meio de (2.3), na Tabela 4.3. notamos que os RNPT demoram mais tempo em cada estado, indicando que eles se desenvolvem mais lentamente que os RNT. Entretanto, os intervalos de confiança dos tempos de permanência em cada estado estão sobrepostos, o que nos faz concluir que não há diferença significativa entre os tempos de permanência dos dois grupos, qualquer que seja o estado.

As estimativas da razão das intensidades de transição estão na Tabela 4.4. Elas indicam que o desenvolvimento de bebês recém-nascidos é semelhante nos dois grupos, pois todos os intervalos de confiança das razões das intensidades de transição contêm o valor 1. 
Tabela 4.3: Estimativa do tempo médio (meses) de permanência em cada estado, para os grupos de RNT e RNPT.

\begin{tabular}{ccccc}
\hline Grupo & Estados & Tempo & e.p. & IC $(95 \%)$ \\
\hline \multirow{3}{*}{ RNT } & 1 & 4,00 & 0,60 & {$[2,95 ; 5,34]$} \\
& 2 & 2,93 & 0,45 & {$[2,16 ; 3,96]$} \\
& 3 & 2,80 & 0,42 & {$[2,08 ; 3,77]$} \\
& 4 & 2,22 & 0,34 & {$[1,63 ; 3,00]$} \\
\hline \multirow{3}{*}{ RNPT } & 1 & 4,64 & 0,57 & {$[3,64 ; 5,91]$} \\
& 2 & 3,11 & 0,39 & {$[2,42 ; 3,98]$} \\
& 3 & 3,36 & 0,42 & {$[2,63 ; 4,30]$} \\
& 4 & 2,75 & 0,37 & {$[2,13 ; 3,57]$} \\
\hline
\end{tabular}

Tabela 4.4: Estimativas da razão das intensidades de transição.

\begin{tabular}{ccc}
\hline Transição & Razão de Taxas & IC $(95 \%)$ \\
\hline $1 \rightarrow 2$ & 0,85 & {$[0,58 ; 1,26]$} \\
$2 \rightarrow 3$ & 0,94 & {$[0,63 ; 1,39]$} \\
$3 \rightarrow 4$ & 0,83 & {$[0,56 ; 1,22]$} \\
$4 \rightarrow 5$ & 0,80 & {$[0,54 ; 1,20]$} \\
\hline
\end{tabular}

Uma vez estimadas as intensidades de transição, podemos obter as probabilidades de transição (não apresentado aqui) de um estado a outro em um intervalo de tempo $t$ por meio do resultado obtido em (2.9). Assim, é possível prever o número de indivíduos em cada estado nos tempos pré-fixados. A Tabela 4.5 apresenta valores observados e esperados de cinco em cinco meses.

Discrepâncias entre os valores observados e os esperados são encontradas. Por exemplo, sob o modelo homogêneo, pela Tabela 4.5, há aproximadamente 40 crianças de dez meses que já estão no estado 5, entretanto nenhuma criança foi observada nesse estado. Outros casos observados nessa tabela indicam que o modelo não está bem ajustado.

Quando usamos o modelo homogêneo estamos considerando que a matriz de intensidade de transição $\boldsymbol{\Lambda}(t)$ é constante no tempo. Para o caso particular de bebês RNT e RNPT, equivale a afirmar que a intensidade de transição $h \rightarrow h+1$ é constante, qualquer que seja a idade, o que não parece adequado. Sendo assim, vamos considerar, a seguir, que cada componente da 
Tabela 4.5: Valor observado versus esperado, com idade corrigida.

\begin{tabular}{rrrrrrrr}
\hline & Mês & \multicolumn{4}{c}{ Estados } & Total \\
\cline { 2 - 7 } & & 1 & 2 & 3 & 4 & 5 & \\
\hline \multirow{4}{*}{ Observado } & 0 & 13 & 0 & 0 & 0 & 0 & 13 \\
& 5 & 102 & 6 & 0 & 0 & 0 & 108 \\
& 10 & 0 & 31 & 69 & 7 & 0 & 107 \\
& 15 & 0 & 0 & 6 & 22 & 72 & 100 \\
Esperado & 20 & 0 & 0 & 0 & 1 & 98 & 99 \\
\hline \multirow{5}{*}{} & 0 & 13,00 & 0 & 0 & 0 & 0 & 13 \\
& 5 & 34,25 & 30,96 & 23,74 & 11,17 & 7,86 & 108 \\
& 10 & 10,77 & 15,63 & 22,17 & 18,47 & 39,96 & 107 \\
& 15 & 3,19 & 5,70 & 11,13 & 12,37 & 67,56 & 100 \\
& 20 & 1,00 & 2,00 & 4,75 & 6,29 & 84,96 & 99 \\
\hline
\end{tabular}

matriz $\Lambda(t)$ é uma função que varia no tempo.

\subsection{Modelo markoviano não homogêneo}

Sob o modelo (2.16), estimativas de $\beta(t)$ são obtidas pelo método de histograma crivo descrito no Capítulo3, em que particionamos o tempo do estudo em intervalos e maximizamos a função de verossimilhança para esse espaço paramétrico. A função optim do software R foi utilizada para obter as estimativas.

A princípio não existe uma regra sobre quantos intervalos devem ser considerados, mas para se ter uma idéia do comportamento do estimador, vamos considerar cinco, dez e 20 intervalos. Para obter estimativas dos $\beta_{k}^{\prime} s$ em cada partição considerada, dividimos o tempo de forma a ter quantidades iguais de observações em cada intervalo considerado. Shoenfeld (1980) usa essa técnica de segmentação para estimar a função de risco $\lambda(t)$ e avaliar o ajuste em modelos de riscos proporcionais de Cox em análise de sobrevivência. Essa sugestão é seguida por Murphy (1993) para estimar o coeficiente dependente do tempo em uma aplicação com dados de sobrevivência.

Um aspecto importante no processo de estimação é garantir que em cada intervalo haja 
indivíduos de ambos os grupos; em caso contrário não é possível estimar a matriz de covariâncias.

Os intervalos de tempo, as estimativas de $\beta_{k}^{\prime} s$ e os respectivos erros padrão para partição com cinco intervalos são dados na Tabela 4.6 a seguir.

Tabela 4.6: Estimativas de $\beta_{k}$ e respectivos erros padrão com cinco intervalos do tempo (meses).

\begin{tabular}{cccccc}
\hline$k$ & 1 & 2 & 3 & 4 & 5 \\
\hline$I_{k}$ & {$[0,00 ; 6,80)$} & {$[6,80 ; 9,23)$} & {$[9,23 ; 11,60)$} & {$[11,60 ; 13,67)$} & {$[13,67 ; 22,17]$} \\
$\beta_{k}$ & 0,103 & $-0,252$ & $-0,397$ & $-0,581$ & $-0,707$ \\
e.p. & 0,222 & 0,221 & 0,219 & 0,225 & 0,274 \\
\hline
\end{tabular}

Notamos que a estimativa de $\beta(t)$ é maior que zero para o primeiro intervalo de tempo, enquanto para os demais intervalos a sua estimativa é negativa. Considerando intervalos de confiança de $\beta_{k}^{\prime} s$, com $95 \%$ de confiança, temos que o valor zero pertence aos três primeiros intervalos, sugerindo que os dois grupos de bebês se desenvolvem de forma semelhante. Isso é explicado pelo fato de que, nos primeiros meses de vida, são observados e pontuados os movimentos mais rudimentares que um recém-nascido é capaz de executar. As estimativas de $\beta_{4}$ e $\beta_{5}$ são menores que zero e seus intervalos de confiança com $95 \%$ de confiança de não incluem o zero, indicando que o desenvolvimento de RNPT vai se diferenciando cada vez mais em relação ao desenvolvimento de RNT. Os movimentos dessa fase são mais complexos e requerem nível maior de amadurecimento e, pelos resultados obtidos, os RNPT demoram mais tempo para atingir esses níveis. Sendo assim, para observar o catch-up do desenvolvimento neuropsicomotor entre os RNPT e RNT, seria necessário ampliar a escala AIMS, acrescentando mais posturas após a última definida na escala, tais como andar para trás, saltar, andar em linha reta etc. (Restiffe, 2007).

Assim, pode-se concluir que, ao contrário do que se esperava, o desenvolvimento neuropsicomotor dos RNPT e RNT, com a idade corrigida, é semelhante no início e se torna diferente com o aumento da complexidade dos movimentos e, portanto, deve-se corrigir completamente as idades dos RNPT até o fim da escala AIMS. Ou seja, corrigir a idade até os 18 meses não superestima o desenvolvimento neuropsicomotor dos bebês RNPT. 
A situação aqui considerada é progressiva. Os indivíduos transitam sempre para o estado seguinte, e tempos posteriores correspondem a transições posteriores. Comparando os valores de $\beta$ obtidos na Tabela 4.2 com os da Tabela 4.6, notamos que, sob o modelo homogêneo, apesar de afirmarmos que não há efeito de grupo, os valores de $\beta_{h j}$ correspondentes às últimas transições indicam o contrário. As últimas transições ocorrem nos últimos intervalos de tempo, e os valores estimados de $\beta_{k}^{\prime} s$ do modelo não homogêneo indicam que há, de fato, presença de efeito de grupo.

Estimativas com dez e 20 intervalos são apresentadas nas Tabelas 4.7 e 4.8 a seguir.

Tabela 4.7: Estimativas de $\beta_{k}$ e respectivos erros padrão com dez intervalos do tempo (meses).

\begin{tabular}{cccccc}
\hline$k$ & 1 & 2 & 3 & 4 & 5 \\
\hline$I_{k}$ & {$[0,00 ; 5,77)$} & {$[5,77 ; 6,80)$} & {$[6,80 ; 8,03)$} & {$[8,03 ; 9,23)$} & {$[9,23 ; 10,77)$} \\
$\beta_{k}$ & 0,072 & 0,134 & $-0,203$ & $-0,300$ & $-0,276$ \\
e.p. & 0,318 & 0,310 & 0,316 & 0,309 & 0,313 \\
\hline \hline$k$ & 6 & 7 & 8 & 9 & 10 \\
\hline$I_{k}$ & {$[10,77 ; 11,60)$} & {$[11,60 ; 12,67)$} & {$[12,67 ; 13,67)$} & {$[13,67 ; 14,53)$} & {$[14,53 ; 22,17]$} \\
$\beta_{k}$ & $-0,514$ & $-0,399$ & $-0,750$ & $-0,504$ & $-1,011$ \\
e.p. & 0,308 & 0,326 & 0,312 & 0,354 & 0,425 \\
\hline
\end{tabular}

Aumento do númeo de intervalos faz com que o número de observações por intervalo seja menor o que resulta no aumento da variabilidade das estimativas de $\beta_{k}^{\prime} s$ resultando maior instabilidade de suas estimativas.

No capítulo anterior vimos que, teoricamente, número maior de intervalos combinado com o aumento do tamanho amostral resultam na melhor aproximação da função $\beta(t)$ a ser estimada. Quando usamos dez intervalos com a mesma amostra usada com cinco intervalos, ainda percebe-se uma tendência da função $\beta(t)$, podendo manter as mesmas conclusões feitas com cinco intervalos. Entretanto, na situação com 20 intervalos não há respostas conclusivas em relação ao efeito de grupo ao longo do tempo (Figuras 4.1, 4.2 e 4.3). Assim, para considerarmos uma partição com maior número de intervalos é necessário maior tamanho amostral.

Os gráficos das Figuras 4.1, 4.2 e 4.3 mostram a função $\beta(t)$ estimada pelo método do histograma crivo com cinco, dez e 20 intervalos. 
Tabela 4.8: Estimativas de $\beta_{k}$ e respectivos erros padrão com 20 intervalos do tempo (meses).

\begin{tabular}{cccccc}
\hline$k$ & 1 & 2 & 3 & 4 & 5 \\
\hline$I_{k}$ & {$[0,00 ; 5,40)$} & {$[5,40 ; 5,73)$} & {$[5,73 ; 6,07)$} & {$[6,07 ; 6,67)$} & {$[6,67 ; 7,30)$} \\
$\beta_{k}$ & $-0,126$ & 0,204 & 0,248 & $-0,185$ & $-0,285$ \\
e.p. & 0,441 & 0,469 & 0,422 & 0,474 & 0,437 \\
\hline \hline$k$ & 6 & 7 & 8 & 9 & 10 \\
\hline$I_{k}$ & {$[7,30 ; 7,93)$} & {$[7,93 ; 8,67)$} & {$[8,67 ; 9,13]$} & {$[9,13 ; 9,70)$} & {$[9,70 ; 10,50)$} \\
$\beta_{k}$ & 0,254 & 0,327 & $-0,860$ & 0,518 & $-1,105$ \\
e.p. & 0,459 & 0,501 & 0,449 & 0,517 & 0,445 \\
\hline \hline$k$ & 11 & 12 & 13 & 14 & 15 \\
\hline$I_{k}$ & {$[10,50 ; 11,10)$} & {$[11,10 ; 11,43)$} & {$[11,43 ; 12,07]$} & {$[12,07 ; 12,57)$} & {$[12,57 ; 13,03)$} \\
$\beta_{k}$ & 0,014 & $-0,692$ & $-0,601$ & $-0,381$ & 0,033 \\
e.p. & 0,463 & 0,461 & 0,467 & 0,450 & 0,472 \\
\hline \hline$k$ & 16 & 17 & 18 & 19 & 20 \\
\hline$I_{k}$ & {$[13,03 ; 13,43)$} & {$[13,43 ; 14,00)$} & {$[14,00 ; 14,35)$} & {$[14,35 ; 15,13)$} & {$[15,13 ; 22,17]$} \\
$\beta_{k}$ & $-0,982$ & $-0,370$ & $-1,159$ & $-0,534$ & $-1,576$ \\
e.p. & 0,467 & 0,462 & 0,508 & 0,528 & 0,509 \\
\hline
\end{tabular}

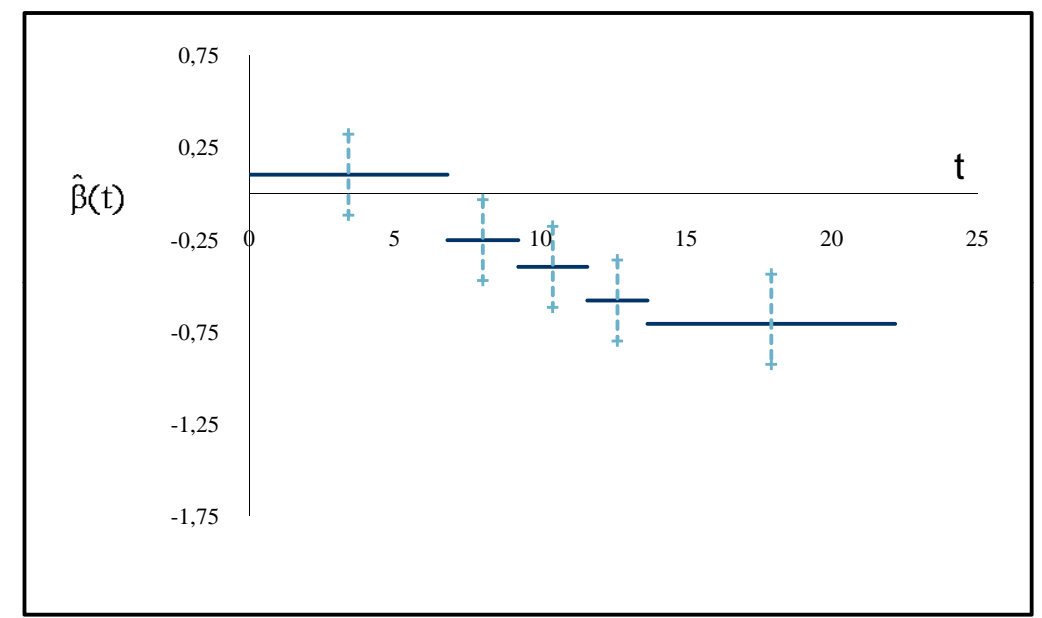

Figura 4.1: Estimativas de $\beta_{k}^{\prime} s$ com cinco intervalos e respectivos erros padrão. 


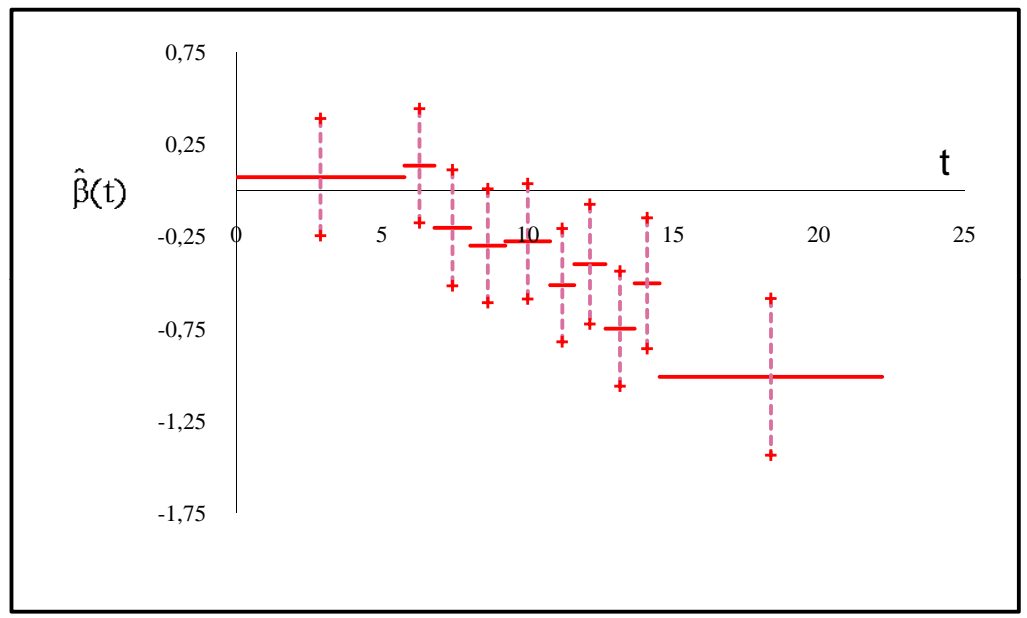

Figura 4.2: Estimativas de $\beta_{k}^{\prime} s$ com dez intervalos e respectivos erros padrão.

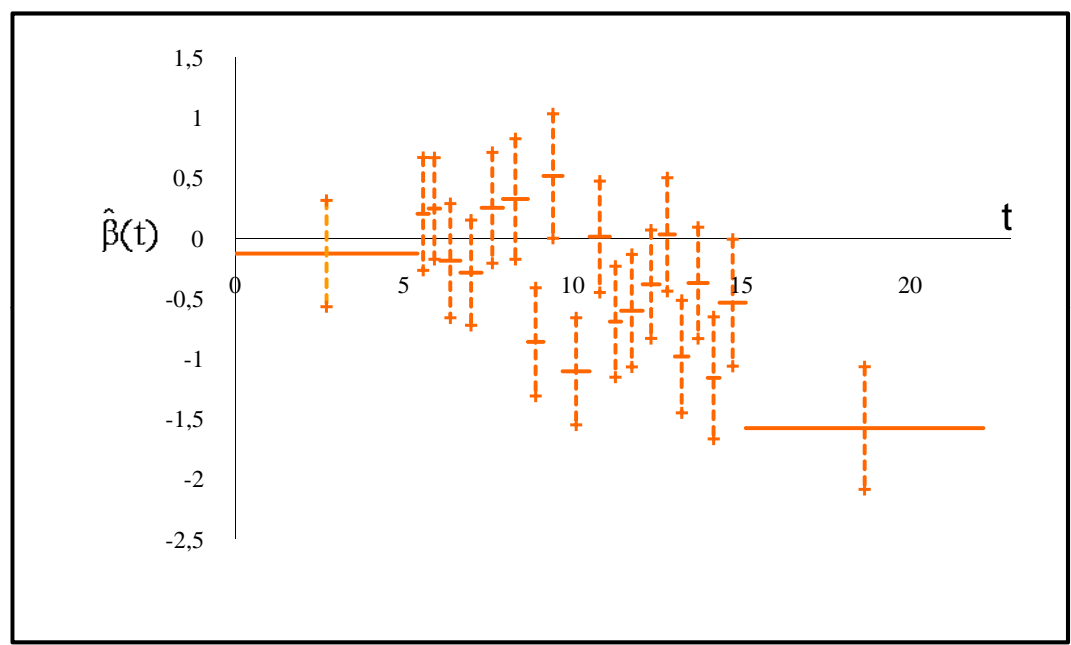

Figura 4.3: Estimativas de $\beta_{k}^{\prime} s$ com 20 intervalos e respectivos erros padrão. 
Notamos na Figura 4.3 que o critério de particionamento de tempo, com igual número de transições em cada intervalo, resultou em comprimentos maiores para o primeiro e último intervalos e menores para os intervalos intermediários.

As Figuras 4.1 e 4.2 mostram uma tendência decrescente de $\beta(t)$, indicando que não é possível observar a ocorrência de catch-up mesmo que os bebês sejam acompanhados até atingir o último estado englobado pela escala AIMS. Essa tendência é mais evidente quando consideramos apenas cinco intervalos do tempo e, quando aumentamos o número de intervalos, notamos a instabilidade dos valores dos parâmetros estimados (Figuras 4.2 e 4.3), pois o número de observações em cada intervalo fica reduzido.

Segundo Restiffe (2007), o catch-up ocorre em diferentes tempos para diferentes escalas, pois cada escala avalia diferentes aspectos do desenvolvimento de uma criança. No caso da escala AIMS, utilizada pela pesquisadora, ela avalia especificamente o desenvolvimento neuropsicomotor e é apropriada para bebês até 18 meses de idade.

Para reforçar as nossas conclusões, consideramos uma análise de sobrevivência com quatro eventos separadamente, como descrito a seguir:

Evento 1: tempo até atingir o estado 2;

Evento 2: tempo até atingir o estado 3;

Evento 3: tempo até atingir o estado 4;

Evento 4: tempo até atingir o estado 5.

Nos gráficos Kaplan-Meier das Figuras 4.4 a 4.7, notamos que as diferenças entre os dois grupos se mostram mais evidentes quando comparamos os dois últimos eventos. Essa é uma comparação um pouco rudimentar, mas serve para ilustrar que há diferença no desenvolvimento entre os dois grupos de bebês, considerando o Evento 3 ou 4 como sendo o objeto de estudo. E chegamos à mesma conclusão de que o grupo de bebês RNPT demora mais para atingir cada etapa do desenvolvimento.

Os modelos homogêneos são mais simples de aplicar que os não homogêneos e já existe programa disponível com o qual obtemos as estimativas dos parâmetros de interesse, o que não 


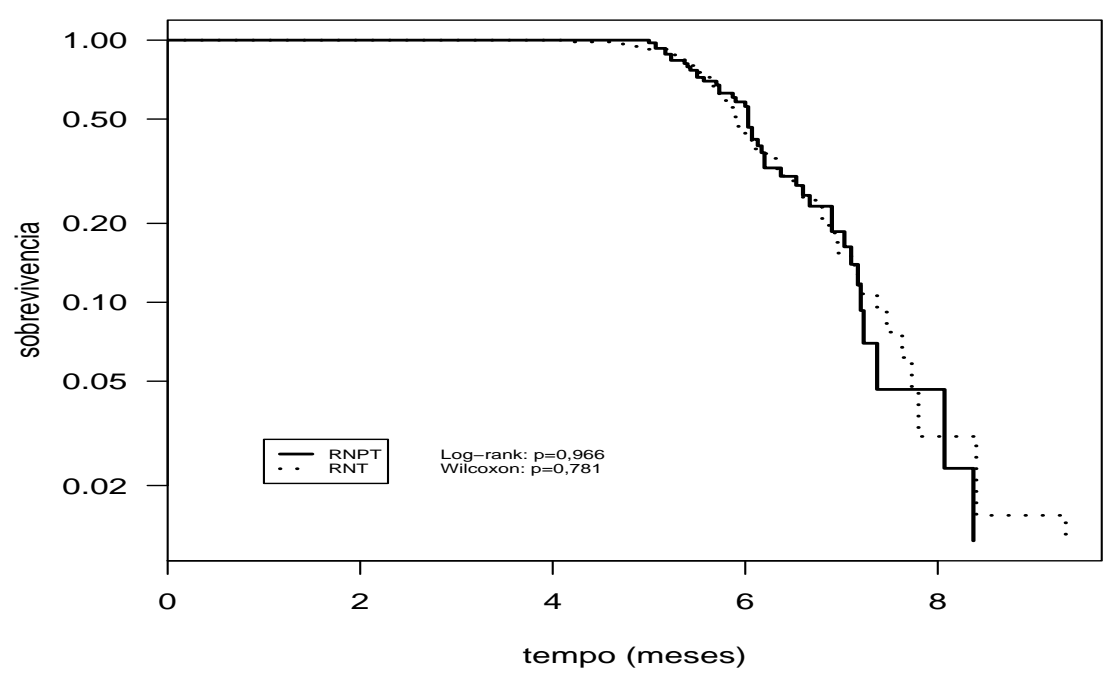

Figura 4.4: Curvas Kaplan-Meier para o Evento 1.

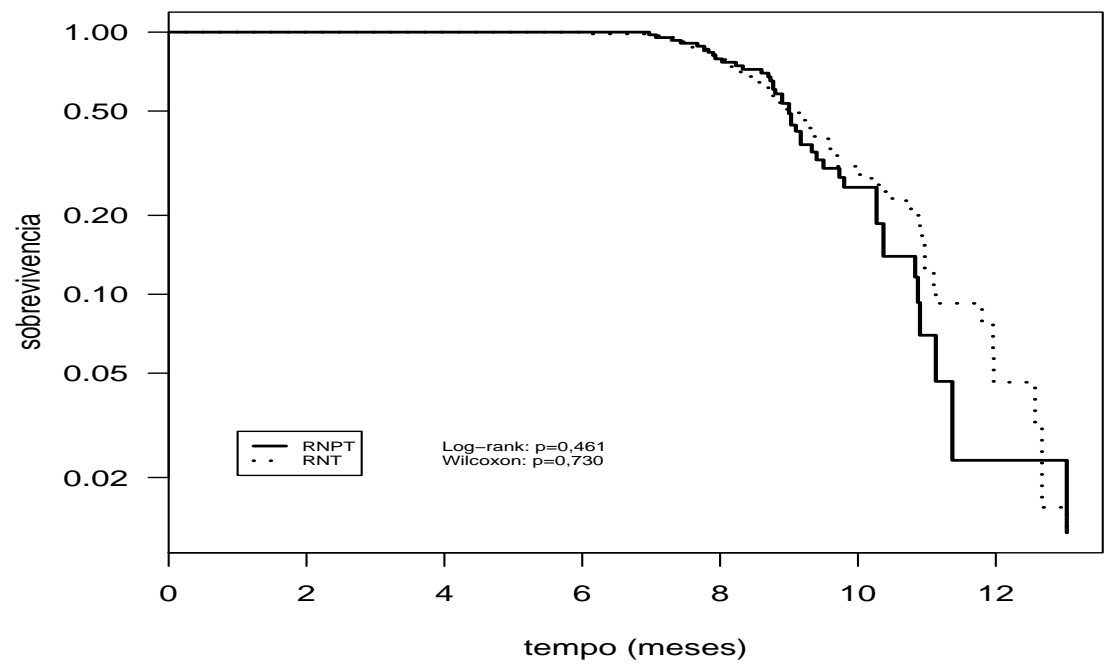

Figura 4.5: Curvas Kaplan-Meier para o Evento 2.

ocorre com o não homogêneo. Nesse caso, necessitamos escrever um programa específico com o objetivo de estimar os parâmetros de interesse. O programa utilizado para o caso particular 


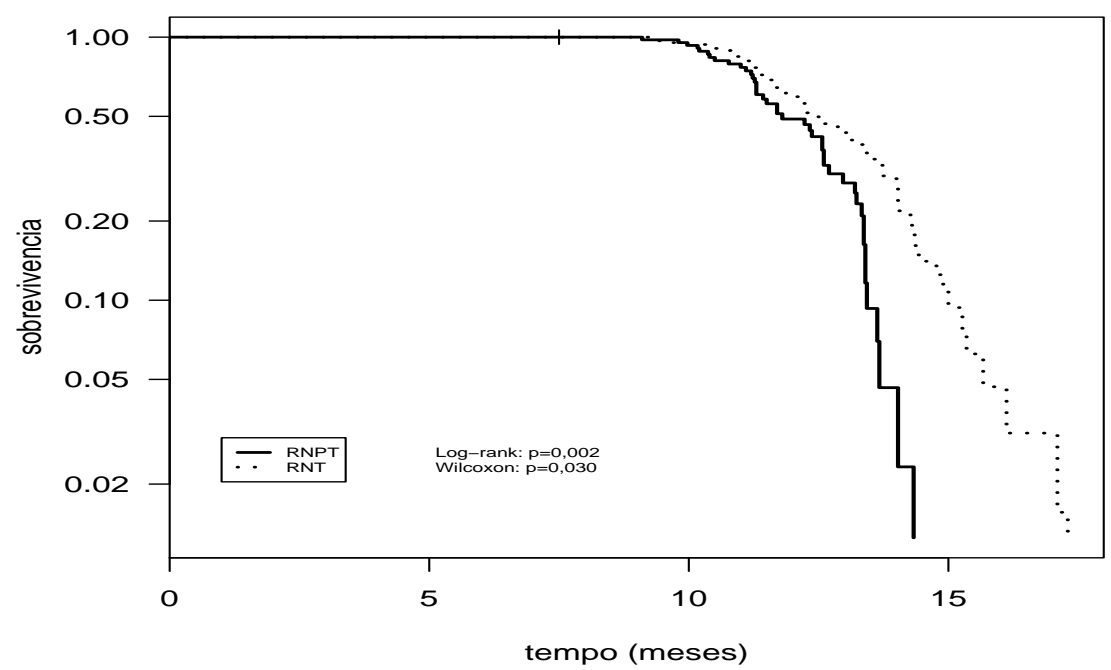

Figura 4.6: Curvas Kaplan-Meier para o Evento 3.

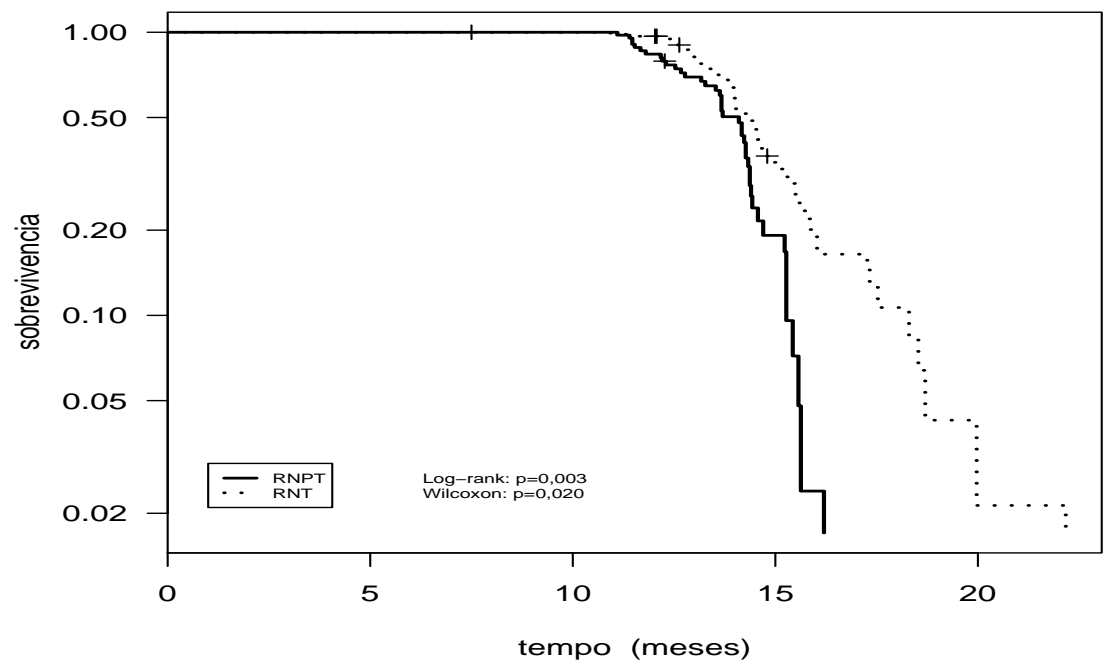

Figura 4.7: Curvas Kaplan-Meier para o Evento 4.

apresentado neste trabalho está disponível em www.ime.usp.br/ acarlos/multistate_sieves. 
No caso do modelo não homogêneo com coeficiente dependente do tempo, o aumento do número de intervalos resultou no aumento dos erros padrão e na instabilidade dos valores estimados dos parâmetros. Assim, consideramos réplicas da amostra inicial para estimar os parâmetros sob o modelo não homogêneo, e os resultados são apresentados na próxima seção.

\subsection{Réplicas do conjunto de dados original}

$\mathrm{Na}$ aplicação feita na seção anterior, vimos que o aumento do número de intervalos tornam instáveis os valores das estimativas de $\beta_{k}^{\prime} s$. Assim, um estudo de simulação com tamanho amostral maior se faz necessário, a fim de avaliar o processo de estimação de $\beta_{k}^{\prime} s$, quando aumentamos o número de intervalos.

Devido à dificuldade de simular dados com coeficientes dependentes do tempo para caso multi-estados causada pela estrutura de dependência entre os tempos de transição, consideramos réplicas da amostra inicial. Para tanto, a amostra original foi replicada e uma pequena perturbação foi acrescida para evitar empates dos tempos de transição. Aos tempos de transição de cada indivíduo foi adicionado um valor aleatório gerado de uma distribuição uniforme $U[-0,001 ; 0,001]$, e esse processo é repetido a cada réplica do conjunto de dados. Consideramos, ainda, casos em que são acrescidas censuras aleatórias.

O aumento do número de intervalos implica aumento do número de parâmetros e, portanto, aumento do tempo de estimação. Assim, para estimar $\beta_{k}^{\prime} s$, foi usado o histograma crivo com apenas cinco e dez intervalos e, para obter a partição do tempo, foi considerado o mesmo critério de número igual de observações em cada intervalo.

Aqui estamos considerando cinco réplicas do conjunto original de dados, conforme descrito na seção anterior, e lembramos que há aproximadamente $10 \%$ de censuras. As estimativas de $\beta_{k}^{\prime} s$ e seus erros padrão com cinco e dez intervalos são apresentados nas Tabelas 4.9 e 4.10 a seguir.

Os gráficos das Figuras 4.8 e 4.9 mostram a função $\beta(t)$ estimada através do método do histograma crivo. As estimativas de $\beta_{k}$ obtidas com cinco e dez intervalos ficaram mais 
Tabela 4.9: Estimativas de $\beta_{k}$ e respectivos erros padrão, com cinco intervalos.

\begin{tabular}{cccccc}
\hline$k$ & 1 & 2 & 3 & 4 & 5 \\
\hline$I_{k}$ & {$[0,00 ; 6,41)$} & {$[6,41 ; 9,09)$} & {$[9,09 ; 11,51)$} & {$[11,51 ; 13,67)$} & {$[13,67 ; 22,24]$} \\
$\beta_{k}$ & 0,076 & $-0,147$ & $-0,433$ & $-0,505$ & $-0,840$ \\
e.p. & 0,105 & 0,097 & 0,096 & 0,100 & 0,119 \\
\hline
\end{tabular}

Tabela 4.10: Estimativas de $\beta_{k}$ e respectivos erros padrão, com dez intervalos.

\begin{tabular}{cccccc}
\hline$k$ & 1 & 2 & 3 & 4 & 5 \\
\hline$I_{k}$ & {$[0,00 ; 5,59)$} & {$[5,59 ; 6,41)$} & {$[6,41 ; 7,81)$} & {$[7,81 ; 9,09)$} & {$[9,09 ; 10,56)$} \\
$\beta_{k}$ & $-0,021$ & 0,143 & $-0,047$ & $-0,244$ & $-0,436$ \\
e.p. & 0,163 & 0,138 & 0,139 & 0,135 & 0,136 \\
\hline \hline$k$ & 6 & 7 & 8 & 9 & 10 \\
\hline$I_{k}$ & {$[10,56 ; 11,51)$} & {$[11,51 ; 12,64)$} & {$[12,64 ; 13,67)$} & {$[13,67 ; 14,58)$} & {$[14,58 ; 22,24]$} \\
$\beta_{k}$ & $-0,431$ & $-0,376$ & $-0,629$ & $-0,733$ & $-1,025$ \\
e.p. & 0,137 & 0,144 & 0,139 & 0,149 & 0,194 \\
\hline
\end{tabular}

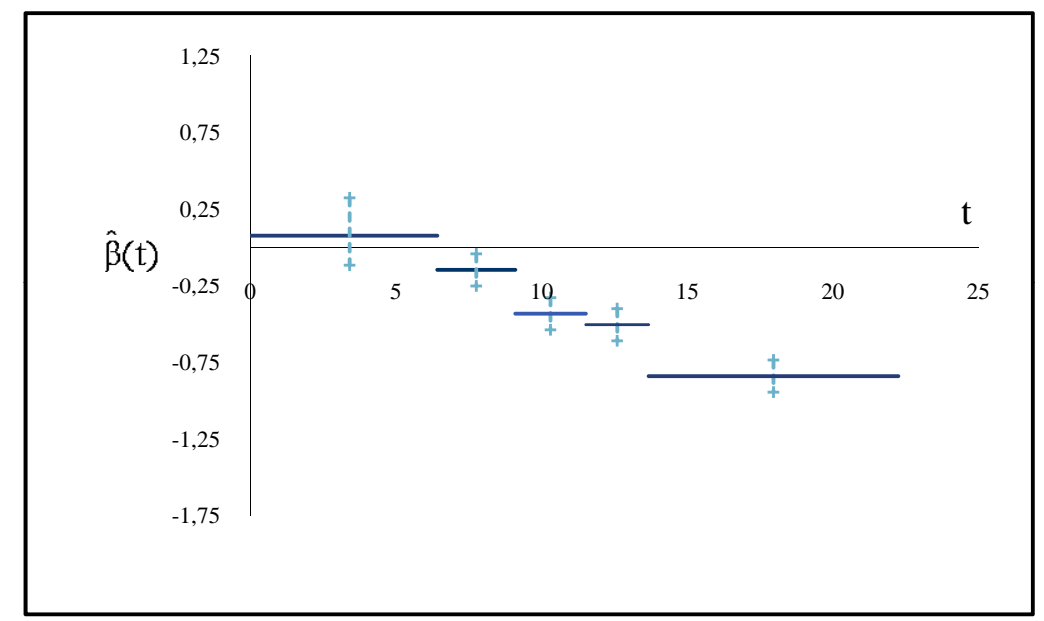

Figura 4.8: Estimativas de $\beta_{k}^{\prime} s$ com cinco intervalos e respectivos erros padrão.

estáveis com o aumento do tamanho amostral. Comparando a Tabela 4.6 com a Tabela 4.9 e a Tabelas 4.7 com a Tabela 4.10, notamos que aumentamos cinco vezes o tamanho amostral e respectivos erros padrão foram reduzidos praticamente à metade. Assim como ocorre com 


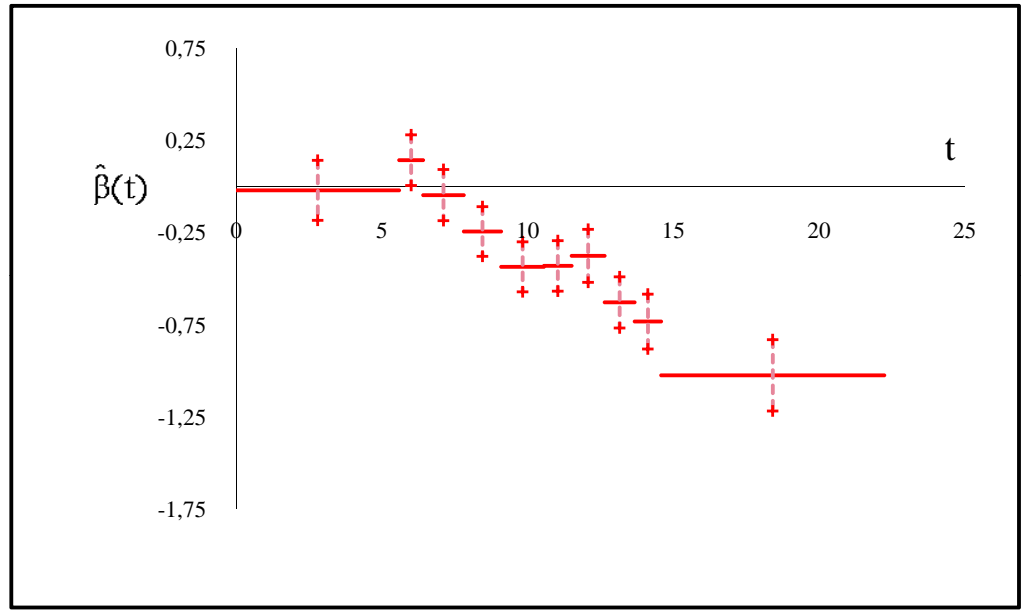

Figura 4.9: Estimativas de $\beta_{k}^{\prime} s$ com dez intervalos e respectivos erros padrão.

a amostra original, o aumento do número de intervalos gera aumento dos erros padrão das estimativas de $\beta_{k}^{\prime} s$, pois em cada intervalo temos número reduzido de indivíduos, ao passo que o número de parâmetros a serem estimados aumenta.

\subsection{Censuras}

Consideramos agora, nas amostras replicadas, situações em que são observadas ocorrências de censuras aleatórias para as transições $3 \rightarrow 4$ e $4 \rightarrow 5$, a fim de simular situações encontradas nos dados originais.

Nos modelos multi-estados, quando dizemos que um indivíduo foi censurado significa que, na última vez em que ele foi observado, permanecia no mesmo estado que estava quando observado anteriormente. Por exemplo, se um indivíduo foi censurado no estado 3, isso quer dizer que, na penúltima vez em que foi observado, ele estava no estado 3 e permaneceu lá até a última vez em que foi visto, e saiu do estudo por motivos alheios ao experimento, ou ainda que o estudo chegou ao final e o indivíduo ainda permanecia no estado 3, isto é, também não 
atingiu o estado absorvente.

$\mathrm{Na}$ situação com censuras aleatórias foram consideradas taxas de 15\%, 30\% e 50\%. Os indivíduos censurados para a transição $3 \rightarrow 4$ foram aleatoriamente selecionados e automaticamente não executam a transição $4 \rightarrow 5$. Em seguida, da mesma forma, selecionamos indivíduos que são censurados para a transição $4 \rightarrow 5$ e obtemos assim o total desejado de indivíduos censurados. Baseados nesses dados simulados obtemos os resultados apresentados a seguir.

\subsection{1 $15 \%$ de censuras}

Estimativas de $\beta_{k}^{\prime} s$ e respectivos erros padrão, com cinco e dez intervalos são apresentados nas tabelas a seguir, quando são observadas $15 \%$ de censuras.

Tabela 4.11: Estimativas de $\beta_{k}$ e respectivos erros padrão, com cinco intervalos e $15 \%$ de censuras.

\begin{tabular}{cccccc}
\hline$k$ & 1 & 2 & 3 & 4 & 5 \\
\hline$I_{k}$ & {$[0,00 ; 6,37)$} & {$[6,37 ; 9,02)$} & {$[9,02 ; 11,42)$} & {$[11,42 ; 13,58)$} & {$[13,58 ; 22,25]$} \\
$\beta_{k}$ & 0,057 & $-0,093$ & $-0,415$ & $-0,401$ & $-0,898$ \\
e.p. & 0,105 & 0,098 & 0,098 & 0,104 & 0,119 \\
\hline
\end{tabular}

Tabela 4.12: Estimativas de $\beta_{k}$ e respectivos erros padrão, com dez intervalos e $15 \%$ de censuras.

\begin{tabular}{cccccc}
\hline$k$ & 1 & 2 & 3 & 4 & 5 \\
\hline$I_{k}$ & {$[0,00 ; 5,78)$} & {$[5,78 ; 6,37)$} & {$[6,37 ; 7,74)$} & {$[7,74 ; 9,01)$} & {$[9,01 ; 10,44)$} \\
$\beta_{k}$ & $-0,043$ & 0,128 & $-0,023$ & $-0,161$ & $-0,502$ \\
e.p. & 0,163 & 0,138 & 0,139 & 0,137 & 0,137 \\
\hline \hline$k$ & 6 & 7 & 8 & 9 & 10 \\
\hline$I_{k}$ & {$[10,44 ; 11,42)$} & {$[11,42 ; 12,57)$} & {$[12,57 ; 13,58)$} & {$[13,58 ; 14,48)$} & {$[14,48 ; 22,25]$} \\
$\beta_{k}$ & $-0,321$ & $-0,362$ & $-0,440$ & $-0,872$ & $-0,94$ \\
e.p. & 0,142 & 0,147 & 0,147 & 0,150 & 0,193 \\
\hline
\end{tabular}

Os gráficos das Figuras 4.10 e 4.11 mostram a função $\beta(t)$ estimada pelo método do histograma crivo com taxa de $15 \%$ de censuras. 


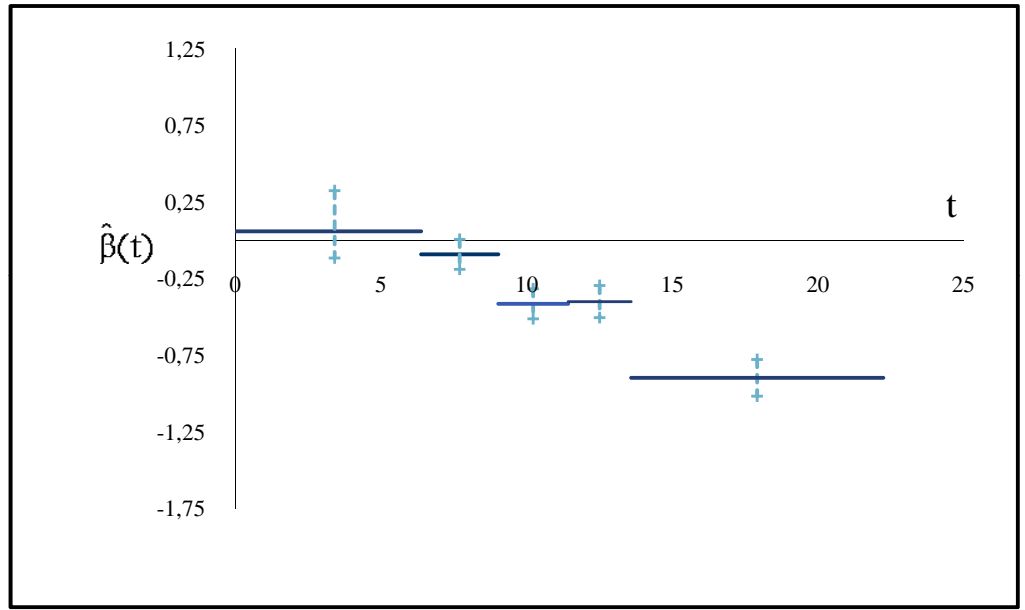

Figura 4.10: Estimativas de $\beta_{k}^{\prime} s$ com cinco intervalos, respectivos erros padrão e $15 \%$ de censuras.

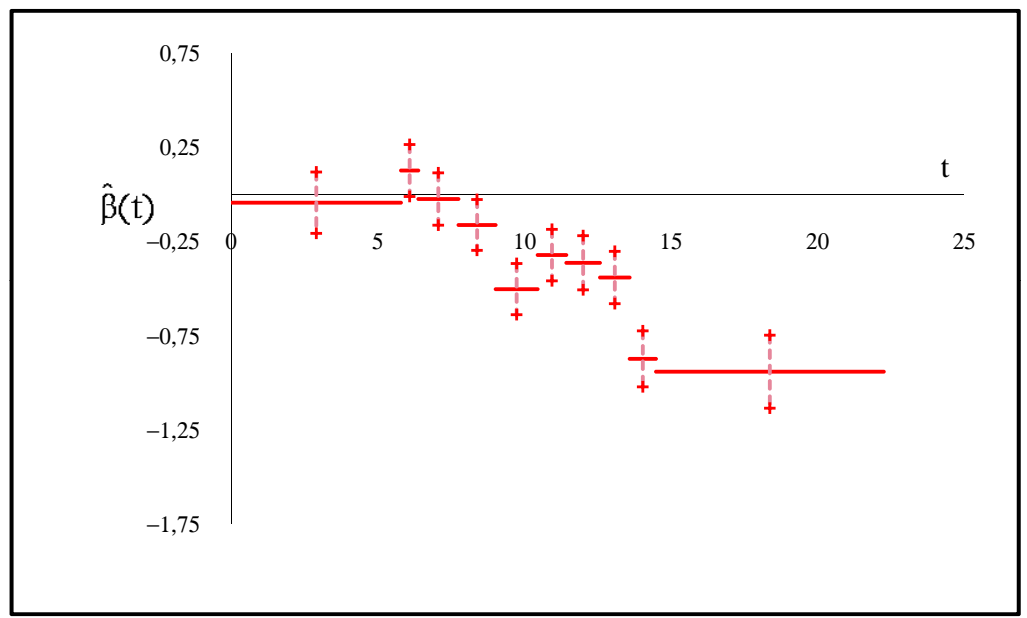

Figura 4.11: Estimativas de $\beta_{k}^{\prime} s$ com dez intervalos, respectivos erros padrão e $15 \%$ de censuras.

\subsection{2 $30 \%$ de censuras}

Estimativas de $\beta_{k}^{\prime} s$ e respectivos erros padrão, com cinco e dez intervalos são apresentados nas tabelas a seguir quando são observadas $30 \%$ de censuras. 
Tabela 4.13: Estimativas de $\beta_{k}$ e respectivos erros padrão, com cinco intervalos e $30 \%$ de censuras.

\begin{tabular}{cccccc}
\hline$k$ & 1 & 2 & 3 & 4 & 5 \\
\hline$I_{k}$ & {$[0,00 ; 6,28)$} & {$[6,28 ; 8,89)$} & {$[8,89 ; 11,25)$} & {$[11,25 ; 13,39)$} & {$[13,39 ; 22,24)$} \\
$\beta_{k}$ & 0,042 & 0,017 & $-0,474$ & $-0,428$ & $-1,045$ \\
e.p. & 0,107 & 0,100 & 0,103 & 0,107 & 0,125 \\
\hline
\end{tabular}

Tabela 4.14: Estimativas de $\beta_{k}$ e respectivos erros padrão, com dez intervalos e $30 \%$ de censuras.

\begin{tabular}{cccccc}
\hline$k$ & 1 & 2 & 3 & 4 & 5 \\
\hline$I_{k}$ & {$[0,00 ; 5,55)$} & {$[5,55 ; 6,28)$} & {$[6,28 ; 7,62)$} & {$[7,62 ; 8,89)$} & {$[8,89 ; 10,22)$} \\
$\beta_{k}$ & $-0,005$ & 0,076 & $-0,011$ & $-0,022$ & $-0,383$ \\
e.p. & 0,166 & 0,140 & 0,142 & 0,142 & 0,145 \\
\hline \hline$k$ & 6 & 7 & 8 & 9 & 10 \\
\hline$I_{k}$ & {$[10,22 ; 11,25)$} & {$[11,25 ; 12,29)$} & {$[12,29 ; 13,39)$} & {$[13,39 ; 14,36)$} & {$[14,36 ; 22,24]$} \\
$\beta_{k}$ & $-0,566$ & $-0,510$ & $-0,351$ & $-0,998$ & $-1,129$ \\
e.p. & 0,147 & 0,155 & 0,150 & 0,155 & 0,207 \\
\hline
\end{tabular}

Os gráficos das Figuras 4.12 e 4.13 mostram a função $\beta(t)$ estimada pelo método do histograma crivo com $30 \%$ de censuras.

\subsection{3 $50 \%$ de censuras}

Estimativas de $\beta_{k}^{\prime} s$ e respectivos erros padrão, com cinco e dez intervalos são apresentados nas Tabelas 4.15 e 4.16, respectivamente, quando são observadas $50 \%$ de censuras.

Tabela 4.15: Estimativas de $\beta_{k}$ e respectivos erros padrão, com cinco intervalos e $50 \%$ de censuras.

\begin{tabular}{cccccc}
\hline$k$ & 1 & 2 & 3 & 4 & 5 \\
\hline$I_{k}$ & {$[0,00 ; 6,25)$} & {$[6,25 ; 8,81)$} & {$[8,81 ; 11,04)$} & {$[11,04 ; 13,11)$} & {$[13,11 ; 22,25]$} \\
$\beta_{k}$ & 0,044 & 0,021 & $-0,410$ & $-0,372$ & $-1,155$ \\
e.p. & 0,109 & 0,102 & 0,110 & 0,118 & 0,130 \\
\hline
\end{tabular}

Os gráficos das Figuras 4.14 e 4.15 mostram a função $\beta(t)$ estimada pelo método do histo- 


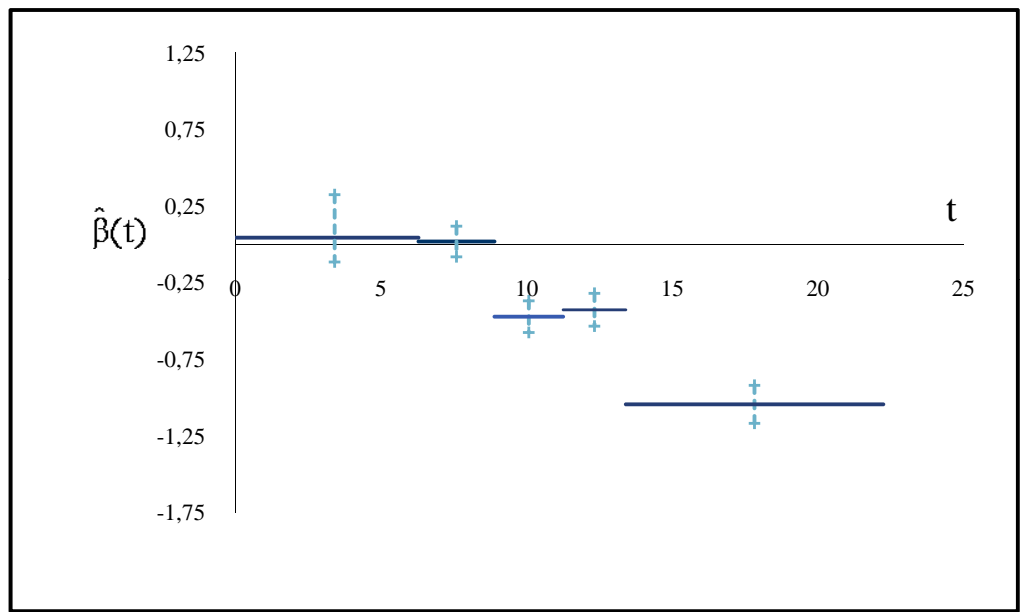

Figura 4.12: Estimativas de $\beta_{k}^{\prime} s$ com cinco intervalos, respectivos erros padrão e $30 \%$ de censuras.

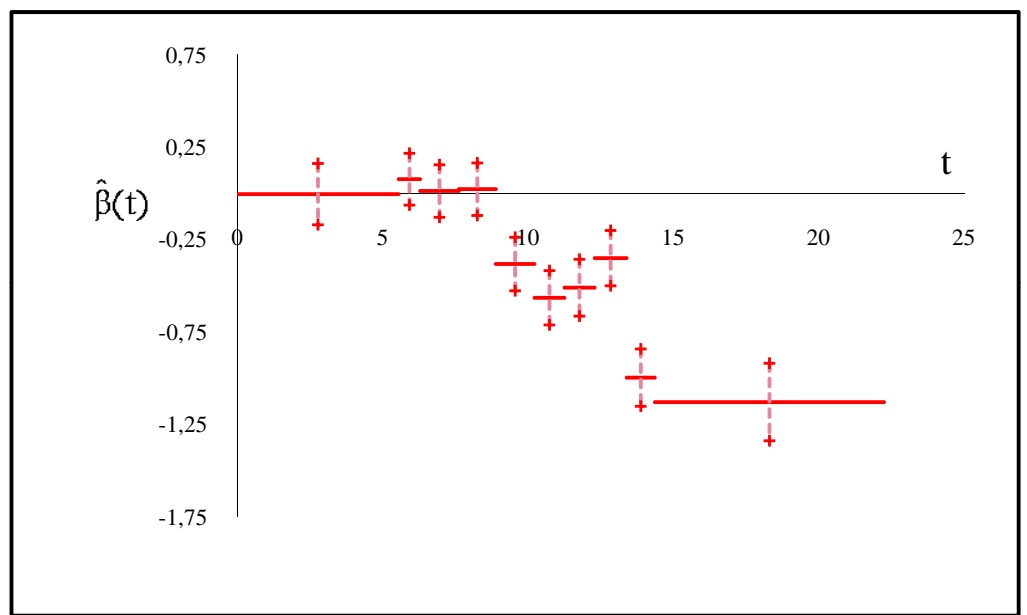

Figura 4.13: Estimativas de $\beta_{k}^{\prime} s$ com dez intervalos, respectivos erros padrão e $30 \%$ de censuras.

grama crivo. Como as amostras com censuras foram constituídas de forma a obter censuras apenas para as transições $3 \rightarrow 4$ e $4 \rightarrow 5$, que ocorrem em tempos posteriores, obtemos aumento dos erros padrão nos intervalos correspondentes. Comparando os gráficos, notamos que 
Tabela 4.16: Estimativas de $\beta_{k}$ e respectivos erros padrão, com dez intervalos e $50 \%$ de censuras.

\begin{tabular}{cccccc}
\hline$k$ & 1 & 2 & 3 & 4 & 5 \\
\hline$I_{k}$ & {$[0,00 ; 5,55)$} & {$[5,55 ; 6,24)$} & {$[6,24 ; 7,53)$} & {$[7,53 ; 8,81)$} & {$[8,81 ; 9,84)$} \\
$\beta_{k}$ & $-0,084$ & 0,139 & $-0,090$ & 0,133 & $-0,349$ \\
e.p. & 0,169 & 0,142 & 0,141 & 0,147 & 0,150 \\
\hline \hline$k$ & 6 & 7 & 8 & 9 & 10 \\
\hline$I_{k}$ & {$[9,84 ; 11,01)$} & {$[11,01 ; 12,02)$} & {$[12,02 ; 13,08)$} & {$[13,08 ; 14,25)$} & {$[14,25 ; 22,25]$} \\
$\beta_{k}$ & $-0,510$ & $-0,566$ & $-0,146$ & $-0,941$ & $-1,415$ \\
e.p. & 0,163 & 0,159 & 0,178 & 0,163 & 0,209 \\
\hline
\end{tabular}

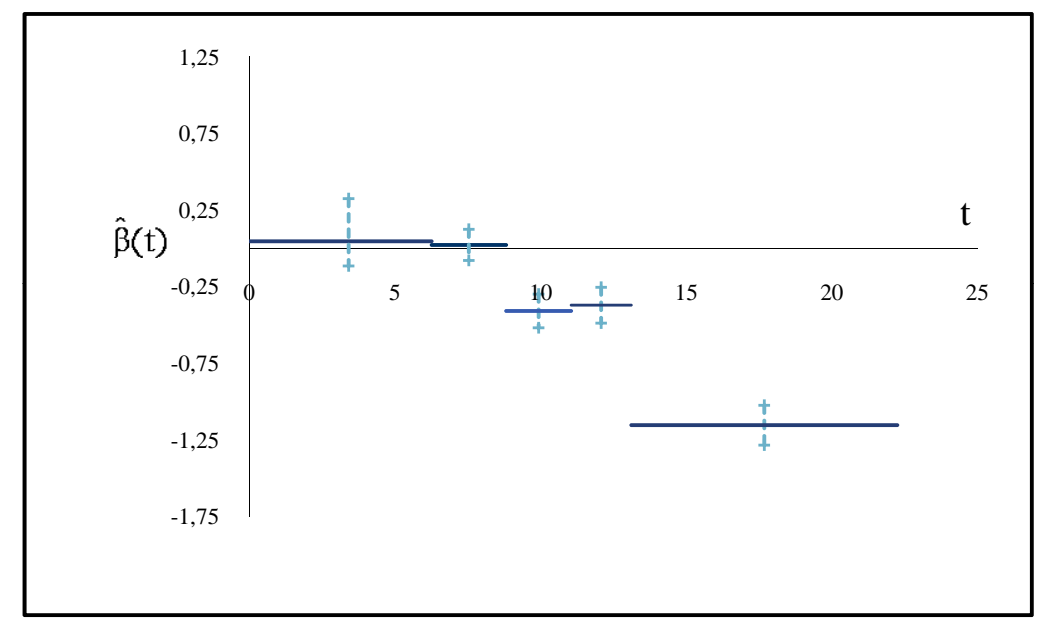

Figura 4.14: Estimativas de $\beta_{k}^{\prime} s$ com cinco intervalos, respectivos erros padrão e $50 \%$ de censuras.

o aumento da taxa de censura gerou aumento da instabilidade dos valores das estimativas de $\beta_{k}^{\prime} s$, o que é esperado. 


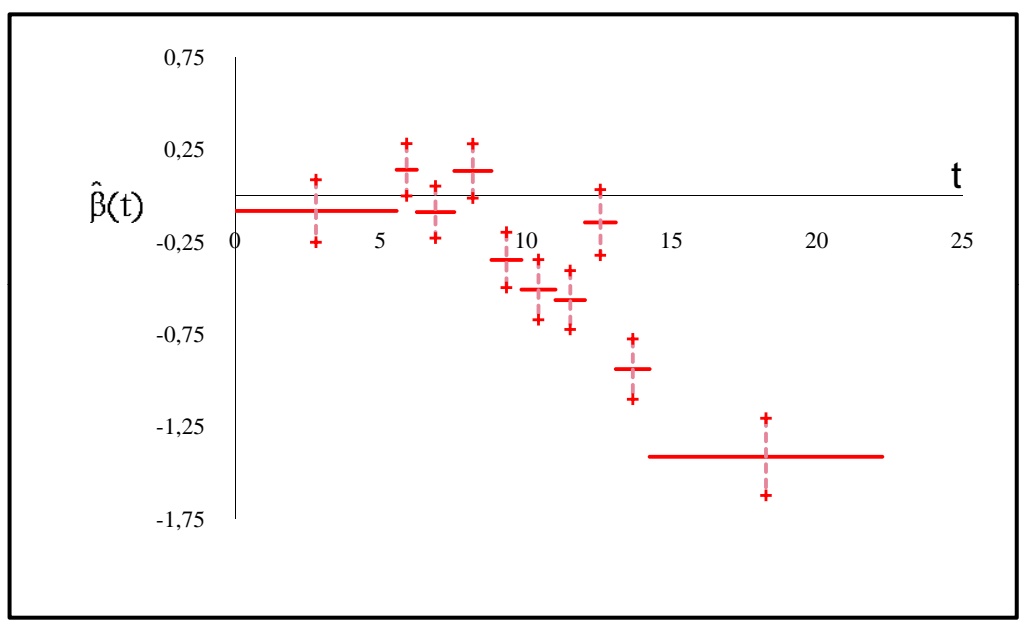

Figura 4.15: Estimativas de $\beta_{k}^{\prime} s$ com dez intervalos, respectivos erros padrão e $50 \%$ de censuras. 


\section{Capítulo 5}

\section{Considerações finais}

Quando trabalhamos com dados longitudinais, a primeira idéia é usar os modelos de efeitos aleatórios. Com o presente trabalho não foi diferente. Inicialmente, pensou-se na modelagem conjunta dos tempos e dos escores observados para obter estimativas mais eficientes dos parâmetros do modelo considerado, entretanto, essa modelagem é mais adequada quando as saídas observadas durante o experimento são causadas por censuras informativas (Hogan \& Laird, 1997), o que não ocorre neste caso.

Os modelos multi-estados, além de descrever a evolução das respostas associadas com unidades experimentais observados longitudinalmente, não supõem nenhum modelo que relacione tempos e escores. Neles, uma das respostas de interesse (escores) é categorizada formando os estados que são fixas e modelamos os tempos de transição ou as intensidades de transição entre os estados.

Evidentemente, essa modelagem também possui algumas restrições. O número de estados, por exemplo, não pode ser muito grande devido ao alto número de parâmetros a serem estimados. Além disso, as estruturas de ligação entre os estados devem estar bem-definidas para descrever o problema em questão.

Neste trabalho apresentamos um modelo multi-estados não-homogêneo, em que a intensidade de transição envolve covariáveis cujos efeitos podem se alterar com o tempo. Na abor- 
dagem considerada, efeito de grupo, representado pelo coeficiente $\beta(t)$ é compartilhado por todas as transições. Entretanto, para o conjunto de dados que motivou este trabalho, temos um processo progressivo, em que uma vez atingido certo estado não é possível visitar os estados anteriores novamente, de tal forma que a modelagem considerada se encaixa naturalmente.

Para encontrar a estimativa da matriz $\boldsymbol{\Lambda}(t)$ em (2.1) para o caso não homogêneo, é necessário estimar o valor da intensidade de transição $\lambda_{i j}(t)$ e, para isso, é preciso estimar a função de risco basal $\alpha_{0 h j}(t)$ para cada transição $h \rightarrow j$ conjuntamente com $\beta(t)$. Isso é possível se considerarmos a função de verossimilhança completa.

Hougaard (2000) deriva a função de verossimilhança completa para modelos multi-estados markovianos. Ele considera $H$ estados, numerados de 1 a $H$, e a função de verossimilhança é baseada em um modelo progressivo, isto é, supõe que o número atual de transições e a ordem em que ocorrem são conhecidos. De acordo com a estrutura de dados, alguns dos termos são considerados identicamente iguais a zero. Se a intensidade de transição do estado $h$ para o estado $j$ é denotada por $\lambda_{h j}(t)$, o risco total no estado $h$ é $\sum_{h \neq j} \lambda_{h j}(t)$, simplesmente denotado por $\lambda_{h}(t)$. Cada unidade experimental é acompanhada por um período de tempo de 0 a $C$ em que $E$ eventos são observados. O processo inicia-se em $s_{0}$ em $T_{0}=0$. O $m$-ésimo evento é uma transição que passa pelo estado $s_{m}$, no tempo $T_{m}, m=1, \ldots, E$. Então, a função de verossimilhança é

$$
\prod_{m=1}^{E} \lambda_{s_{m-1} s_{m}}\left(T_{m}\right) \exp \left\{-\int_{T_{m-1}}^{T_{m}} \lambda_{s_{m-1}}(u) \mathrm{d} u\right\} \exp \left\{-\int_{T_{E}}^{C} \lambda_{s_{E}}(u) \mathrm{d} u\right\}
$$

A função de verossimilhança pode ser escrita como produto de todas as transições porque a propriedade markoviana permite que toda a história do processo seja esquecida. A transição futura depende apenas do estado atual. Como os dois termos exponenciais em (5.1) são muito similares, Hougaard (2000) introduz uma notação $Q=E+1, T_{Q}=C, S_{Q}=0$, no caso de censura (e $Q=E$ em caso de evento no tempo $C$ ), e assim reformula a verossimilhança como

$$
\prod_{m=1}^{Q} \lambda_{s_{m-1} s_{m}}\left(T_{m}\right)^{\mathbb{I}\left\{s_{m} \neq 0\right\}} \exp \left\{-\int_{T_{m-1}}^{T_{m}} \lambda_{s_{m-1}}(u) \mathrm{d} u\right\}
$$


Essa verossimilhança diz que cada transição é uma contribuição que corresponde à função de risco naquele tempo e que, para os períodos sem transição, há termos exponenciais com a integral do risco experimentado pelo processo. No modelo markoviano, a verossimilhança pode ser reformulada nessa linha. A vantagem disso é que podemos colecionar termos a partir de diferentes pacientes e repetidas ocorrências de uma mesma transição em um simples paciente. Para fazê-lo, Hougaard (2000) introduz indicadores $D_{h j m}=\mathbb{I}\left\{s_{m-1}=h, s_{m}=j\right\}$, que assumem valor 1 quando a $m$-ésima transição vai do estado $h$ para o estado $j$, e a função do conjunto de risco $R_{h}(t)$, que assume valor 1 quando o processo está em $h$ no tempo $t^{-}$.

A fórmula para essa função de risco é

$$
R_{h}(t)=\sum_{m=1}^{Q} \mathbb{I}\left\{s_{m-1}=h, T_{m-1}<t<T_{m}\right\}
$$

Então,

$$
\prod_{h} \prod_{j}\left[\left\{\prod_{m=1}^{Q} \lambda_{h j}\left(T_{m}\right)^{D_{h j m}}\right\} \exp \left\{-\int_{0}^{C} R_{h}(u) \lambda_{h j}(u) \mathrm{d} u\right\}\right], \quad h, j=1, \ldots, H
$$

descreve a verossimilhança como um produto sobre todas as possíveis transições, e cada termo envolve somente a função de transição para uma particular transição.

Se considerarmos cada transição $h \rightarrow j$ como um evento, a verossimilhança (5.2) corresponde ao produto de verossimilhanças completas usuais em análise de sobrevivência.

Quando trabalhamos com a verossimilhança completa, nos modelos multi-estados, aumentamos o número de parâmetros a serem estimados. Para se ter uma idéia, sob o modelo (2.16), usado na aplicação da Seção 4.2 (o estimador histograma crivo com cinco partições para estimar $\beta(t)$ e $\alpha_{0 h j}(t)$ para cada transição $\left.h \rightarrow j\right)$, teríamos um total de 25 parâmetros em vez dos cinco iniciais estimados na Seção 4.2. Um aumento considerável de parâmetros, o que demanda tempo de processamento, além de maior instabilidade dos valores estimados.

Uma vez ajustado o modelo com os coeficientes dependentes do tempo, devemos verificar a qualidade de ajuste do modelo proposto, que não foi apresentada neste trabalho. Um ponto 
inicial seria considerar os estudos de Marzec \& Marzec (1997) e Murphy \& Sen (1991), que produzem teste de qualidade de ajuste para o modelo (2.15) de Murphy \& Sen (1991).

Mais recentemente, Scheike \& Zhang (2007) mostram como tornar o modelo de regressão mais flexível com a inclusão de efeitos da covariável dependente do tempo através dos modelos lineares generalizados. Vários trabalhos têm surgido na área de modelos multi-estados. Entre eles estão os modelos não markovianos que levam em conta toda a história do processo, mas esse é um tema para um trabalho futuro. 


\section{Apêndice A}

\section{Lemas}

\section{A.1 Lemas}

Lema A.1. Se as suposições $A(1), A(3), C(1), D(1)$ acontecem e se $\lim _{n}\|l\|=0$, quando $n \rightarrow \infty$, temos

(1)

$$
\max _{1 \leq i \leq K}\left|\int_{0}^{\tau} \mathbb{I}_{k}(u) d \bar{M}_{h}(u)\right|=O_{p}(1 / \sqrt{n}) \quad e
$$

(2)

$$
\begin{array}{r}
\sup _{1 \leq u \leq \tau}\left|\frac{S_{h}^{(i)}(\bar{\beta}, u)}{S_{h}^{(0)}(\bar{\beta}, u)}-\frac{S_{h}^{(i)}\left(\beta_{0}, u\right)}{S_{h}^{(0)}\left(\beta_{0}, u\right)}\right|=O_{p}\left(l_{(K)}\right), \quad i=1,2, \\
\text { em que } \bar{M}_{h}(u)=n^{-1} \sum_{j=1}^{n} M_{j h}(u) \text { para } u \in[0, \tau] \quad, s=1, \ldots, S-1 .
\end{array}
$$

Lema A.2. Se as suposições $A, C(1), D(1)$ acontecem, $\lim _{n}\|l\|=0$ e $\lim \sup \left(l_{(K)} / l_{(1)}\right)<\infty$, então, quando $n \rightarrow \infty$, 
(1)

$$
\begin{aligned}
& \sum_{k=1}^{K}\left(\left(n l_{k}\right)^{-1} \frac{\partial}{\partial \beta_{k}} \mathcal{L}_{n h}\left(\bar{\beta}^{n}\right)\right)^{2} \\
\leq & 2\left(\|l\|^{4} n^{-1}\right)\left[\|l\|^{4} \int_{0}^{\tau} \sum_{k=1}^{K} \mathbb{I}_{k}(u) l_{k}^{-2} v_{h}\left(\beta_{0}, u\right) s_{h}^{(0)}\left(\beta_{0}, u\right) \alpha_{0 h}(u) d u+o_{p}(1)\right] \\
& +O_{p}\left(\|l\|^{6}\right)+O_{p}(1 / n),
\end{aligned}
$$

(2)

$$
\max _{1 \leq k \leq K}\left|\left(n l_{k}\right)^{-1} \frac{\partial^{2}}{\partial \beta_{k}^{2}} \mathcal{L}_{n h}\left(\bar{\beta}^{n}\right)+l_{k}^{-1} \sigma_{k}^{2}\right|=O_{p}\left(\left(\sqrt{n}\|l\|^{2}\right)^{-1}\right)+o_{p}(1) \quad e
$$

(3)

$$
\max _{1 \leq k \leq K} \sup _{\substack{\beta^{*} \in \Theta_{K} \\ \beta^{*}-\bar{\beta}^{n} \|<0,5 \gamma}}\left|\left(n l_{k}\right)^{-1} \frac{\partial^{3}}{\partial \beta_{k}^{3}} \mathcal{L}_{n h}\left(\beta^{*}\right)\right|=O_{p}\left(\left(\sqrt{n}\|l\|^{2}\right)^{-1}\right)+O_{p}(1)
$$

Lema A.3. Se as suposições $C(1), D(1)$ acontecem, $\lim _{n}\|l\|=0$ e $\lim \sup _{n}\left(l_{(K)} / l_{(1)}\right)<\infty$,

$$
\sum_{k=1}^{K}\left(\sum_{h=1}^{H-1} \sigma_{k h}^{2}-\sum_{h=1}^{H-1} \widehat{\sigma}_{k h}^{2}\right)^{2}=\|l\|^{4}\left[O_{p}\left(\frac{1}{\|l\|^{4} n}\right)+O_{p}\left(\|l\|^{2}\right)+O\left(\left\|\widehat{\beta}^{n}-\bar{\beta}^{n}\right\|^{2}\right)\right] .
$$

Lema A.4. Se as suposições $A, C, D$ acontecem, $\lim _{n}\|l\|=0$ e $\lim \sup _{n}\left(l_{(K)} / l_{(1)}\right)<\infty$, então, quando $n \rightarrow \infty$,

$$
\begin{aligned}
& \sup _{0 \leq t \leq \tau}\left|\sqrt{n} \int_{0}^{t}\left[\sum_{k=1}^{K} \mathbb{I}_{k}(u) \frac{l_{k}}{\sum_{h=1}^{H-1} \sigma_{k h}^{2}}\right] \sum_{h=1}^{H-1}\left(\mathbb{E}_{h}\left(\beta_{0}, u\right)-\mathbb{E}_{h}\left(\bar{\beta}^{n}, u\right)\right) S_{h}^{(0)}\left(\beta_{0}, u\right) \alpha_{0 h}(u) d u\right| \\
= & O_{p}\left(\sqrt{n}\|l\|^{4}\right)+O_{p}\left(\|l\|^{2}\right) .
\end{aligned}
$$

\section{Demonstrações}


As demonstrações dos Lemas A.1 e A.2 são encontradas em Murphy \& Sen (1991). Os Lemas A.3 e A.4 possuem algumas modificações e as demonstrações são apresentadas a seguir.

\section{Demonstração do Lema A.3}

Note que

$$
\begin{aligned}
& \sum_{k=1}^{K}\left(\sum_{h=1}^{H-1} \sigma_{k h}^{2}-\sum_{h=1}^{H-1} \widehat{\sigma}_{k h}^{2}\right)^{2} \\
= & \sum_{k=1}^{K}\left(\sum_{h=1}^{H-1} \int_{0}^{\tau} \mathbb{I}_{k}(u) v_{h}\left(\beta_{0}, u\right) s_{h}^{(0)}\left(\beta_{0}, u\right) \alpha_{0 h}(u) \mathrm{d} u+\sum_{h=1}^{H-1} \int_{0}^{\tau} \mathbb{I}_{k}(u) \mathbb{V}_{h}(\bar{\beta}, u) \mathrm{d} \bar{N}_{h}(u)\right. \\
& \left.+\sum_{h=1}^{H-1} \frac{1}{2 n} \frac{\partial^{3}}{\partial \beta_{k}^{3}} \mathcal{L}_{n h}\left(\beta^{*}\right)\left(\widehat{\beta}_{k}^{n}-\bar{\beta}_{k}^{n}\right)\right)^{2} \\
& \text { em que }\left\|\beta^{*}-\widehat{\beta}^{n}\right\| \leq\left\|\bar{\beta}^{n}-\widehat{\beta}^{n}\right\| \\
\leq & 2 \sum_{k=1}^{K}\left(\sum_{h=1}^{H-1} \int_{0}^{\tau} \mathbb{I}_{k}(u) v_{h}\left(\beta_{0}, u\right) s_{h}^{(0)}\left(\beta_{0}, u\right) \alpha_{0 h}(u) \mathrm{d} u+\sum_{h=1}^{H-1} \int_{0}^{\tau} \mathbb{V}_{h}(\bar{\beta}, u) \mathrm{d} \bar{N}_{h}(u)\right)^{2} \\
& +2 \sum_{k=1}^{K}\left(\sum_{h=1}^{H-1} \frac{1}{2 n} \frac{\partial^{3}}{\partial \beta_{k}^{3}} \mathcal{L}_{n h}\left(\beta^{*}\right)\left(\widehat{\beta}_{k}^{n}-\bar{\beta}_{k}^{n}\right)\right)^{2} .
\end{aligned}
$$

Com relação ao termo (A.9), temos que

$$
\begin{aligned}
& 2 \sum_{k=1}^{K}\left(\sum_{h=1}^{H-1} \frac{1}{2 n} \frac{\partial^{3}}{\partial \beta^{3}} \mathcal{L}_{n h}\left(\beta^{*}\right)\left(\widehat{\beta}_{k}^{n}-\bar{\beta}_{k}^{n}\right)\right)^{2} \\
= & 2 \sum_{k=1}^{K}\left(\sum_{h=1}^{H-1} \frac{1}{2 n} \frac{l_{k}}{l_{k}} \frac{\partial^{3}}{\partial \beta^{3}} \mathcal{L}_{n h}\left(\beta^{*}\right)\left(\widehat{\beta}_{k}^{n}-\bar{\beta}_{k}^{n}\right)\right)^{2} \\
\leq & 2 \sum_{k=1}^{K} \sum_{h=1}^{H-1}\left(\frac{l_{k}}{2 n}\left(\widehat{\beta}_{k}^{n}-\bar{\beta}_{k}^{n}\right)\right)^{2} \sum_{k=1}^{K} \sum_{h=1}^{H-1}\left(\frac{\partial^{3}}{\partial \beta_{k}^{3}} \mathcal{L}_{n h}\left(\beta^{*}\right)\right)^{2} \\
\leq & \frac{1}{2} l_{(K)}^{2} \sum_{h=1}^{H-1} \sum_{k=1}^{K}\left(\widehat{\beta}_{k}^{n}-\bar{\beta}_{k}^{n}\right)^{2} \sum_{k=1}^{K}\left(\sum_{h=1}^{H-1} \frac{1}{n l_{k}} \frac{\partial^{3}}{\partial \beta^{3}} \mathcal{L}_{n h}\left(\beta^{*}\right)\right)^{2}
\end{aligned}
$$




$$
\begin{aligned}
& \leq \frac{\|l\|^{4}}{2} \sum_{h=1}^{H-1}\left\|\widehat{\beta}^{n}-\bar{\beta}^{n}\right\|^{2} \sum_{h=1}^{H-1}\left(\max _{1 \leq k \leq K} \sup _{\substack{\beta^{*} \in \Theta_{K} \\
\left\|\beta^{*}-\bar{\beta}^{n}\right\|<0,5 \gamma}}\left|\sum_{h=1}^{H-1}\left(n l_{k}\right)^{-1} \frac{\partial^{3}}{\partial \beta^{3}} \mathcal{L}_{n h}\left(\beta^{*}\right)\right|\right)^{2} \\
& \leq \frac{\|l\|^{4}}{2}\left\|\widehat{\beta}^{n}-\bar{\beta}^{n}\right\|^{2}\left\{(H-1)\left[O_{p}\left(\sqrt{n}\|l\|^{-2}\right)+O_{p}(1)\right]\right\}^{2} \\
& \text { (pelos resultados do Lema A.2 } \\
& \leq \frac{\|l\|^{4}}{2}\left\|\widehat{\beta}^{n}-\bar{\beta}^{n}\right\|^{2}\left\{O_{p}(1)\right\}=O_{p}\left(\|l\|^{4}\right)\left\|\widehat{\beta}^{n}-\bar{\beta}^{n}\right\|^{2} \text {. }
\end{aligned}
$$

Com relação ao termo A.8 do Lema A.3.

$$
\begin{aligned}
& 2 \sum_{k=1}^{K}\left(\sum_{h=1}^{H-1} \int_{0}^{\tau} \mathbb{I}_{k}(u) v_{h}\left(\beta_{0}, u\right) s_{h}^{(0)}\left(\beta_{0}, u\right) \alpha_{0 h}(u) \mathrm{d} u+\sum_{h=1}^{H-1} \int_{0}^{\tau} \mathbb{V}_{h}(\bar{\beta}, u) \mathrm{d} \bar{N}_{h}(u)\right)^{2} \\
= & 2 \sum_{k=1}^{K}\left(\sum_{h=1}^{H-1} \int_{0}^{\tau} \mathbb{I}_{k}(u) v_{h}\left(\beta_{0}, u\right) s_{h}^{(0)}\left(\beta_{0}, u\right) \alpha_{0 h}(u) \mathrm{d} u\right. \\
& \left.+\sum_{h=1}^{H-1} \int_{0}^{\tau} \mathbb{V}_{h}(\bar{\beta}, u) \mathrm{d} \bar{M}_{h}(u)+\sum_{h=1}^{H-1} \int_{0}^{\tau} \mathbb{V}_{h}(\bar{\beta}, u) \mathrm{d} \bar{\Lambda}_{h}(u)\right)^{2} \\
= & 2 \sum_{k=1}^{K}\left(l_{k}^{-1} \sum_{h=1}^{H-1} \int_{0}^{\tau} \mathbb{I}_{k}(u) v_{h}\left(\beta_{0}, u\right) s_{h}^{(0)}\left(\beta_{0}, u\right) \alpha_{0 h}(u) \mathrm{d} u\right. \\
& \left.+\sum_{h=1}^{H-1} \int_{0}^{\tau} l_{k}^{-1} \mathbb{V}_{h}(\bar{\beta}, u) \mathrm{d} \bar{M}_{h}(u)+\sum_{h=1}^{H-1} \int_{0}^{\tau} l_{k}^{-1} \mathbb{V}_{h}(\bar{\beta}, u) \mathrm{d} \bar{\Lambda}_{h}(u)\right)^{2} l_{k}^{2} \\
\leq & 4 \sum_{k=1}^{K}\left[\sum_{h=1}^{H-1} \int_{0}^{\tau} l_{k}^{-1} \mathbb{V}_{h}(\bar{\beta}, u) \mathrm{d} \bar{M}_{h}(u)\right]^{2}\|\|^{4} \\
& +4 \sum_{k=1}^{K}\left[\left(l_{k}^{-1} \sum_{h=1}^{H-1} \int_{0}^{\tau} \mathbb{I}_{k}(u) v_{h}\left(\beta_{0}, u\right) s_{h}^{(0)}\left(\beta_{0}, u\right) \alpha_{0 h}(u) \mathrm{d} u\right.\right. \\
& \left.\left.+\sum_{h=1}^{H-1} \int_{0}^{\tau} l_{k}^{-1} \mathbb{V}_{h}(\bar{\beta}, u) \mathrm{d} \bar{\Lambda}_{h}(u)\right)\right]^{2}\|l\|^{4}
\end{aligned}
$$

Usando a desigualdade de Lenglart, Murphy \& Sen (1991) mostram que 


$$
\begin{aligned}
& 4 \sum_{k=1}^{K}\left[\int_{0}^{\tau} l_{k}^{-1} \mathbb{V}_{h}(\bar{\beta}, u) \mathrm{d} \bar{M}_{h}(u)\right]^{2} l_{k}^{2} \leq 4 \sum_{k=1}^{K}\left[\int_{0}^{\tau} l_{k}^{-1} \mathbb{V}_{h}(\bar{\beta}, u) \mathrm{d} \bar{M}_{h}(u)\right]^{2} O\left(\|l\|^{4}\right) \\
= & O\left(\frac{1}{n\|l\|^{4}}\right) O\left(\|l\|^{4}\right) .
\end{aligned}
$$

Logo, A.10 pode ser escrito como

$$
\begin{aligned}
& 4 \sum_{k=1}^{K}\left[\sum_{h=1}^{H-1} \int_{0}^{\tau} l_{k}^{-1} \mathbb{V}_{h}(\bar{\beta}, u) \mathrm{d} \bar{M}_{h}(u)\right]^{2}\|l\|^{4} \\
\leq & 4 \sum_{k=1}^{K}\left[\sum_{h=1}^{H-1}\left[\int_{0}^{\tau} l_{k}^{-1} \mathbb{V}_{h}(\bar{\beta}, u) \mathrm{d} \bar{M}_{h}(u)\right]^{2} \sum_{h=1}^{H-1} 1^{2}\right]\|l\|^{4} \\
= & 4 \sum_{k=1}^{K} \sum_{h=1}^{H-1}\left[\int_{0}^{\tau} l_{k}^{-1} \mathbb{V}_{h}(\bar{\beta}, u) \mathrm{d} \bar{M}_{h}(u)\right]^{2}(H-1)\|l\|^{4} \\
= & (H-1) O\left(\frac{1}{n\|l\|^{4}}\right) O\left(\|l\|^{4}\right)=O\left(\frac{1}{n\|l\|^{4}}\right) O\left(\|l\|^{4}\right) .
\end{aligned}
$$

E agora, somando e subtraindo a quantidade

$$
\sum_{h=1}^{H-1} l_{k}^{-1} \int_{0}^{\tau} \mathbb{I}_{k}(u) \mathbb{V}_{h}\left(\beta_{0}, u\right) S_{h}^{(0)}\left(\beta_{0}, u\right) \alpha_{0 h}(u) \mathrm{d} u
$$

ao termo A.11 do Lema A.3.

$$
\begin{aligned}
& 4 \sum_{k=1}^{K}\left[\left(-l_{k}^{-1} \sum_{h=1}^{H-1} \int_{0}^{\tau} \mathbb{I}_{k}(u) v_{h}\left(\beta_{0}, u\right) s_{h}^{(0)}\left(\beta_{0}, u\right) \alpha_{0 h}(u) \mathrm{d} u\right.\right. \\
& \left.\left.+\sum_{h=1}^{H-1} \int_{0}^{\tau} l_{k}^{-1} \mathbb{V}_{h}(\bar{\beta}, u) \mathrm{d} \bar{\Lambda}_{h}(u)\right)\right]^{2}\|l\|^{4}
\end{aligned}
$$




$$
\begin{aligned}
= & \sum_{k=1}^{K}\left[l_{k}^{-1} \sum_{h=1}^{H-1} \int_{0}^{\tau} \mathbb{I}_{k}(u) v_{h}\left(\beta_{0}, u\right) s_{h}^{(0)}\left(\beta_{0}, u\right) \alpha_{0 h}(u) \mathrm{d} u\right. \\
& +\sum_{h=1}^{H-1} \int_{0}^{\tau} l_{k}^{-1} \mathbb{V}_{h}(\bar{\beta}, u) S_{h}^{(0)}\left(\beta_{0}, u\right) \alpha_{0 h}(u) \mathrm{d} u \\
& +\sum_{h=1}^{H-1} l_{k}^{-1} \int_{0}^{\tau} \mathbb{I}_{k}(u) \mathbb{V}_{h}\left(\beta_{0}, u\right) S_{h}^{(0)}\left(\beta_{0}, u\right) \alpha_{0 h}(u) \mathrm{d} u \\
& \left.-\sum_{h=1}^{H-1} l_{k}^{-1} \int_{0}^{\tau} \mathbb{I}_{k}(u) \mathbb{V}_{h}\left(\beta_{0}, u\right) S_{h}^{(0)}\left(\beta_{0}, u\right) \alpha_{0 h}(u) \mathrm{d} u\right]^{2}\|l\|^{4} \\
\leq & \sum_{k=1}^{K}\left[l_{k}^{-1} \sum_{h=1}^{H-1} \int_{0}^{\tau} \mathbb{I}_{k}(u)\left[\mathbb{V}_{h}(\bar{\beta}, u)-\mathbb{V}_{h}\left(\beta_{0}, u\right)\right] S_{h}^{(0)}\left(\beta_{0}, u\right) \alpha_{0 h}(u) \mathrm{d} u\right]^{2}\|l\|^{4} \\
& +\sum_{k=1}^{K}\left[l _ { k } ^ { - 1 } \sum _ { h = 1 } ^ { H - 1 } \int _ { 0 } ^ { \tau } \mathbb { I } _ { k } ( u ) \left(\mathbb{V}_{h}\left(\beta_{0}, u\right) S_{h}^{(0)}\left(\beta_{0}, u\right)\right.\right. \\
& \left.\left.-v_{h}\left(\beta_{0}, u\right) s_{h}^{(0)}\left(\beta_{0}, u\right)\right) \alpha_{0 h}(u) \mathrm{d} u\right]_{\| l}^{2} l \|^{4}
\end{aligned}
$$

Pelo Lema A.1, A.12 é $O_{p}\left(\|l\|^{6}\right)$, pois

$$
\begin{aligned}
& \sum_{k=1}^{K}\left[l_{k}^{-1} \sum_{h=1}^{H-1} \int_{0}^{\tau} \mathbb{I}_{k}(u)\left[\mathbb{V}_{h}(\bar{\beta}, u)-\mathbb{V}_{h}\left(\beta_{0}, u\right)\right] S_{h}^{(0)}\left(\beta_{0}, u\right) \alpha_{0 h}(u) \mathrm{d} u\right]^{2}\|l\|^{4} \\
\leq & l_{(1)}^{-2} \sum_{k=1}^{K}\left\{\sum _ { h = 1 } ^ { H - 1 } \int _ { 0 } ^ { \tau } \mathbb { I } _ { k } ( u ) \left[\frac{S_{h}^{(1)}(\bar{\beta}, u)}{S_{h}^{(2)}(\bar{\beta}, u)}-\left(\frac{S_{h}^{(1)}(\bar{\beta}, u)}{S_{h}^{(0)}(\bar{\beta}, u)}\right)^{2}\right.\right. \\
& \left.\left.-\left[\frac{S_{h}^{(2)}\left(\beta_{0}, u\right)}{S_{h}^{(0)}\left(\beta_{0}, u\right)}-\left(\frac{S_{h}^{(1)}\left(\beta_{0}, u\right)}{S_{h}^{(0)}\left(\beta_{0}, u\right)}\right)^{2}\right]\right] S_{h}^{(0)}\left(\beta_{0}, u\right) \alpha_{0 h}(u) \mathrm{d} u\right\}^{2}\|l\|^{4} \\
\leq & \left(\frac{l_{(K)}}{l_{(1)}}\right)^{2} O_{p}(1) \sum_{k=1}^{K}\left[\sum_{h=1}^{H-1} \int_{0}^{\tau} \mathbb{I}_{k}(u) S_{h}^{(0)}\left(\beta_{0}, u\right) \alpha_{0 h}(u) \mathrm{d} u\right]^{2}\|l\|^{4}
\end{aligned}
$$




$$
\begin{aligned}
= & O_{p}(1) \sum_{k=1}^{K}\left[\sum_{h=1}^{H-1} \int_{0}^{\tau} \mathbb{I}_{k}(u)\left|S_{h}^{(0)}\left(\beta_{0}, u\right)-s_{h}^{(0)}\left(\beta_{0}, u\right)\right| \alpha_{0 h}(u) \mathrm{d} u\right. \\
& \left.+\sum_{h=1}^{H-1} \int_{0}^{\tau} \mathbb{I}_{k}(u) s_{h}^{(0)}\left(\beta_{0}, u\right) \alpha_{0 h}(u) \mathrm{d} u\right]^{2}\|l\|^{4} \\
= & O_{p}(1) \sum_{k=1}^{K}\left[\sum_{h=1}^{H-1} \int_{0}^{\tau} \mathbb{I}_{k}(u) \mathrm{d} u+\sum_{h=1}^{H-1} l_{k}\right]^{2}\|l\|^{4} \\
= & O_{p}(1) \sum_{k=1}^{K}\left[\sum_{h=1}^{H-1}\left(2 l_{k}\right)^{2}\right]\|l\|^{4} \\
= & O_{p}\left(\|l\|^{2}\right)\|l\|^{4} .
\end{aligned}
$$

Quanto ao termo A.13 do Lema A.3.

$$
\begin{aligned}
& \sum_{k=1}^{K}\left[l_{k}^{-1} \sum_{h=1}^{H-1} \int_{0}^{\tau} \mathbb{I}_{k}(u)\left(\mathbb{V}_{h}\left(\beta_{0}, u\right) S_{h}^{(0)}\left(\beta_{0}, u\right)-v_{h}\left(\beta_{0}, u\right) s_{h}^{(0)}\left(\beta_{0}, u\right)\right) \alpha_{0 h}(u) \mathrm{d} u\right]^{2}\|l\|^{4} \\
= & O(1) \sum_{k=1}^{K}\left[l_{k}^{-1} \sum_{h=1}^{H-1} \int_{0}^{\tau} \mathbb{I}_{k}(u) \mathrm{d} u\right]^{2}\|l\|^{4} \\
= & O(1) \sum_{k=1}^{K}\left[l_{k}^{-1} \sum_{h=1}^{H-1} l_{k}\right]^{2}\|l\|^{4} \\
= & O\left(\|l\|^{4}\right),
\end{aligned}
$$

de onde concluímos que

$$
\sum_{k=1}^{K}\left(\sum_{h=1}^{H-1} \sigma_{k h}^{2}-\sum_{h=1}^{H-1} \widehat{\sigma}_{k h}^{2}\right)=\|l\|^{4}\left[O_{p}\left(\frac{1}{n\|l\|^{4}}\right)+O_{p}\left(\|l\|^{2}\right)+O\left(\left\|\widehat{\beta}^{n}-\bar{\beta}^{n}\right\|^{2}\right)\right] .
$$

Da mesma forma que em Murphy \& Sen (1991), temos como conseqüência desse lema que

$$
P\left[\min \frac{\sum_{h=1}^{H-1} \widehat{\sigma}_{k h}^{2}}{l_{k}}>\frac{1}{2} L\right] \stackrel{p}{\rightarrow} 1
$$


pois

$$
\sigma_{k}^{2}=\sum_{h=1}^{H-1} \sigma_{k h}^{2}=\sum_{h=1}^{H-1} \int_{0}^{\tau} \mathbb{I}_{k}(u) v_{h}\left(\beta_{0}, u\right) s_{h}^{(0)}\left(\beta_{0}, u\right) \alpha_{0 h}(u) \mathrm{d} u>\sum_{h=1}^{H-1} \int_{0}^{\tau} l_{k} L \mathrm{~d} u=(H-1) l_{k} .
$$

Então,

$$
\sum_{h=1}^{H-1} \sigma_{k h}^{2} \geq(H-1) l_{k} L \Rightarrow \frac{l_{k}}{\sum_{h=1}^{H-1} \sigma_{k h}^{2}} \leq \frac{1}{(H-1) L}
$$

$\operatorname{Logo}, \frac{l_{k}}{\sum_{h=1}^{H-1} \sigma_{k h}^{2}}=O_{p}(1)$.

\section{Demonstração do Lema A.4}

Usando a expansão de Taylor em $\mathbb{E}_{h}\left(\bar{\beta}^{n}\right)$ em torno de $\beta_{0}(u)$,

$$
\left[\mathbb{E}_{h}(\bar{\beta}, u)-\mathbb{E}_{h}\left(\beta_{0}, u\right)\right]=\left(\bar{\beta}(u)-\beta_{0}(u)\right) \mathbb{V}_{h}\left(\beta_{0}, u\right)+\frac{1}{2}\left(\bar{\beta}(u)-\beta_{0}(u)\right)^{2} \frac{\partial}{\partial \beta(u)} \mathbb{V}_{h}\left(\beta^{*}, u\right),
$$

em que $\left|\beta^{*}(u)-\beta_{0}(u)\right| \leq\left|\beta_{0}(u)-\bar{\beta}(u)\right|$. Então,

$$
\begin{aligned}
& \sup _{0 \leq t \leq \tau}\left|\sqrt{n} \int_{0}^{t}\left[\sum_{k=1}^{K} \mathbb{I}_{k}(u) \frac{l_{k}}{\sum_{h=1}^{H-1} \sigma_{k h}^{2}}\right] \sum_{h=1}^{H-1}\left[\mathbb{E}_{h}\left(\beta_{0}, u\right)-\mathbb{E}_{h}\left(\bar{\beta}^{n}, u\right)\right] S_{h}^{(0)}\left(\beta_{0}, u\right) \alpha_{0 h}(u) \mathrm{d} u\right| \\
\leq & \sum_{h=1}^{H-1} \sup _{0 \leq t \leq \tau}\left|\sqrt{n} \int_{0}^{t}\left[\sum_{k=1}^{K} \mathbb{I}_{k}(u) \frac{l_{k}}{\sum_{h=1}^{H-1} \sigma_{k h}^{2}}\right]\left[\mathbb{E}_{h}\left(\beta_{0}, u\right)-\mathbb{E}_{h}\left(\bar{\beta}^{n}, u\right)\right] S_{h}^{(0)}\left(\beta_{0}, u\right) \alpha_{0 h}(u) \mathrm{d} u\right| \\
= & \sum_{h=1}^{H-1} \sup _{0 \leq t \leq \tau} \mid \sqrt{n} \int_{0}^{t}\left[\sum_{k=1}^{K} \mathbb{I}_{k}(u) \frac{l_{k}}{\sum_{h=1}^{H-1} \sigma_{k h}^{2}}\right] \\
& \cdot\left[\left(\bar{\beta}(u)-\beta_{0}(u)\right) \mathbb{V}_{h}\left(\beta_{0}, u\right)+\frac{1}{2}\left(\bar{\beta}(u)-\beta_{0}(u)\right)^{2} \frac{\partial}{\partial \beta(u)} \mathbb{V}\left(\beta^{*}\right)\right] S_{h}^{(0)}\left(\beta_{0}, u\right) \alpha_{0 h}(u) \mathrm{d} u \mid
\end{aligned}
$$




$$
\begin{aligned}
\leq & \sum_{h=1}^{H-1} \sup _{0 \leq t \leq \tau} \mid \sqrt{n} \int_{0}^{t}\left[\sum_{k=1}^{K} \mathbb{I}_{k}(u) \frac{l_{k}}{\sum_{h=1}^{H-1} \sigma_{k h}^{2}}\right] \\
& .\left(\bar{\beta}(u)-\beta_{0}(u)\right) \mathbb{V}_{h}\left(\beta_{0}, u\right) S_{h}^{(0)}\left(\beta_{0}, u\right) \alpha_{0 h}(u) \mathrm{d} u \mid \\
& +\sum_{h=1}^{H-1} \sup _{0 \leq t \leq \tau} \mid \sqrt{n} \int_{0}^{t}\left[\sum_{k=1}^{K} \mathbb{I}_{k}(u) \frac{l_{k}}{\sum_{h=1}^{H-1} \sigma_{k h}^{2}}\right] \\
& \cdot\left[\frac{1}{2}\left(\bar{\beta}(u)-\beta_{0}(u)\right)^{2} \frac{\partial}{\partial \beta(u)} \mathbb{V}_{h}\left(\beta^{*}\right)\right] S_{h}^{(0)}\left(\beta_{0}, u\right) \alpha_{0 h}(u) \mathrm{d} u \mid .
\end{aligned}
$$

Vamos mostrar que cada uma das parcelas de A.17) é $O_{p}\left(\sqrt{n}\|l\|^{4}\right)$.

$$
\begin{aligned}
& \sup _{0 \leq t \leq \tau}\left|\sqrt{n} \int_{0}^{t}\left[\sum_{k=1}^{K} \mathbb{I}_{k}(u) \frac{l_{k}}{\sum_{h=1}^{H-1} \sigma_{k h}^{2}}\right]\left[\frac{1}{2}\left(\bar{\beta}(u)-\beta_{0}(u)\right)^{2} \frac{\partial}{\partial \beta(u)} \mathbb{V}_{h}\left(\beta^{*}\right)\right] S_{h}^{(0)}\left(\beta_{0}, u\right) \alpha_{0 h}(u) \mathrm{d} u\right| \\
= & \sup _{0 \leq t \leq \tau} \mid \sqrt{n} \int_{0}^{t}\left[\sum_{k=1}^{K} \mathbb{I}_{k}(u) \frac{l_{k}}{\sum_{h=1}^{H-1} \sigma_{k h}^{2}}\right] \frac{1}{2}\left(\bar{\beta}(u)-\beta_{0}(u)\right)^{2} \\
& \left.\cdot \frac{S_{h}^{(3)}\left(\beta^{*}, u\right)}{S_{h}^{(0)}\left(\beta^{*}, u\right)}-3 \frac{S_{h}^{(2)}\left(\beta^{*}, u\right)}{S_{h}^{(0)}\left(\beta^{*}, u\right)} \frac{S_{h}^{(1)}\left(\beta^{*}, u\right)}{S_{h}^{(0)}\left(\beta^{*}, u\right)}+2\left(\frac{S_{h}^{(1)}\left(\beta^{*}, u\right)}{S_{h}^{(0)}\left(\beta^{*}, u\right)}\right)^{2}\right] S_{h}^{(0)}\left(\beta_{0}, u\right) \alpha_{0 h}(u) \mathrm{d} u \mid \\
= & \sup _{0 \leq t \leq \tau}\left|\sqrt{n} \int_{0}^{t}\left[\sum_{k=1}^{K} \mathbb{I}_{k}(u) \frac{l_{k}}{\sum_{h=1}^{H-1} \sigma_{k h}^{2}}\right] \frac{1}{2}\left(\bar{\beta}(u)-\beta_{0}(u)\right)^{2} O_{p}(1) S_{h}^{(0)}\left(\beta_{0}, u\right) \alpha_{0 h}(u) \mathrm{d} u\right| \\
= & \sup _{0 \leq t \leq \tau} \mid \sqrt{n} \int_{0}^{t}\left[\sum_{k=1}^{K} \mathbb{I}_{k}(u) \frac{l_{k}}{\sum_{h=1}^{H-1} \sigma_{k h}^{2}}\right] \frac{1}{2}\left(\bar{\beta}(u)-\beta_{0}(u)\right)^{2} O_{p}(1) S_{h}^{(0)}\left(\beta_{0}, u\right) \mathrm{d} u \\
& -\sqrt{n} \int_{0}^{t}\left[\sum_{k=1}^{K} \mathbb{I}_{k}(u) \frac{l_{k}}{\sum_{h=1}^{H-1} \sigma_{k h}^{2}}\right] \frac{1}{2}\left(\bar{\beta}(u)-\beta_{0}(u)\right)^{2} s_{h}^{(0)}\left(\beta_{0}, u\right) \mathrm{d} u \\
& +\sqrt{n} \int_{0}^{t}\left[\sum_{k=1}^{K} \mathbb{I}_{k}(u) \frac{l_{k}}{\sum_{h=1}^{H-1} \sigma_{k h}^{2}}\right] \frac{1}{2}\left(\bar{\beta}(u)-\beta_{0}(u)\right)^{2} s_{h}^{(0)}\left(\beta_{0}, u\right) \mathrm{d} u \mid
\end{aligned}
$$




$$
\begin{aligned}
\leq & O_{p}(1) \sup _{0 \leq t \leq \tau} \mid \sqrt{n} \int_{0}^{t}\left[\sum_{k=1}^{K} \mathbb{I}_{k}(u) \frac{l_{k}}{\sum_{h=1}^{H-1} \sigma_{k h}^{2}}\right] \frac{1}{2}\left(\bar{\beta}(u)-\beta_{0}(u)\right)^{2}\left[S_{h}^{(0)}\left(\beta_{0}, u\right)-s_{h}^{(0)}\left(\beta_{0}, u\right)\right] \mathrm{d} u \\
& +\sup _{0 \leq t \leq \tau}\left|\sqrt{n} \int_{0}^{t}\left[\sum_{k=1}^{K} \mathbb{I}_{k}(u) \frac{l_{k}}{\sum_{h=1}^{H-1} \sigma_{k h}^{2}}\right] \frac{1}{2}\left(\bar{\beta}(u)-\beta_{0}(u)\right)^{2} s_{h}^{(0)}\left(\beta_{0}, u\right) \mathrm{d} u\right| \\
\leq & O_{p}(1) \sup _{0 \leq t \leq \tau}\left[\sqrt{n} \int_{0}^{t}\left(\bar{\beta}(u)-\beta_{0}(u)\right)^{2}\left|S_{h}^{(0)}\left(\beta_{0}, u\right)-s_{h}^{(0)}\left(\beta_{0}, u\right)\right| \mathrm{d} u\right] \\
& +O(1) \sup _{0 \leq t \leq \tau}\left|\sqrt{n} \int_{0}^{t}\left(\bar{\beta}(u)-\beta_{0}(u)\right)^{2} s_{h}^{(0)}\left(\beta_{0}, u\right) \mathrm{d} u\right| \\
\leq & O_{p}(1)\left[\sqrt{n} \int_{0}^{\tau}\left(\bar{\beta}(u)-\beta_{0}(u)\right)^{2}\left|S_{h}^{(0)}\left(\beta_{0}, u\right)-s_{h}^{(0)}\left(\beta_{0}, u\right)\right|\right] \mathrm{d} u \\
& +O(1)\left|\sqrt{n} \int_{0}^{\tau}\left(\bar{\beta}(u)-\beta_{0}(u)\right)^{2} s_{h}^{(0)}\left(\beta_{0}, u\right)\right| \mathrm{d} u \\
= & O_{p}(1) \sqrt{n}\|l\|^{4}=O_{p}\left(\sqrt{n}\|l\|^{4}\right) .
\end{aligned}
$$

Com relação ao termo A.16,

$$
\begin{aligned}
& \sup _{0 \leq t \leq \tau}\left|\sqrt{n} \int_{0}^{t}\left[\sum_{k=1}^{K} \mathbb{I}_{k}(u) \frac{l_{k}}{\sum_{h=1}^{H-1} \sigma_{k h}^{2}}\right]\left[\bar{\beta}(u)-\beta_{0}(u)\right] \mathbb{V}_{h}\left(\beta_{0}, u\right) S_{h}^{(0)}\left(\beta_{0}, u\right) \alpha_{0 h}(u) \mathrm{d} u\right| \\
= & \sup _{0 \leq t \leq \tau} \mid \sqrt{n} \int_{0}^{t}\left[\sum_{k=1}^{K} \mathbb{I}_{k}(u) \frac{l_{k}}{\sum_{h=1}^{H-1} \sigma_{k h}^{2}}\right]\left[\bar{\beta}(u)-\beta_{0}(u)\right] \mathbb{V}_{h}\left(\beta_{0}, u\right) S_{h}^{(0)}\left(\beta_{0}, u\right) \alpha_{0 h}(u) \mathrm{d} u \\
& -\sqrt{n} \int_{0}^{t}\left[\bar{\beta}(u)-\beta_{0}(u)\right] \mathrm{d} u+\sqrt{n} \int_{0}^{t}\left[\bar{\beta}(u)-\beta_{0}(u)\right] \mathrm{d} u \mid \\
\leq & \sup _{0 \leq t \leq \tau}\left|\sqrt{n} \int_{0}^{t}\left[\bar{\beta}(u)-\beta_{0}(u)\right] \mathrm{d} u\right| \\
& +\sup _{0 \leq t \leq \tau}\left|\sqrt{n} \int_{0}^{t}\right| \bar{\beta}(u)-\beta_{0}(u) \mid\left[\sum_{k=1}^{K} \mathbb{I}_{k}(u) \frac{l_{k}}{\sum_{h=1}^{H-1} \sigma_{k h}^{2}}\right] \\
& . \mathbb{V}_{h}\left(\beta_{0}, u\right) S_{h}^{(0)}\left(\beta_{0}, u\right) \alpha_{0 h}(u)-1 \mathrm{~d} u .
\end{aligned}
$$


O termo A.18, é $O\left(\sqrt{n}\|l\|^{4}\right)$ conforme já mostrado em Murphy \& Sen (1991). Além disso, $\left[\frac{l_{k}}{\sum_{h=1}^{H-1} \sigma_{k h}^{2}}\right]=O(1)$. Quanto ao termo A.19, podemos reescrevê-lo como

$$
\begin{aligned}
& \sup _{0 \leq t \leq \tau}\left|\sqrt{n} \int_{0}^{t}\right|\left(\bar{\beta}(u)-\beta_{0}(u)\right)\left|\left[\sum_{k=1}^{K} \mathbb{I}_{k}(u) \frac{l_{k}}{\sum_{h=1}^{H-1} \sigma_{k h}^{2}}\right] \mathbb{V}_{h}\left(\beta_{0}, u\right) S_{h}^{(0)}\left(\beta_{0}, u\right) \alpha_{0 h}(u)-1\right| \mathrm{d} u \\
= & \sup _{0 \leq t \leq \tau}\left|\sqrt{n} \int_{0}^{t}\right|\left(\bar{\beta}(u)-\beta_{0}(u)\right)\left|O(1)\left\{\mathbb{V}_{h}\left(\beta_{0}, u\right) S_{h}^{(0)}\left(\beta_{0}, u\right) \alpha_{0 h}(u)-\left[\sum_{k=1}^{K} \mathbb{I}_{k}(u) \frac{l_{k}}{\sum_{h=1}^{H-1} \sigma_{k h}^{2}}\right]\right\}\right| \mathrm{d} u \\
\leq & O(1) \sup _{0 \leq t \leq \tau}\left|\sqrt{n} \int_{0}^{t}\right|\left(\bar{\beta}(u)-\beta_{0}(u)\right) \mid\left\{\mathbb{V}_{h}\left(\beta_{0}, u\right) S_{h}^{(0)}\left(\beta_{0}, u\right) \alpha_{0 h}(u)\right. \\
& \left.-v_{h}\left(\beta_{0}, u\right) s_{h}^{(0)}\left(\beta_{0}, u\right) \alpha_{0 h}(u)+v_{h}\left(\beta_{0}, u\right) s_{h}^{(0)}\left(\beta_{0}, u\right) \alpha_{0 h}(u)-\left[\sum_{k=1}^{K} \mathbb{I}_{k}(u) \frac{\sum_{h=1}^{H-1} \sigma_{k h}^{2}}{l_{k}}\right]\right\} \mid \mathrm{d} u \\
\leq & O(1) \sup _{0 \leq t \leq \tau}\left|\sqrt{n} \int_{0}^{t}\right|\left(\bar{\beta}(u)-\beta_{0}(u)\right) \mid \\
& \cdot\left\{\mathbb{V}_{h}\left(\beta_{0}, u\right) S_{h}^{(0)}\left(\beta_{0}, u\right) \alpha_{0 h}(u)-v_{h}\left(\beta_{0}, u\right) s_{h}^{(0)}\left(\beta_{0}, u\right) \alpha_{0 h}(u)\right\} \mid \mathrm{d} u \\
& +\sup _{0 \leq t \leq \tau}\left|\sqrt{n} \int_{0}^{t}\right|\left(\bar{\beta}(u)-\beta_{0}(u)\right) \mid O(1) \\
& \left.\quad\left\{v_{h}\left(\beta_{0}, u\right) s_{h}^{(0)}\left(\beta_{0}, u\right) \alpha_{0 h}(u)-\left[\sum_{k=1}^{K} \mathbb{I}_{k}(u) \frac{l_{k}}{\sum_{h=1}^{H-1} \sigma_{k h}^{2}}\right]\right\} \mid \mathrm{d} u .20\right)
\end{aligned}
$$


O termo A.20 é

$$
\begin{aligned}
& \sqrt{n} \int_{0}^{\tau}\left|\bar{\beta}(u)-\beta_{0}(u)\right|\left|\mathbb{V}_{h}\left(\beta_{0}, u\right) S_{h}^{(0)}\left(\beta_{0}, u\right)-v_{h}\left(\beta_{0}, u\right) s_{h}^{(0)}\left(\beta_{0}, u\right)\right| \alpha_{0 h}(u) \mathrm{d} u \\
\leq & \sqrt{n} \sup _{0 \leq u \leq \tau}\left|\bar{\beta}(u)-\beta_{0}(u)\right| \int_{0}^{\tau}\left|\mathbb{V}_{h}\left(\beta_{0}, u\right) S_{h}^{(0)}\left(\beta_{0}, u\right)-v_{h}\left(\beta_{0}, u\right) s_{h}^{(0)}\left(\beta_{0}, u\right)\right| \alpha_{0 h}(u) \mathrm{d} u \\
\leq & \sqrt{n} \sup _{0 \leq u \leq \tau}\left|\bar{\beta}(u)-\beta_{0}(u)\right| O\left(\frac{1}{\sqrt{n}}\right) \\
= & O(1) \int_{0}^{\tau}\left|\bar{\beta}(u)-\beta_{0}(u)\right| \mathrm{d} u \\
= & O(1)\|l\|^{2}=O\left(\|l\|^{2}\right) .
\end{aligned}
$$

Agora, considere $f(u)=v_{h}\left(\beta_{0}, u\right) s_{h}^{(0)}\left(\beta_{0}, u\right) \alpha_{0 h}(u)$. Então,

$$
\sigma_{k}^{2}=\int_{0}^{\tau} \mathbb{I}_{k}(u) v_{h}\left(\beta_{0}, u\right) s_{h}^{(0)}\left(\beta_{0}, u\right) \alpha_{0 h}(u)=\int_{0}^{\tau} \mathbb{I}_{k}(u) f(u) \mathrm{d} u
$$

Assim, o termo A.21 pode ser escrito como

$$
\begin{aligned}
& \sqrt{n} \int_{0}^{\tau}\left[\bar{\beta}(u)-\beta_{0}(u)\right]\left[f(u)-\sum_{k=1}^{K} \mathbb{I}_{k}(u) \frac{\sigma_{k}^{2}}{l_{k}}\right] \mathrm{d} u \\
= & \sqrt{n} \int_{0}^{\tau}\left[\bar{\beta}(u)-\beta_{0}(u)\right]\left[\sum_{k=1}^{K} \mathbb{I}_{k}(u) \frac{l_{k}}{l_{k}} f(u)-\sum_{k=1}^{K} \mathbb{I}_{k}(u) \frac{\sigma_{k}^{2}}{l_{k}}\right] \mathrm{d} u \\
= & \sqrt{n} \int_{0}^{\tau}\left[\bar{\beta}(u)-\beta_{0}(u)\right]\left[\sum_{k=1}^{K} \mathbb{I}_{k}(u) \frac{1}{l_{k}}\left(l_{k} f(u)-\sigma_{k}^{2}\right)\right] \mathrm{d} u \\
= & \sqrt{n} \int_{0}^{\tau}\left[\bar{\beta}(u)-\beta_{0}(u)\right]\left[\sum_{k=1}^{K} \mathbb{I}_{k}(u) \frac{1}{l_{k}}\left(\int_{0}^{\tau} \mathbb{I}_{k}(s) f(u) \mathrm{d} s-\int_{0}^{\tau} \mathbb{I}_{k}(s) f(s) \mathrm{d} s\right)\right] \mathrm{d} u
\end{aligned}
$$


Da condição de Lipshitz

$$
\begin{aligned}
& =\sqrt{n} \int_{0}^{\tau}\left[\bar{\beta}(u)-\beta_{0}(u)\right]\left[\sum_{k=1}^{K} \mathbb{I}_{k}(u) \frac{1}{l_{k}}\left(\int_{0}^{\tau} \mathbb{I}_{k}(s)|f(u)-f(s)| \mathrm{d} s\right)\right] \mathrm{d} u \\
& \leq c \sqrt{n} \int_{0}^{\tau}\left[\bar{\beta}(u)-\beta_{0}(u)\right]\left[\sum_{k=1}^{K} \mathbb{I}_{k}(u) \frac{1}{l_{k}}\left(\int_{0}^{\tau} \mathbb{I}_{k}(s)|u-s| \mathrm{d} s\right)\right] \mathrm{d} u \\
& \leq c \sqrt{n} \int_{0}^{\tau}\left[\bar{\beta}(u)-\beta_{0}(u)\right]\left[\sum_{k=1}^{K} \mathbb{I}_{k}(u) \frac{1}{l_{k}}\left(\int_{0}^{\tau} \mathbb{I}_{k}(s) l_{k} \mathrm{~d} s\right)\right] \mathrm{d} u \\
& \leq O_{p}(1) \sqrt{n} \int_{0}^{\tau}\left[\bar{\beta}(u)-\beta_{0}(u)\right]\left[\sum_{k=1}^{K} \mathbb{I}_{k}(u) l_{k}\right] \mathrm{d} u \\
& =O(1) l_{(K)} \sqrt{n} \int_{0}^{\tau}\left[\bar{\beta}(u)-\beta_{0}(u)\right]\left[\sum_{k=1}^{K} \mathbb{I}_{k}(u)\right] \mathrm{d} u \\
& =O\left(\|l\|^{2}\right) \sqrt{n} \int_{0}^{\tau}\left[\bar{\beta}(u)-\beta_{0}(u)\right]\left[\sum_{k=1}^{K} \mathbb{I}_{k}(u)\right] \mathrm{d} u \\
& =O\left(\|l\|^{2}\right) \sqrt{n}\|l\|^{2}=O\left(\sqrt{n}\|l\|^{4}\right), \quad \operatorname{por} \mathrm{D}(3) .
\end{aligned}
$$




\section{Apêndice B}

\section{Resultados}

\section{B.1 Cálculo de $\bar{\beta}^{n}$}

Expandindo $\mathcal{L}_{n}\left(\beta^{n}\right)$ em torno de $\beta_{0}$,

$$
\begin{aligned}
\mathcal{L}_{n}\left(\beta^{n}\right)= & \sum_{h=1}^{H-1} \mathcal{L}_{n h}\left(\beta^{n}\right)=\sum_{h=1}^{H-1}\left[\mathcal{L}_{n h}\left(\beta_{0}^{n}\right)+\frac{\partial \mathcal{L}_{n h}\left(\beta_{0}^{n}\right)}{\partial \beta}\left[\beta(u)-\beta_{0}(u)\right]\right. \\
& \left.+\frac{1}{2} \frac{\partial^{2} \mathcal{L}_{n h}\left(\beta_{0}^{n}\right)}{\partial \beta^{2}}\left[\beta(u)-\beta_{0}(u)\right]^{2}\right] .
\end{aligned}
$$

Então,

$$
\begin{aligned}
& \sum_{h=1}^{H-1}\left[\mathcal{L}_{n h}\left(\beta^{n}\right)-\mathcal{L}_{n h}\left(\beta_{0}^{n}\right)\right]=\sum_{h=1}^{H-1}\left[\frac{\partial \mathcal{L}_{n h}\left(\beta_{0}^{n}\right)}{\partial \beta}\left[\beta(u)-\beta_{0}(u)\right]+\frac{1}{2} \frac{\partial^{2} \mathcal{L}_{n h}\left(\beta_{0}^{n}\right)}{\partial \beta^{2}}\left[\beta(u)-\beta_{0}(u)\right]^{2}\right] \\
& \sum_{h=1}^{H-1}\left[\mathcal{L}_{n h}\left(\beta^{n}\right)-\mathcal{L}_{n h}\left(\beta_{0}^{n}\right)\right]=\sum_{h=1}^{H-1}\left[\sum_{i=1}^{n} \int_{0}^{\tau}\left[X_{i}-\frac{S_{h}^{(1)}\left(\beta_{0}, u\right)}{S_{h}^{(0)}\left(\beta_{0}, u\right)}\right]\left[\beta(u)-\beta_{0}(u)\right]\right] \mathrm{d} N_{h i}(u) \quad(\mathrm{B} .1) \\
& +\sum_{h=1}^{H-1} \frac{1}{2}\left[\sum_{i=1}^{n} \int_{0}^{\tau}\left[\frac{S_{h}^{(2)}\left(\beta_{0}, u\right)}{S_{h}^{(0)}\left(\beta_{0}, u\right)}-\left(\frac{S_{h}^{(1)}\left(\beta_{0}, u\right)}{S_{h}^{(0)}\left(\beta_{0}, u\right)}\right)\right]^{2} \cdot\left[\beta(u)-\beta_{0}(u)\right]^{2}\right] \mathrm{d} N_{h i}(u) .
\end{aligned}
$$


Usando o fato que $\mathrm{d} N_{h i}(u)=\mathrm{d} \Lambda_{h i}(u)+\mathrm{d} M_{h i}(u)$, podemos reescrever o primeiro termo (B.1) como

$$
\begin{aligned}
& \sum_{h=1}^{H-1}\left[\sum_{i=1}^{n} \int_{0}^{\tau}\left[X_{i}-\frac{S_{h}^{(1)}\left(\beta_{0}, u\right)}{S_{h}^{(0)}\left(\beta_{0}, u\right)}\right]\left[\beta(u)-\beta_{0}(u)\right] \mathrm{d} N_{h i}(u)\right] \\
= & \sum_{h=1}^{H-1}\left[\sum_{i=1}^{n} \int_{0}^{\tau}\left[X_{i}-\frac{S_{h}^{(1)}\left(\beta_{0}, u\right)}{S_{h}^{(0)}\left(\beta_{0}, u\right)}\right]\left[\beta(u)-\beta_{0}(u)\right] \mathrm{d} M_{h i}(u)\right] \\
& +\sum_{h=1}^{H-1}\left[\sum_{i=1}^{n} \int_{0}^{\tau}\left[X_{i}-\frac{S_{h}^{(1)}\left(\beta_{0}, u\right)}{S_{h}^{(0)}\left(\beta_{0}, u\right)}\right]\left[\beta(u)-\beta_{0}(u)\right] \mathrm{d} \Lambda_{h i}(u)\right] .
\end{aligned}
$$

O primeiro termo (B.3) representa somas de martingais de média zero e o segundo termo B.4 também é zero, pois

$$
\begin{aligned}
& \frac{1}{n} \sum_{i=1}^{n} \sum_{h=1}^{H-1} \int_{0}^{\tau} \frac{\partial \mathcal{L}_{n h}\left(\beta_{0}^{n}\right)}{\partial \beta}\left[\beta(u)-\beta_{0}(u)\right] \mathrm{d} \Lambda_{h i}(u) \\
= & \frac{1}{n} \sum_{i=1}^{n} \sum_{h=1}^{H-1} \int_{0}^{\tau}\left[X_{i}-\frac{S_{h}^{(1)}\left(\beta_{0}, u\right)}{S_{h}^{(0)}\left(\beta_{0}, u\right)}\right]\left[\beta(u)-\beta_{0}(u)\right] e^{X_{i} \beta_{0}(u)} Y_{i h}(u) \mathrm{d} u \\
= & \frac{1}{n} \sum_{h=1}^{H-1} \int_{0}^{\tau}\left[\sum_{i=1}^{n} X_{i} e^{X_{i} \beta_{0}(u)} Y_{i h}(u)-\frac{S_{h}^{(1)}\left(\beta_{0}, u\right)}{S_{h}^{(0)}\left(\beta_{0}, u\right)} \sum_{i=1}^{n} e^{X_{i} \beta_{0}(u)} Y_{i h}(u)\right]\left[\beta(u)-\beta_{0}(u)\right] \alpha_{0 h}(u) \mathrm{d} u \\
= & \frac{1}{n} \sum_{h=1}^{H-1} \int_{0}^{\tau}\left[n S_{h}^{(1)}\left(\beta_{0}, u\right)-\frac{S^{(1)}\left(\beta_{0}, u\right)}{S^{(0)}\left(\beta_{0}, u\right)} n S^{(0)}\left(\beta_{0}, u\right)\right]\left[\beta(u)-\beta_{0}(u)\right] \alpha_{0 h}(u) \mathrm{d} u \\
= & \frac{1}{n} \sum_{h=1}^{H-1} \int_{0}^{\tau}\left[n S^{(1)}\left(\beta_{0}, u\right)-S_{h}^{(1)}\left(\beta_{0}, u\right) n\right]\left[\beta(u)-\beta_{0}(u)\right] \alpha_{0 h}(u) \mathrm{d} u=0
\end{aligned}
$$

Logo, o primeiro termo (B.1) é desprezível. Considerando agora, o segundo termo (B.2) e usando novamente o fato $\mathrm{d} N_{h i}(u)=\mathrm{d} \Lambda_{h i}(u)+\mathrm{d} M_{h i}(u)$, temos,

$$
\frac{1}{2 n} \sum_{i=1}^{n} \sum_{h=1}^{H-1} \int_{0}^{\tau} \mathbb{V}_{h}\left(\beta_{0}, u\right)\left(\beta(u)-\beta_{0}(u)\right)^{2} \mathrm{~d} N_{h i}(u)
$$




$$
\begin{aligned}
= & \frac{1}{2 n} \sum_{i=1}^{n} \sum_{h=1}^{H-1} \int_{0}^{\tau} \mathbb{V}_{h}\left(\beta_{0}, u\right)\left[\beta(u)-\beta_{0}(u)\right]^{2} \mathrm{~d} M_{h i}(u) \\
& +\frac{1}{2 n} \sum_{i=1}^{n} \sum_{h=1}^{H-1} \int_{0}^{\tau} \mathbb{V}_{h}\left(\beta_{0}, u\right)\left[\beta(u)-\beta_{0}(u)\right]^{2} \mathrm{~d} \Lambda_{h i}(u) .
\end{aligned}
$$

O primeiro termo é uma soma de martingais de média zero. Então maximizar

$$
\frac{1}{2} \sum_{i=1}^{n} \sum_{h=1}^{H-1} \int_{0}^{\tau} \mathbb{V}_{h}\left(\beta_{0}, u\right) S_{h}^{(0)}\left(\beta_{0}, u\right) \alpha_{0 h}(u)\left[\beta(u)-\beta_{0}(u)\right]^{2} \mathrm{~d} u
$$

em $\beta$, equivale, assintoticamente, a resolver a equação

$$
\sum_{h=1}^{H-1} \int_{0}^{\tau} v_{h}\left(\beta_{0}, u\right) s_{h}^{(0)}\left(\beta_{0}, u\right) \alpha_{0 h}(u)\left[\beta(u)-\beta_{0}(u)\right] \mathrm{d} u=0
$$

$\log \mathrm{O}$

$$
\sum_{h=1}^{H-1} \int_{0}^{\tau} v_{h}\left(\beta_{0}, u\right) s_{h}^{(0)}\left(\beta_{0}, u\right) \alpha_{0 h}(u) \beta(u) \mathrm{d} u=\sum_{h=1}^{H-1} \int_{0}^{\tau} v_{h}\left(\beta_{0}, u\right) s_{h}^{(0)}\left(\beta_{0}, u\right) \alpha_{0 h}(u) \beta_{0}(u) \mathrm{d} u
$$

Considerando que $\beta(u)$ é um crivo,

$$
\sum_{h=1}^{H-1} \int_{0}^{\tau} v_{h}\left(\beta_{0}, u\right) s_{h}^{(0)}\left(\beta_{0}, u\right) \alpha_{0 h}(u) \sum_{k=1}^{K} \bar{\beta}_{k}^{n} \mathbb{I}_{k}(u)=\sum_{h=1}^{H-1} \int_{0}^{\tau} v_{h}\left(\beta_{0}, u\right) s_{h}^{(0)}\left(\beta_{0}, u\right) \alpha_{0 h}(u) \beta_{0}(u) \mathrm{d} u
$$

para um dado intervalo $k$, temos $\bar{\beta}_{k}$ constante, assim

$$
\bar{\beta}_{k} \sum_{h=1}^{H-1} \int_{0}^{\tau} \mathbb{I}_{k}(u) v_{h}\left(\beta_{0}, u\right) s_{h}^{(0)}\left(\beta_{0}, u\right) \alpha_{0 h}(u) \mathrm{d} u=\sum_{h=1}^{H-1} \int_{0}^{\tau} \mathbb{I}_{k}(u) v_{h}\left(\beta_{0}, u\right) s_{h}^{(0)}\left(\beta_{0}, u\right) \alpha_{0 h}(u) \beta_{0}(u) \mathrm{d} u .
$$


De onde concluímos que

$$
\bar{\beta}_{k}=\frac{\sum_{h=1}^{H-1} \int_{0}^{\tau} \mathbb{I}_{k}(u) \beta_{0}(u) v_{h}\left(\beta_{0}, u\right) s_{h}^{(0)}\left(\beta_{0}, u\right) \alpha_{0 h}(u) \mathrm{d} u}{\sum_{h=1}^{H-1} \int_{0}^{\tau} \mathbb{I}_{k}(u) v_{h}\left(\beta_{0}, u\right) s_{h}^{(0)}\left(\beta_{0}, u\right) \alpha_{0 h}(u) \mathrm{d} u} .
$$

Assim, para o intervalo todo temos

$$
\bar{\beta}^{n}(u)=\sum_{k=1}^{K} \bar{\beta}_{k} \mathbb{I}_{k}(u) .
$$

\section{B.2 Cálculo da primeira parte do Teorema 1}

Provar que $\int_{0}^{\tau}\left[\bar{\beta}^{n}(u)-\beta_{0}(u)\right]^{2} \mathrm{~d} u=O_{p}\left(\|l\|^{4}\right)$.

Da mesma forma apresentada em Murphy \& Sen (1991), usamos a definição de $\bar{\beta}^{n}(u)$, condição $\mathrm{D}(1)$ e $\lim \sup \left(\frac{l_{(K)}}{l_{(1)}}\right)<\infty$.

Note que

$$
\begin{aligned}
& \left|\bar{\beta}^{n}(u)-\beta_{0}(u)\right|=\sum_{k=1}^{K}\left[\bar{\beta}_{k}-\beta_{0}(u)\right] \mathbb{I}_{k}(u) \\
= & \sum_{k=1}^{K}\left[\frac{1}{\sigma_{k}^{2}} \sum_{h=1}^{H-1} \int_{0}^{\tau} \mathbb{I}_{k}(s) \beta_{0}(s) v_{h}\left(\beta_{0}, s\right) s_{h}^{(0)}\left(\beta_{0}, s\right) \alpha_{0 h}(s) \mathrm{d} s-\beta_{0}(u)\right] \mathbb{I}_{k}(u) \\
= & \sum_{k=1}^{K}\left\{\frac { 1 } { \sigma _ { k } ^ { 2 } } \left[\sum_{h=1}^{H-1} \int_{0}^{\tau} \mathbb{I}_{k}(s) \beta_{0}(s) v_{h}\left(\beta_{0}, s\right) s_{h}^{(0)}\left(\beta_{0}, s\right) \alpha_{0 h}(s) \mathrm{d} s\right.\right. \\
& \left.\left.-\sum_{h=1}^{H-1} \int_{0}^{\tau} \mathbb{I}_{k}(s) \beta_{0}(u) v_{h}\left(\beta_{0}, s\right) s_{h}^{(0)}\left(\beta_{0}, s\right) \alpha_{0 h}(s) \mathrm{d} s\right]\right\} \mathbb{I}_{k}(u)
\end{aligned}
$$




$$
\begin{aligned}
& \left.=\sum_{k=1}^{K}\left\{\frac{1}{\sigma_{k}^{2}}\left[\sum_{h=1}^{H-1} \int_{0}^{\tau} \mathbb{I}_{k}(s)\left|\beta_{0}(s)-\beta_{0}(u)\right| v_{h}\left(\beta_{0}, s\right) s_{h}^{(0)}\left(\beta_{0}, s\right) \alpha_{0 h}(s) \mathrm{d} s\right]\right\} \mathbb{I}_{k}(u) \quad \text { (condição } \mathrm{D}(1),\right) \\
& \leq c \sum_{k=1}^{K}\left\{\frac{1}{\sigma_{k}^{2}}\left[\sum_{h=1}^{H-1} \int_{0}^{\tau} \mathbb{I}_{k}(s)|s-u| v_{h}\left(\beta_{0}, s\right)^{(0)} S_{h}\left(\beta_{0}, s\right) \alpha_{0 h}(s) \mathrm{d} s\right]\right\} \mathbb{I}_{k}(u) \\
& \leq c \sum_{k=1}^{K}\left\{\frac{1}{\sigma_{k}^{2}} l_{k}\left[\int_{0}^{\tau} \mathbb{I}_{k}(s) v_{h}\left(\beta_{0}, s\right) s_{h}^{(0)}\left(\beta_{0}, s\right) \alpha_{0 h}(s) \mathrm{d} s\right]\right\} \mathbb{I}_{k}(u) \\
& =O(1) \sum_{k=1}^{K} l_{k} \mathbb{I}_{k}(u) .
\end{aligned}
$$

Alguns resultados que decorrem disso são:

(a)

$$
\begin{aligned}
\int_{0}^{\tau}\left(\bar{\beta}^{n}(u)-\beta_{0}(u)\right)^{2} \mathrm{~d} u & =O(1) \int_{0}^{\tau}\left[\sum_{k=1}^{K} l_{k} \mathbb{I}_{k}(u)\right]^{2} \mathrm{~d} u \\
& =O(1) \int_{0}^{\tau} \sum_{k=1}^{K} l_{k}^{2} \mathbb{I}_{k}(u) \mathrm{d} u=O(1) \sum_{k=1}^{K} l_{k}^{2} \int_{0}^{\tau} \mathbb{I}_{k}(u) \mathrm{d} u \\
& =O(1) \sum_{k=1}^{K} l_{k}^{2} l_{k} \leq O(1) l_{(K)} \sum_{k=1}^{K} l_{k}^{2} \\
& =O(1) l_{(K)}\|l\|^{2} \\
& \leq O(1)\|l\|^{2}\|l\|^{2}=O\left(\|l\|^{4}\right)
\end{aligned}
$$

(b)

$$
\begin{aligned}
& \sup _{0 \leq u \leq \tau}\left|\bar{\beta}^{n}(u)-\beta_{0}(u)\right|=\sup _{0 \leq u \leq \tau}\left[O(1) \sum_{k=1}^{K} l_{k} \mathbb{I}_{k}(u)\right] \\
& \leq O(1) l_{(K)} \sup _{0 \leq u \leq \tau} \sum_{k=1}^{K} \mathbb{I}_{k}(u)=O\left(l_{(K)}\right)=O\left(\|l\|^{2}\right) .
\end{aligned}
$$


(c)

$$
\begin{aligned}
& \int_{0}^{\tau}\left(\widehat{\beta}^{n}(u)-\bar{\beta}^{n}(u)\right)^{2} \mathrm{~d} u=\int_{0}^{\tau} \sum_{k=1}^{K}\left(\widehat{\beta}_{k}^{n}-\bar{\beta}_{k}^{n}\right)^{2} \mathbb{I}_{k}(u) \mathrm{d} u \\
= & \sum_{k=1}^{K}\left(\widehat{\beta}_{k}^{n}-\bar{\beta}_{k}^{n}\right)^{2} \int_{0}^{\tau} \mathbb{I}_{k}(u) \mathrm{d} u \leq\|\widehat{\beta}-\bar{\beta}\|^{2} l_{(K)} \leq O\left(\|l\|^{2}\right)\|\widehat{\beta}-\bar{\beta}\|^{2} .
\end{aligned}
$$

\section{B.3 Resultados úteis}

(1)

$$
\begin{aligned}
& \int_{0}^{\tau} \mathbb{I}_{k}(u)\left(\bar{\beta}^{n}(u)-\beta_{0}(u)\right) v_{h}\left(\beta_{0}, u\right) s_{h}^{(0)}\left(\beta_{0}, u\right) \alpha_{0 h}(u) \mathrm{d} u \\
= & \int_{0}^{\tau} \mathbb{I}_{k}(u) \bar{\beta}^{n}(u) v_{h}\left(\beta_{0}, u\right) s_{h}^{(0)}\left(\beta_{0}, u\right) \alpha_{0 h}(u) \mathrm{d} u-\int_{0}^{\tau} \mathbb{I}_{k}(u) \beta_{0}(u) v_{h}\left(\beta_{0}, u\right) s_{h}^{(0)}\left(\beta_{0}, u\right) \alpha_{0 h}(u) \mathrm{d} u \\
& \left(\text { por definição de } \bar{\beta}_{k}\right) \\
= & \int_{0}^{\tau} \mathbb{I}_{k}(u) \bar{\beta}^{n}(u) v_{h}\left(\beta_{0}, u\right) s_{h}^{(0)}\left(\beta_{0}, u\right) \alpha_{0 h}(u) \mathrm{d} u-\bar{\beta}_{k} \sigma_{k}^{2} \\
& \left(\bar{\beta}_{k} \text { é constante no intervalo } I_{k}\right) \\
= & \bar{\beta}_{k} \int_{0}^{\tau} \mathbb{I}_{k}(u) v_{h}\left(\beta_{0}, u\right) s_{h}^{(0)}\left(\beta_{0}, u\right) \alpha_{0 h}(u) \mathrm{d} u-\bar{\beta}_{k} \sigma_{k}^{2} \\
= & \bar{\beta}_{k} \sigma_{k}^{2}-\bar{\beta}_{k} \sigma_{k}^{2}=0 .
\end{aligned}
$$

$$
\begin{aligned}
& \int_{0}^{\tau} \sum_{k=1}^{K} \mathbb{I}_{k}(u) l_{k}^{-2} v_{h}\left(\beta_{0}, u\right) s_{h}^{(0)}\left(\beta_{0}, u\right) \alpha_{0 h}(u) \mathrm{d} u \\
= & O_{p}(1) \int_{0}^{\tau} \sum_{k=1}^{K} \mathbb{I}_{k}(u) l_{k}^{-2} \mathrm{~d} u \leq O_{p}(1) l_{(1)}^{-2} \sum_{k=1}^{K} \int_{0}^{\tau} \mathbb{I}_{k}(u) \mathrm{d} u \\
\leq & O_{p}(1)\|l\|^{-4} \sum_{k=1}^{K} l_{k}=O_{p}\left(\|l\|^{-4}\right) .
\end{aligned}
$$


(3)

$$
\begin{aligned}
& \frac{\|l\|^{2}}{l_{(1)}}=\frac{\sum_{k=1}^{K} l_{k}^{2}}{l_{(1)}}=\frac{l_{(K)}}{l_{(1)}} \sum_{k=1}^{K} l_{k}=\frac{l_{(K)}}{l_{(1)}} \tau<\infty \\
& \tau<\infty \log , \\
& \frac{\|l\|^{2}}{l_{(1)}}<\infty \quad \Rightarrow \frac{\|l\|^{2}}{l_{(1)}} \leq M_{1}
\end{aligned}
$$

(4)

$$
\begin{aligned}
& \frac{l_{(K)}}{\|l\|^{2}}=\frac{l_{(K)}}{\sum_{k=1}^{K} l_{k}^{2}}=\frac{l_{(K)}}{l_{(1)} \sum_{k=1}^{K} l_{k}}=\frac{l_{(K)}}{l_{(1)} \tau}<\infty \\
& \tau<\infty \log 0, \\
& \frac{l_{(K)}}{\|l\|^{2}}<\infty \quad \Rightarrow \frac{l_{(K)}}{\|l\|^{2}} \leq M_{2}
\end{aligned}
$$




\section{Referências}

Aalen, O. \& Johansen, S. (1978). An empirical transition matrix for nonhomogeneous Markov chains based on censored observations, Scandinavian Journal of Statistics 5: 141-150.

Aalen, O. O. (1989). A linear regression model for the analysis of life times, Statistics in Medicine 8: 907-925.

Aalen, O. O., Borgan, Ø. \& Fekjaer, H. (2001). Covariate adjustment of event histories estimated from Markov chains: the additive approach, Biometrics 57: 993-1001.

Aitchison, J. \& Silvey, S. D. (1958). Maximum-likelihood estimation of parameters subject to restraints, Annals of Mathematical Statistics 29: 813-828.

Andersen, P. K., Abildstrom, S. Z. \& Rosthøj, S. (2002). Competing risks as a multi-state model, Statistical Methods in Medical Research 11: 203-215.

Andersen, P. K., Borgan, Ø., Gill, R. D. \& Keiding, N. (1993). Statistical models based on counting processes, New York: Springer-Verlag.

Andersen, P. K. \& Gill, R. D. (1982). Cox's regression model for counting processes: A large sample study, The Annals of Statistics 10: 1100-1120.

Chiang, C. (1980). An Introduction to stochastic processes and their applications, Huntington, New York: R. E. Krieger Pub. Co.

Commenges, D. (1999). Multi-state models in epidemiology, Lifetime Data Analysis 5: 315327. 
Cox, D. \& Oakes, D. (1984). Analysis of Survival Data, London: Chapman and Hall.

Fleming, T. R. (1978). Nonparametric estimation for nonhomogeneous Markov processes in the problem of competing risks, The Annals of Statistics 6: 1057-1070.

Frydman, H. (1992). A nonparametric estimation procedure for a periodically observed threestate Markov process, with application to AIDS, Journal of the Royal Statistical Society, Series B 54: 853-866.

Frydman, H. (1995). Semiparametric estimation in a three-state duration-dependent Markov model from interval-censored observations with application to AIDS data, Biometrics 51: 502-511.

Gill, R. \& Johansen, S. (1990). A survey of product-integration with a view towards application in survival analysis, Annals of Statistics 18: 1501-1555.

Grenander, U. (1981). Abstract inference, New York: Wiley.

Grüger, J., Kay, R. \& Schumacher, M. (1991). The validity of inferences based on incomplete observations in disease state models, Biometrics 47: 595-605.

Hastie, T. \& Tibshirani, R. (1993). Varying-coefficient models (with discussion), Journal of the Royal Statistical Society, Series B 55: 757-796.

Hogan, J. W. \& Laird, N. M. (1997). Model-based approaches to analysing incomplete longitudinal and failure time data, Statistics in Medicine 16: 259-272.

Hougaard, P. (2000). Analysis of multivariate survival data, New York: Springer.

Hsieh, H.-J., Chen, T. H.-H. \& Chang, S.-H. (2002). Assessing chronic disease progression using non-homogeneous exponential regression Markov models: an illustration using a selective breast cancer screening in Taiwan, Statistics in Medicine 21: 3369-3382.

Jackson, C. (2007). The msm package, http://cran.fiocruz.br/src/contrib/Descriptions/msm.html. 
Joly, P. \& Commenges, D. (1999). A penalized likelihood approach for a progressive three-state model with censored and truncated data: application to AIDS, Biometrics 55: 887-890.

Joly, P., Commenges, D., Helmer, C. \& Letenneur, L. (2002). A penalized likelihood approach for an illness-death model with interval-censored data: application to age-specific incidence of dementia, Biostatistics 3: 433-443.

Kaplan, E. \& Meier, P. (1958). Non-parametric estimation from incomplete observations, Journal of the America Statistical Association 53: 457-481,562-563.

Kay, R. (1986). A Markov model for analysing cancer markers and disease states in survival studies, Biometrics 42: 855-865.

Keiding, N. (1991). Age-specific incidence and prevalence: a statistical perspective, Journal of the Royal Statistical Society, Series A 154: 371-412.

Lagakos, S. W., Sommer, C. J. \& Zelen, M. (1978). Semi-Markov models for partially censored data, Biometrika 65: 311-317.

Marshall, G. \& Jones, R. (1995). Multi-state models and diabetic retinopathy, Statistics in Medicine 14: 1975-1983.

Martinussen, T., Scheike, T. H. \& Skovgaard, I. M. (2002). Efficient estimation of fixed and time-varying covariates effects in multiplicative intensity models, Scandinavian Journal of Statistics 29: 57-74.

Marzec, L. \& Marzec, P. (1997). On fitting Cox's regression model with time-dependent coefficients, Biometrika 84: 901-908.

McKeague, I. W. (1988). A counting process approach to the regression analysis of grouped survival data, Stochastic Processes and their Applications 28: 221-239.

Murphy, S. A. (1993). Testing for a time dependent coefficient in Cox's regression model, Scandinavian Journal of Statistics 20: 35-50. 
Murphy, S. A. \& Sen, P. (1991). Time-dependent coefficient in a Cox-type regression model, Stochastic Processes and their Applications 39: 153-180.

Pedroso de Lima, A. C. \& Sen, P. K. (1999). Time-dependent coefficient in a multi-event model for survival analysis, Journal of Statistical Planning and Inference 75: 393-414.

Pérez-Ocón, R., Ruiz-Castro, J. E. \& Gámiz-Pérez, M. L. (2001). Non-homogeneous Markov models in the analysis of survival after breast cancer, Journal of the Royal Statistical Society, Series C 50: 111-124.

Restiffe, A. P. (2007). O desenvolvimento motor de recém-nascidos pré-termo e a termo até a aquisição da marcha segundo Alberta Infant Motor Scale: um estudo de coorte, Tese de Doutorado, FM/USP, São Paulo.

Scheike, T. \& Zhang, M.-J. (2007). Direct modelling of regression effects for transition probabilities in multistate models, Scandinavian Journal of Statistics 34: 17-32.

Shoenfeld, D. (1980). Chi-squared goodness-of-fit tests for the proportional hazards regression model, Biometrika 67: 145-153.

Tunes da Silva, G. (2005). Estimação da esperança do tempo de sobrevivência ajustado pela qualidade de vida por meio da modelagem de tempos de permanência, Tese de Doutorado, IME/USP, São Paulo.

Zucker, D. M. \& Karr, A. F. (1990). Nonparametric survival analysis with time-dependent covariate effects: a penalized partial likelihood approach, The Annals of Statistics 18: 329353. 